NBSIR $86-3340$

\title{
Effects of Global Density and Reynolds Number Variations on Mixing in Turbulent, Axisymmetric Jets
}

William M. Pitts

U.S. DEPARTMENT OE COMMERCE

National 3ureau of Standards

National Engineering Laboratory

Center for Fire Research

Gaithersburg, MD 20899

Niarch 1986

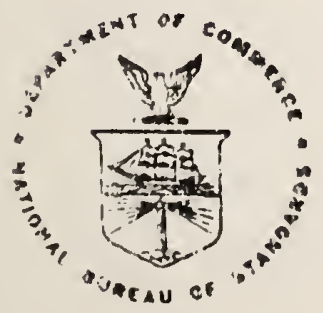

U.S. DEPAATINENT OF CCMAMEMCE

". itional bureal of giandakds.

QC

100

.1056

N0.86-3340 

NBSIR $86-3340$

EFFECTS OF GLOBAL DENSITY AND

REYNOLDS NUMBER VARIATIONS ON

MIXING IN TURBULENT, AXISYMMETRIC

JETS

William M. Pitts

U.S. DEPARTMENT OF COMMERCE

National Bureau of Standards

National Engineering Laboratory

Center for Fire Research

Gaithersburg, MD 20899

March 1986

U.S. DEPARTMENT OF COMMERCE, Maicolm Baldrige, Secretary NATIONAL BUREAU OF STANDARDS. Ernest Ambler, Director 

LIS T OF TABLES $\ldots \ldots \ldots \ldots \ldots \ldots \ldots \ldots \ldots \ldots \ldots \ldots \ldots \ldots \ldots \ldots \ldots \ldots \ldots \ldots \ldots$

LIST OF FIGURES $\ldots \ldots \ldots \ldots \ldots \ldots \ldots \ldots \ldots \ldots \ldots \ldots \ldots \ldots \ldots \ldots \ldots \ldots \ldots \ldots \ldots \ldots$

Abstract $\ldots \ldots \ldots \ldots \ldots \ldots \ldots \ldots \ldots \ldots \ldots \ldots \ldots \ldots \ldots \ldots \ldots \ldots \ldots \ldots \ldots \ldots \ldots$

1. INTRODUCTION $\ldots \ldots \ldots \ldots \ldots \ldots \ldots \ldots \ldots \ldots \ldots \ldots \ldots \ldots \ldots \ldots \ldots \ldots \ldots \ldots \ldots \ldots \ldots \ldots . . \ldots$

2. REVIEW OF AXISYMMETRIC JETS $\ldots \ldots \ldots \ldots \ldots \ldots \ldots \ldots \ldots \ldots \ldots \ldots \ldots \ldots \ldots \ldots$

2.1 Constant Density Axisymmetric Turbulent Free Jets .......... 4

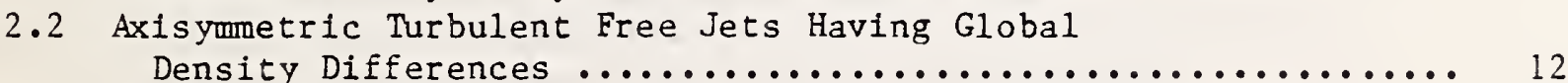

2.3 Effects of Buoyancy and Coflowing Surrounding Gases on Mixing in Axisymmetric Turbulent Jets ............... 25

3. EXPERIMENTAL SYSTEMS AND DATA TREATMENT ..................... 34

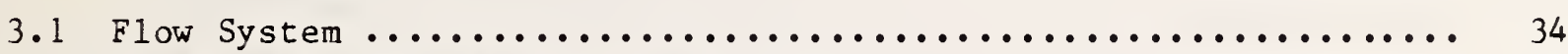

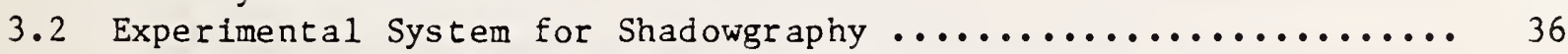

3.3 System for Rayleigh Light Scattering Measurements of

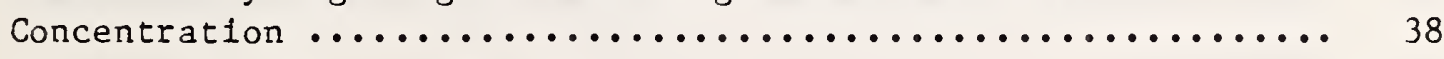

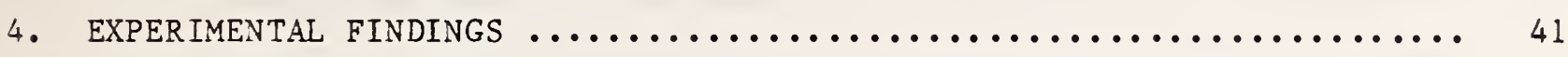

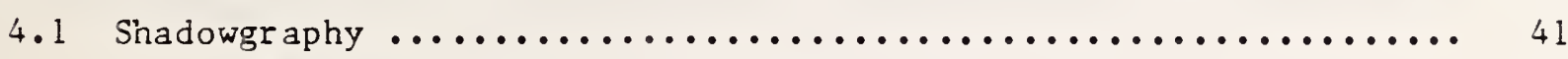

4.1 .1 Helium Turbulent Jet into Air $\left(R_{0}=0.14\right) \ldots \ldots \ldots \ldots \ldots .41$

4.1.2 Methane Turbulent Jet into Air $\left(R_{\rho}=0.55\right) \ldots \ldots \ldots \ldots . .43$

4.1.3 Propane Turbulent Jet into Carbon Dioxide $\left(R_{0}=1.02\right) \ldots 43$

4.1.4 Propane Turbulent Jet into Air $\left(R_{\rho}=1.55\right) \ldots \ldots \ldots \ldots \ldots .44$

4.1.5 Sulfur Hexafluoride Turbulent Jet into Air $\left(R_{\rho}=5.11\right) \ldots \quad 45$

4.1.6 Sulfur Hexafluoride Turbulent Jet into

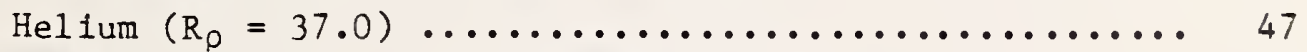

4.1.7 Summary of Trends in Mixing Behavior with

Changes in $R_{\rho}$ and $R e$ Derived from Shadowgraphs ...... 47

4.2 Concentration Measurements Along the Centerlines of

Variable Density Jets ............................ 49

4.2.1 $R_{\rho}$ Dependence of Canterline Average Concentration ...... 51

4.2.2 Unmixedness Measurements Along the Centerlines of Variable Density Jets ....................... 53

4.2.3 Density Effects on Skewness and Kurtosis Values Along

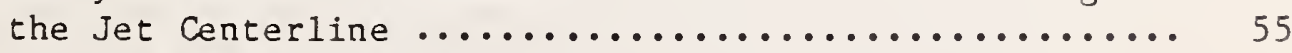

4.2.4 Reynolds Number Effects on Centerline Concentration

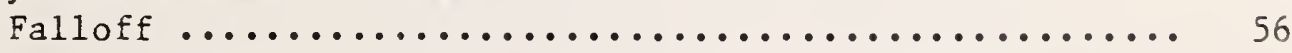

4.2.5 Reynolds Number Effects on Centerline Unmixedness

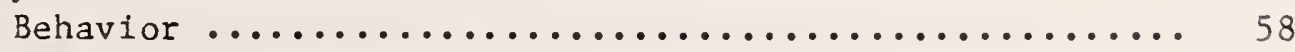

4.2.6 Reynolds Number Effects on Centerline Skewness and Kurtosis Values 
TABLE OF CONTENTS (continued)

$\underline{\text { Page }}$

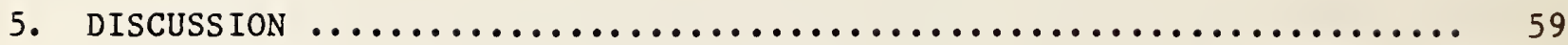

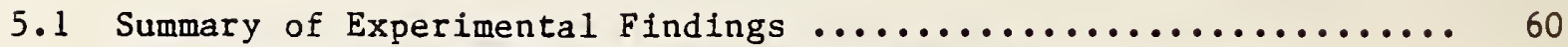

5.1.1 Effects of Global Density Differences on Turbulent

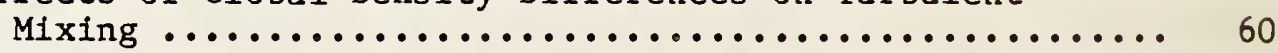

5.1.2 Effects of Reynolds Number Variations on Turbulent

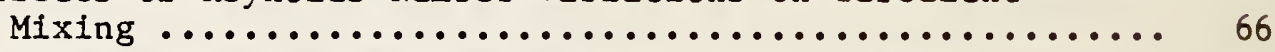

5.2 Comparison of Average Centerline Behavior with Integral

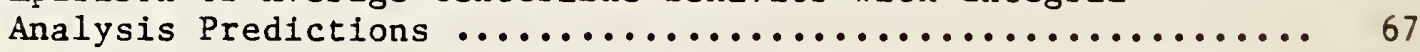

5.3 Comparison of Experimental Results with Past Work .......... 71

5.3.1 Average Centerline Concentration Measurements ......... 72

5.3.2 Unmixedness Centerline Measurements ................ 75

5.3.3 Centerline Measurements of Skewness and Kurtosis ...... 82

5.4 Centerline Falloff of Average Concentration and Growth of Unmixedness--Theortical Considerations ................. 83

5.5 Validity of Generalizing Results of This Study to Momentum-Driven Free Jets ....................... 90

6. FINAL REMARKS $\ldots \ldots \ldots \ldots \ldots \ldots \ldots \ldots \ldots \ldots \ldots \ldots \ldots \ldots \ldots \ldots \ldots \ldots \ldots \ldots \ldots . \ldots \ldots$

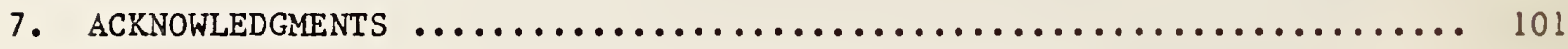

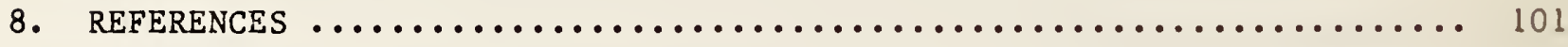

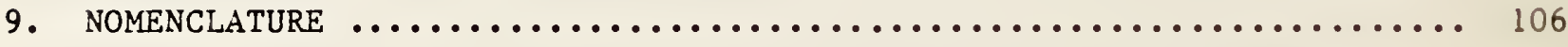




\section{LIST OF TABLES}

$\underline{\text { Page }}$

Table 1. Suppliers and Purities of Gases Used in This Work ......... 110

Table 2. Properties of Flows Studied .......................... 111

Table 3. Estinated Values from Shadowgraphs ..................... 112

Table 4. Predicted and Observed Rayleigh Scattering Cross Section Ratios .................................... 113

Table 5. Results for Average Concentration Measurements ........... 114

Table 6. $\mathrm{K}_{\mathrm{c}}$ Values which Result when Exponent in Equation (22) is

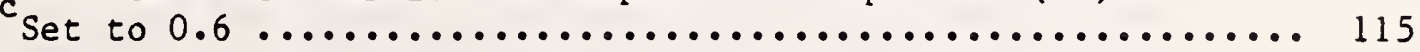

Table 7. Values of $z_{\max }$ Calculated Using Equations (58) and (59) ..... 116

Table 8. Summary of Average Centerline Concentration Measurements for Axi symmetric Turbulent Jets ..................... 117

Table 9. Literature Measurements of Centerline Unmixedness in Axisymmetric Turbulent Jets ..................... 118

Table 10. Centerline Unmixedness Results for this Study ............ 119 
Figure 1. A schematic representation of the three flow regions of an axisymmetric, constant-density, turbulent jet is shown. Representative radial profiles of velocity and jet fluid concentration are shown at one location in the potential core portion of the flow and at three positions in the self-similar regime

Figure 2. Calculated values for the ratio of the integrals $\mathrm{I}_{3}$ (eq. (44)) and $I_{2}$ (eq. (43)) are plotted as a function of

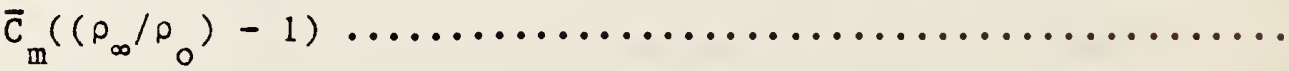

Figure 3. The experimental arrangement employed for time-resolved shadowgraphy of variable density flows is shown ...........

Figure 4. This figure shows the experimental system for making real-time, spatially-resolved, concentration measurements in turbulent flows of binary gas mixtures $\ldots \ldots \ldots \ldots \ldots \ldots \ldots$

Figure 5. Three shadowgraphs of a turbulent helium jet flowing into a slow coflow of air are superimposed. The Reynolds number

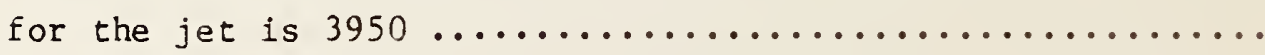

Figure 6. Superimposed shadowgraphs of the same helium jet visualized in fig. 5 are shown, but in this case, there is no coflow and the surroundings are the ambient laboratory a1r ........

Figure 7. Four superimposed shadowgraphs are reproduced for a turbulent methane jet at a Reynolds number of 3950

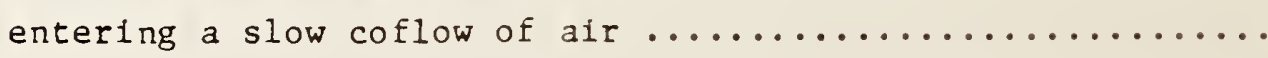

Figure 8. The superposition of four shadowgraphs is shown for a turbulent jet of propane flowing into a slow coflow of carbon dioxide. The Reynolds number for the jet 1 s $3960 \ldots$ 


\section{LIST OF FIGURES (continued)}

$\underline{\text { Page }}$

Figure 9. Shadowgraphs of the same propane jet visualized in fig. 8, but flowing into a slow coflow of air, are shown ....

Figure 10. The Reynolds number of the propane jet shown in fig. 9 has been increased to 7930 for the four shadowgraphs which have been superimposed. The coflow gas is again air .......

Figure 11. Four superimposed shadowgraphs are shown for a turbulent jet of propane $(\operatorname{Re}=11,880)$ entering a slow

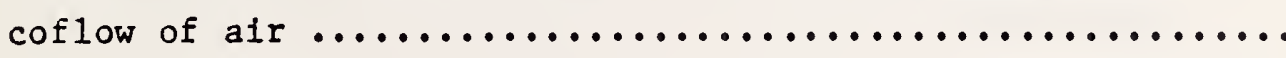

Figure 12. The near-field region of a sulfur hexafluoride turbulent jet $(\operatorname{Re}=3950)$ is visualized by shadowgraphy. Note the presence of jet fluid in regions well outside of the jet .... 131

Figure 13. A shadowgraph for the same flow described in fig. 12 is shown at larger downstream distances. Again, jet gas is observed in locations well outside of the jet ........... 132

Figure 14. The formation of a fountain by the strongly negatively buoyant jet of $\mathrm{SF}_{6}(\mathrm{Re}=3950)$ flowing into a coflow of air is visualized by shadowgraphy. The range of downstream

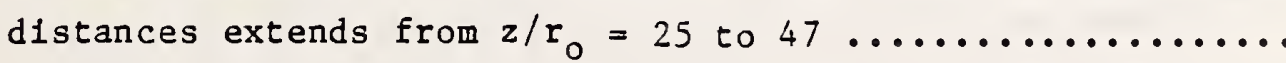

Figure 15. Four superimposed shadowgraphs of a turbulent jet of sulfur hexafluoride $(\operatorname{Re}=7890$ ) entering a slow coflow

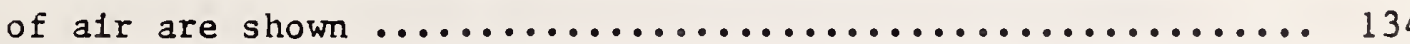

Figure 16. The Reynolds number has been increased to 11,860 for the sulfur hexafluoride turbulent jet entering a slow coflow

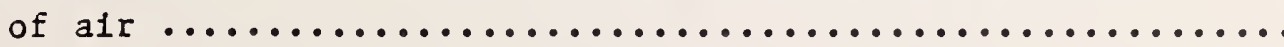




\section{LIST OF FIGURES (continued)}

$\underline{\text { Page }}$

Figure 17. The near field region of a $\mathrm{SF}_{6}$ jet ( $\operatorname{Re}=3950$ ) flowing into a slow coflow of helium is visualized by shadowgraphy .. 136

Figure 18. A shadowgraph centered at $z / \mathrm{r}_{0} \approx 24$ is shown for the $\mathrm{SF}_{6}$ jet of $\operatorname{Re}=3950$ flowing into a slow coflow of helium ..... 137

Figure 19. The fountain formed by the $\mathrm{SF}_{6}$ jet ( $\mathrm{Re}=3950$ ) entering into helium can be clearly seen in this shadowgraph recorded for downstream distances extending from $z / r_{0}=25$ to 47

Figure 20. A portion of the time-resolved methane concentration fluctuations within a turbulent jet of methane $(\operatorname{Re}=3950)$ flowing into a slow coflow of air is shown. The measurements were made on the centerline at $z / r_{0}=31.5 \ldots \ldots \ldots \ldots$

Figure 21. Values of $\mathrm{Y}_{\mathrm{o}} / \overline{\mathrm{Y}}_{\mathrm{m}}$ as functions of $\mathrm{z} / \mathrm{r}_{\mathrm{o}}$ are shown for the $\mathrm{SF}_{6}$ jet $(\mathrm{Re}=3950)$ flowing into coflows of air and hellum $\ldots 140$

Figure 22. Inverse centerline values of jet gas mole fraction, normalized by multiplying by the jet gas mole fraction $\left(\mathrm{X}_{\mathrm{O}}=1\right)$, are plotted as functions of nondimensionalized downstream distance for six different jet/coflow gas pair combinations. Straight lines are linear least squares

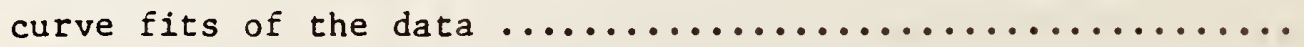

Figure 23. Values of the normalized centerline inverse mass fraction $\left(Y_{0} / \bar{Y}_{m}\right)$ are plotted as functions of $z / r_{0}$ for the six jet/ coflow pairs listed. Straight lines are linear least squares

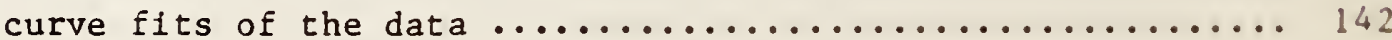




\section{LIST OF FIGURES (continued)}

Page

Figure 24. Values of $Y_{0} / \bar{Y}_{m}$ versus $z / r_{0}$ are plotted for the helium jet $(\operatorname{Re}=3950)$ flowing into both a slow coflow of air and the laboratory. Lines are linear least squares curve fits to the data .......................... 143

Figure 25. Experimental values of $Y_{o} / \bar{Y}_{m}$ for the six jet/coflow combinations listed are plotted as functions of downstream distance normalized by the effective radius, ${ }^{r} \varepsilon$. Results have been offset by the appropriate values of virtual origin. Comparison with fig. 23 indicates that the use of the effective radius concept gives a partial correlation of the data .......................... 144

Figure 26. Experimental results of centerline unmixedness measurements in mole fraction terms $\left(X_{m}^{\prime} / \bar{X}_{m}\right)$ are plotted as functions of $z / r_{0}$ for the $s i x j e t / c o f l o w$ palrs listed $\ldots \ldots \ldots \ldots \ldots \ldots \ldots$

F1gure 27. The results of $\mathrm{Y}_{\mathrm{m}}^{\prime} / \overline{\mathrm{Y}}_{\mathrm{m}}$ measurements for the jet/coflow pairs listed are plotted as functions of $z / r_{0}$. Note that the values for each flow approach a common asymptote of

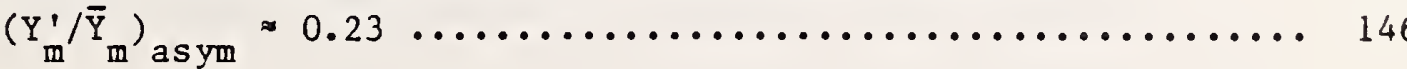

Figure 28. Values of $\mathrm{Y}_{\mathrm{m}}^{\prime} / \bar{Y}_{\mathrm{m}}$ are plotted as functions of $z / \mathrm{r}_{\mathrm{o}}$ for the helium jet ( $\operatorname{Re}=3950)$ flowing into a coflow of air and into

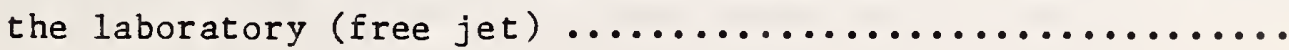

Figure 29. Mass fraction unmixedness values for the six jet/coflow gas combinations are plotted as functions of the downstream distance nondimensionalized by dividing by the effective radius. An excellent correlation of the data results ...... 


\section{LIST OF FIGURES (continued)}

Page

Figure 30. Skewness values for centerline concentration fluctuations are plotted as functions of $z / r_{0}$ for the jet/coflow gas

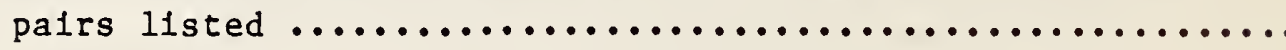

Figure 31. Kurtosis values for centerline concentration fluctuations are plotted as functions of $z / r_{0}$ for the jet/coflow gas

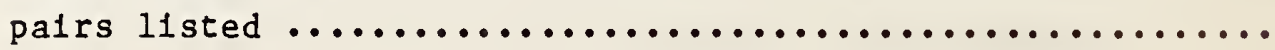

Figure 32. $Y_{0} / \bar{Y}_{m}$ values are plotted against $z / r_{0}$ for propane jets having the three different Reynolds numbers listed. The coflow gas is air. Stralght lines represent linear least

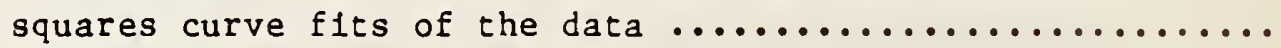

Figure 33. $Y_{0} / \bar{Y}_{m}$ values for two different Re flows of carbon tetrafluoride into air coflows are plotted as functions of $z / r_{0}$. Linear least squares curve fits to the data are

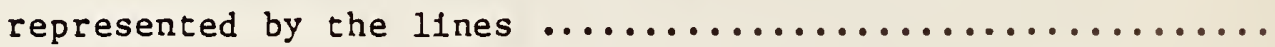

Figure 34. Values of $\mathrm{Y}_{\mathrm{o}} / \overline{\mathrm{Y}}_{\mathrm{m}}$ for $\mathrm{SF}_{6}$ jets at Reynolds numbers of 7890 and 11,860 are shown as functions of $z / r_{0}$. Stralght 11 nes represent the results of linear least

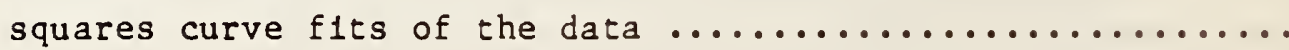

Figure 35. Virtual origins which are obtalned from centerline concentration measurements in the $\mathrm{C}_{3} \mathrm{H}_{8} / \mathrm{alr}$ and $\mathrm{CF}_{4} / \mathrm{a} 1 \mathrm{r}$ flows are plotted as functions of jet Reynolds numbers .....

F1gure 36. Mass fraction unmixedness values are plotted as functions of $z / r_{0}$ for three different Re jets of propane entering

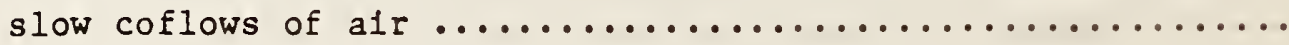


Figure 37. Mass fraction unmixedness values are plotted as functions of $z / r_{0}$ for two different Re jets of $\mathrm{CF}_{4}$ flowing into

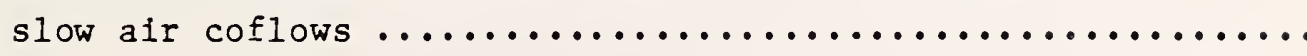

Figure 38. Unmixedness values for jets of $S F_{6}$ at Reynolds numbers of 7890 and 11,860 are plotted as functions of $z / r_{0}$. The coflow gas is air $\ldots \ldots \ldots \ldots \ldots \ldots \ldots \ldots \ldots \ldots \ldots \ldots \ldots \ldots$

Figure 39. Values of $1 / \vec{C}_{m}$ calculated using eq. (34) are plotted as functions of $z / r_{0}$ for the six gas pairs listed. These results are based on a method suggested by Thring and

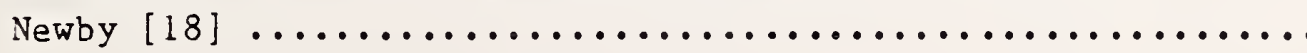

Figure 40. Values of $\bar{C}_{m}$ calculated in the manner suggested by Rod1 [5,23] are plotted as functions of $z / r_{0}$. Values of jet to surrounding gas density ratios correspond to those used in this study $\ldots \ldots \ldots \ldots \ldots \ldots \ldots \ldots \ldots \ldots \ldots \ldots \ldots \ldots \ldots \ldots \ldots$

Figure 41. The calculations shown in fig. 40 have been redrawn as $1 / \bar{C}_{\mathrm{m}}$ versus $z / r_{0}$. Comparison of this figure with fig. 39 shows that the approximate treatments of Thring and Newby [18] and Rodi [5,23] give similar predictions for the dependence of average centerline concentration falloff on the jet/coflow gas density ratio 

EFFECTS OF GLOBAL DENSITY AND REYNOLDS NUMBER VARIATIONS

ON MIXING IN TURBULENT, AXISYMMETRIC JETS

\author{
William M. Pitts \\ Center for Fire Research \\ National Bureau of Standards \\ Gaithers burg, MD 20899
}

\title{
Abstract
}

Shadowgraphy and laser-induced Rayleigh light scattering measurements of centerline concentration have been utilized to investigate the effects of global density and Reynolds number variations on the mixing behavior of a turbulent, axisymmetric jet. Density variations are introduced by using different gases for the jet and the surrounding coflow. Values of the jet/coflow density ratio have been varied from 0.14 to 37 . The shadowgraph measurements give a qualitative indication of the variations in average mixing behavior and turbulent structure which occur when the jet/coflow density ratio and the $\operatorname{Re}$ are varied. These trends are quantified by the Rayleigh scattering concentration measurements which give measurements of average centerline concentration as well as the second through fourth moments of the concentration fluctuations. Extensive comparisons of experimental results are made with previous literature findings. Integral analysis has been employed to provide a framework for analysis of the data. The results of this study have led to the proposal of a simple, qualitative theory based on two reasonable assumptions concerning the flow behavior which gives predictions which are in agreement with the experimental findings. 


\section{INTRODUCTION}

There has been a great deal of recent interest in improving the understanding of chemically reacting turbulent flow. The impetus for this research has been the realization that the complicated coupling of heat release and aerodynamics which occurs in such flows is crucial to control and improvement in such important areas as combustion and engine efficiencies, commercial chemical reactors, and the formation of pollutants in these systems.

With these considerations in mind, a long term experimental investigation of chemically reacting turbulent flow has been initiated which has as its primary goal an improved understanding of this important process. It is anticipated that the experimental findings will yield an improved physical understanding of turbulent flow behavior and at the same time provide the input for workers who are trying to model this complicated process. As a first step toward these goals, turbulent flows of two gases are being invest1gated in which there are global density differences based on molecular weight. This is appropriate, since the major coupling of chemical reactions and aerodynamics in a turbulent flow occurs through the localized density fluctuations introduced by chemical heat release. Despite the importance of density variations to understanding chemically reacting turbulent flow, the effects of global density differences remain poorly characterized.

In this work the mixing behavior of an axisymmetric turbulent jet with a slow coflow of a second gas has been considered. Gas pairs having jet/coflow density ratios from 0.14 to 37 were investigated. Flow visualization us 1 ng shadowgraphy was employed to obtain an overall picture of the effects of 
global density differences on turbulent mixing. These effects were quantified by performing a series of measurements of jet gas concentration along the centerlines of the axisymmetric jets. Laser-induced Rayleigh light scattering [1-4] was employed as the real-time, spatially-resolved concentration probe for these binary gas mixtures.

The following section (2) summarizes past experimental work on mixing in constant and variable density axisymetric jets and discusses integral analysis approaches to the solution of the equations for momentum and jet fluid conservation which have been used to gain insight into the time-averaged behavior of these flows. This is followed by a description of some considerations of special importance to this study. In particular, the effects of the presence of a coflow within an enclosure and the effects of buoyancy are emphasized. Section 3 describes the experimental systems for shadowgraphy and laser-induced Rayleigh light scattering. The experimental findings are summarized in section 4 and a discussion of the findings and comparison with past research is included in section 5. A simple theoretical framework based on the experimental results is proposed which is in qualitative agreement with the experimental findings. The final section (6) summarizes the major conclusions of this work.

\section{REVIEW OF AXISYMMETRIC JETS}

Axisymmetric turbulent jets have been the subject of intense investigation (both theoretical and experimental) for several decades. Such flows are of interest for several reasons. Among these are: (1) the axisymmetric symmetry simplifies some analyses, (2) these flows are easily generated in a laboratory environment, (3) many practical systems employ such flows, and

(4) these flows can serve as simple analogs for more complicated flows. 
The primary emphasis of this work is the effects of global density differences on turbulent mixing. However, it is important to review some general findings concerning the behavior of axisymmetric turbulent jets in order to develop a foundation and consistent body of nomenclature for the detailed discussion which follows. In the next subsection some general findings of experimental studies as well as predictions and conclusions from simple integral treatments of the equations of mass conservation and motion for isothermal, constant-density, free jets (sometimes called submerged turbulent jets) are discussed. This is followed by a subsection describing similar findings for variable density jets. Again, integral analysis of the equations of motion and mass conservation are utilized to give insight into the mixing behavior of these flows. The final subsection discusses specific aspects of the current study which might be expected to modify the turbulent mixing from that found for a purely momentum-driven axisymmetric free jet. In particular, modification of flow behavior due to the presence of a surrounding coflow and buoyancy effects are emphasized.

\subsection{Constant Density Axisymmetric Turbulent Free Jets}

There is a great deal of information available concerning the behavior of this type of turbulent flow. Figure 1 shows an idealized picture of the velocity and jet fluid concentration profiles of a constant density, axisymmetric, turbulent jet at high Reynolds number (Re). This figure represents a synopsis of results from many different studies. It shows the time-averaged behavior of the flow field which is found to maintain the axisymmetric symmetry imposed by the circular nozzle. 
The flow development of constant density jets can be broken into three distinct flow regimes as a function of downstream distance. After the jet gas exits the nozzle, a shear layer forms on the outer edge of the jet which grows both inwards toward the centerline of the flow as well as outwards into the surrounding gas as the flow moves downstream. There is a region in the center of the jet, called the potential core, where the velocity and jet fluid concentration have the same value as at $z=0$. As the jet fluid moves downstream, the radius of the potential core decreases until it disappears. The region of the flow field extending from the nozzle to the end of the potential core defines the first flow regime. Following the potential core region, the jet undergoes a development phase during which it assumes the velocity and concentration behavior characteristic of its final regime. For this reason, we will denote this second flow region as the development regime. The final regime is called the self-similarity regime. The characteristics of this flow region are discussed further below.

A flow field is said to be self-similar when only one geometrical variable is required to characterize the nondimensionalized, time-averaged behavior of the concentration or velocity throughout the region. In an analogous way, local simflarity is defined as occurring when concentration or velocity profiles can be collapsed in terms of a local geometrical variable. These definitions are the same as suggested by Chen and Rod1 [5].

The integral equations for momentum and jet fluid conservation can be used to derive the requirements necessary for an axisymetric, constant density, turbulent jet to have a self-similar behavior. These conservation equations can be written as [6], 


$$
2 \pi \rho \int_{0}^{\infty} U^{2} r d r=M_{0}
$$

and

$$
2 \pi \rho \int_{0}^{\infty} \bar{C} \bar{U} r d r=N_{0},
$$

where $M_{0}$ and $N_{0}$ are constants representing the momentum flux and jet fluid concentration flux, respectively. For now, the exact nature of $\bar{C}$ has not been specifled since this is not critical for constant density flows. In section 2.2 a more precise description of $\bar{C}$ will be given. If the flow fleld 1 s selfsimilar, the local values of $\bar{U}$ and $\bar{C}$ can be expressed as functions of the similarity parameter $n$ (where $n=r / z$ ) as,

$$
\overrightarrow{\mathrm{U}}=\overline{\mathrm{U}}_{\mathbf{m}} \mathrm{f}_{2}(n)
$$

and

$$
\bar{C}=\bar{C}_{m} f_{1}(n),
$$

where the subscript m indicates values on the fet centerline. The following equations are obtained by substituting eqs. (3) and (4) into eqs. (1) and (2) and assuming uniform exit profiles at the nozzle for both concentration and velocity,

$$
2 \pi \rho \vec{U}_{\mathrm{m}}^{2} z^{2} \int_{0}^{\infty} \mathrm{f}_{2}^{2} n d n=\pi \rho r_{0}^{2} U_{0}^{2}
$$

and 


$$
2 \pi \rho \bar{C}_{m} \bar{U}_{m} z^{2} \int_{0}^{\infty} f_{1} f_{2} n d n=\pi \rho r_{0}^{2} U_{0} C_{0} .
$$

The assumption of uniform jet exit distributions for concentration and velocity simplifies calculations, but is not necessary for the final conclusions.

Eq. (5) yields directly

$$
\frac{U_{0}}{\vec{U}_{m}}=\frac{z}{r_{0}} \sqrt{\mathrm{K}_{22}}
$$

where $\mathrm{K}_{22}$ is a constant defined as

$$
K_{22}=\int_{0}^{\infty} 2 f_{2}^{2} n d n
$$

Substituting eq. (7) into eq. (6) gives a similar equation for the centerline concentration,

$$
\frac{\mathrm{C}_{\mathrm{o}}}{\overline{\mathrm{C}}_{\mathrm{m}}}=\frac{\mathrm{z}}{\mathrm{r}_{\mathrm{o}}} \frac{\mathrm{K}_{12}}{\sqrt{\mathrm{K}_{22}}}
$$

with

$$
K_{12}=\int_{0}^{\infty} 2 f_{1} E_{2} n d n
$$

Equations (7) and (9) require that in the self-similarity region of the flow field that both jet fluid concentration and velocity have a hyperbolic dependence on downstream distance. Experimentally, it is often found that the origins of plots of $C_{0} / \bar{C}_{m}$ and $U_{0} / \bar{U}_{m}$ as functions of $z / r_{0}$ are not located at 
the jet exit and a virtual origin $\left(z_{0}\right)$ is introduced. In terms of $z_{0}$, eqs. (7) and (9) are rewritten as

$$
\frac{\mathrm{U}_{\mathrm{o}}}{\overline{\mathrm{U}}_{\mathrm{m}}}=\mathrm{K}_{\mathrm{u}} \frac{z-\left(\mathrm{z}_{\mathrm{o}}\right)_{\mathrm{u}}}{\mathrm{r}_{\mathrm{o}}}
$$

and

$$
\frac{\mathrm{c}_{\mathrm{o}}}{\overline{\mathrm{C}}_{\mathrm{m}}}=\mathrm{K}_{\mathrm{c}} \frac{z-\left(z_{\mathrm{o}}\right)_{\mathrm{c}}}{\mathrm{r}_{\mathrm{o}}}
$$

where $\mathrm{K}_{22}$ and $\mathrm{K}_{12} / \sqrt{\mathrm{K}_{22}}$ are replaced by $\mathrm{K}_{\mathrm{u}}$ and $\mathrm{K}_{c}$, respectively, to reflect the fact that these parameters are usually determined empirically from experiment instead of directly integrating the radial profiles to obtain values for $k_{22}$ and $\mathrm{K}_{12}$.

In general, experiments by a large number of workers have yielded results which are in agreement with the qualitative predictions based on the above discussion. Both average velocity and concentration centerline decays are found to have a hyperbolic falloff with downstream distance when measured from a sultably chosen virtual origin. Radial profiles of these parameters are found to collapse to single curves when plotted in terms of $\bar{U} / \bar{U}_{\mathrm{m}}$ or $\bar{C} / \bar{C}_{\mathrm{m}}$ as functions of $n$. In almost all cases the resulting dependence of velocity and concentration on $\eta$ has been found to be well fit by Gaussian curves. The halfwidth for the velocity (defined as the value of $n$ where $\bar{U} / \bar{U}_{m}=1 / 2$ ) is found to be less than the corresponding value for concentration. This finding Indicates that momentum is transferred less efficiently than mass. 
Unfortunately, quantitative agreement among different studies is not nearly as complete. Values of both $K_{u}$ and $K_{c}$ are found to vary widely from experiment to experiment. In a recent review, List [7] recommended a value of 0.081 for $K_{u}$ based on the results of several different studies. To give an idea of the variations which are observed, this value can be compared with that of $\mathrm{K}_{\mathrm{u}}=0.093$ reported by Wygnanski and Fiedler [8] in their widely quoted investigation. Becker et al [9] have listed values of $K_{c}$ measured by several different authors using efther temperature, concentration, or particle-marked constant-density flows. A range of values from 0.088 to 0.139 are tabulated. Chen and Rodi [5] recommend a value of $\mathrm{K}_{c}=0.10$ for the axisymmetric jet based on their review of literature results. These workers conclude that the observed "differences merely represent experimental scatter".

Some authors have concluded that variations observed in values of $\mathrm{K}_{\mathrm{u}}$, $\mathrm{K}_{\mathrm{c}}$, and $z_{0}$ are due to slow development of the jets to self-similar behavior. For instance, Wygnanski and Fiedler [8] found that self-similar behavior of their jet only occurred for values of $z / r_{0}>140$. Measurements of $\bar{U}_{m}$ made at considerably shorter downstream distances seemed to obey a self-similarity relationship, but linear fits to the data gave different values of $\mathrm{K}_{\mathrm{u}}$ and $\left(z_{0}\right)_{u}$ than found when data from further downstream were utilized. Birch et al [10] have reached similar conclusions concerning the approach to selfsimilarity for jet fluid concentration. However, it should be noted that this study was done for a flow of methane into air and density effects can not be ruled out. In contrast to the results discussed above, most workers have conciuded that self-similarity for this type of flow occurs within 20 to 40 radii of the nozzle exit. 
In quantifying centerline decays of these jets the position of the virtual origin may play a crucial role. Some workers utilize the value of $z_{0}$ as an experimental parameter while others have forced their fits of data to pass through $z=0$. Further complications arise due to the wide range of $z_{0}$ values which have been measured. Bradshaw [11] has argued that the position of the virtual origin depends on initial conditions at the jet exit. For instance, the nature of the boundary layer at the nozzle (laminar or turbulent) and the level of turbulence of the fluid inside the nozzle are both very important in determining the location of $z_{0}$.

Despite the complexity of the factors which are expected to determine the location of $z_{0}$, some information is available in the literature concerning its dependence on Re. In his critical evaluation of turbulent data, Harsha [12] notes a tendency for the virtual origin (he refers to it as the length of the potential core) to increase with increasing Re. Using data of Alexander et al [13], Harsha has derived an equation for $z_{0}$ which can be written

$$
\frac{\left(z_{0}\right)_{u}}{r_{0}}=4.3 \mathrm{Re}^{0.097}
$$

Ebrahimi [14] has reported similar shifts in the location of $\left(z_{1 / 2}\right)_{u}($ axial distance where $\bar{U}_{m}=U_{0} / 2$ ) with Re which obey the empirical relationship,

$$
\frac{\left(z_{1 / 2}\right) u}{r_{0}}=1.3 \times 10^{-4} \operatorname{Re}+23.2
$$

If one assumes the virtual origin shifts in a manner similar to $\left(z_{1 / 2}\right)_{u}$, this relationship indicates a downstream shift of the virtual origin with increasing Re. Ebrahimi and Kleine [15] found a similar dependence for the jet fluid concentration half length (value of $z$ where $\bar{C}_{m}=c_{0} / 2$ ), namely 


$$
\frac{\left(z_{1 / 2}\right)_{c}}{r_{0}}=2.6 \times 10^{-4} \mathrm{Re}+12
$$

This finding is evidence that the virtual origins for concentration also shift downstream with increasing Re.

Thus far, the discussion has centered on the behavior of average centerline values of velocity and concentration in axisymmetric jets. It is also necessary to consider the behavior of the turbulent fluctuations for these two parameters. Such fluctuation behavior is usually characterized by measuring average values of rms for the velocity $\left(U^{\prime}\right)$ and concentration ( $\left.C^{\prime}\right)$ fluctuations which occur in the flow field. Often such data are normalized by dividing by the average concentration or velocity at the measurement point. These latter measurements are known as fluctuation intensities. Often the concentration intensity is referred to as the unmixedness.

It is generally believed that both the concentration and velocity Intensities approach asymptotic values in the self-similar region of the flow field. In fact, the attainment of such asymptotic values is often taken as a criteria for self-similarity.

Very recently, Dahm [16] has reported that an axisymmetric turbulent jet of water does not appear to attain an asymtotic value of unmixedness even at extremely large downstream distances. This result has led this researcher to conclude that intensity is not a suitable criteria for self-similartty. A new similarity parameter defined as $\bar{C} /(1-\bar{C})$ has been proposed by this author to treat data of this type. 
Many measurements of $U_{m}^{\prime} / \bar{U}_{m}$ and $C_{m}^{\prime} / \vec{C}_{m}$ can be found in the literature. These measurements are characterized by rapid growth in values of the intensity as the measurement point is initially moved away from the nozzle. This is followed by a region at larger values of $z / r_{0}$ where the intensities appear to level off or rise very slowly. As is the case for the average values, quantitative agreement among different experiments which have measured velocity or concentration intensities is poor. The basis for such variations is not known at the present time.

\subsection{Axisymmetric Turbulent Free Jets Having Global Density Differences}

Historically, there has been a great deal of interest in variable density, axisymmetric, turbulent jets. The reviews by Harsha [12] and Chen and Rodi [5] discuss several of these studies. In section 5 the experimental findings of this study on mixing will be compared with many of these studies so a detailed description of individual studies will be delayed until then. Only a short discussion of general experimental findings for jets having global density differences is presented here along with some analyses of these flows using varlous integral approaches similar to those given above for the constant density case.

Only flows in which the global density differences are due to temperature or gas composition are considered. Constant pressure and incompressible behavior are assumed. For these conditions, global denstty differences due to temperature or composition are generally assumed to have equivalent effects on turbulent mixing. 
Several generalizations can be made concerning the behavior of these axisymmetric jets. Here focus is upon mixing behavior. As discussed below, analysis indicates that variable density jets can never attain true selfsimilarity. However, at far downstream distances where the centerline density approaches the ambient surrounding density, but for which the initial jet momentum forces still dominate, these jets will approach self-similarity.

Experimentally, it is found that such jets have hyperbolic centerline concentration dependencies on downstream distance, a characteristic of selfsimilar, constant-density jets. This is true even at locations where the centerline density is far from that of the ambient surroundings. Furthermore, it has been found that for moderate downstream distances where the jets do not display hyperbolic centerline concentration decay, local similarity still occurs. In particular, the radial profiles of average temperature or jet fluid concentration still have a Gaussian shape and such profiles, when taken at various downstream distances, collapse when values of $\bar{C} / \bar{C}_{m}$ are plotted as functions of $r /\left(r_{1 / 2}\right)_{c} \cdot\left(r_{1 / 2}\right)_{c}$ is the radius for which $\bar{c} / \bar{c}_{m}$ or $\left(\bar{T}-T_{\infty}\right) /\left(\bar{T}_{m}-T_{\infty}\right)=0.5$. A striking example of this behavior is found in the study of $0^{\prime}$ Connor et al [17]. These authors investigated the turbulent mixing of an axisymmetric jet of partially dissociated nitrogen with ambient air. Jet exit temperatures as high as $5700 \mathrm{~K}$ were utilized. For axial locations as near as $z / r_{0}=8$ from the nozzle, the radial profiles of mass fraction of jet gas were found to be locally similar and to obey a Gaussian falloff quite we 11.

All experimental results seem to agree that a decreasing density ratio for the jet to ambient surrounding gases $\left(R_{\rho}=\rho_{0} / \rho_{\infty}\right)$ leads to a faster fall- 
off of jet gas centerline concentration with downstream distance. Here "concentration" refers specifically to efther $\left(\bar{T}_{m}-T_{\infty}\right) /\left(T_{0}-T_{\infty}\right)$ in the case of heated or cooled flows or to the mass fraction of jet gas in cases where 1sothermal gas density differences are important. A justifi:ation for these forms of "concentration" w111 be glven shortly.

In his review, Harsha [12] concludes that the velocity potential core for varlable density jets lengthens as the ratio of jet to surrounding gas dens1ty is increased. In contrast to this result, he reports that the potential core for concentration (In terms of mole fraction) shortens with increasing $R_{\rho}$. This was considered to be an unexplained anomalous behavior. In section 5 it w111 become clear that this observation is an artifact due to the manner in wh1ch Harsha analyzed the data.

Several sets of experimental data for the decay of average centerline concentration with downstream distance are avallable in the literature for varlable-density, axisymmetric jets. Specific results are to be discussed in section 5. However, it is worthwhile to note that the quantitative results are scattered in the same way as those found for the constant density flows.

There have been relatively few measurements of turbulent fluctuation behavior in varlable density jets. At the present time, it seems falr to state that no consistent plcture has emerged and very little is understood concerning the predicted or experimental behavior of centerline velocity or concentration intensities in these flows. 
Some insight into the effects of global density differences on mixing behavior can be obtained by considering the integral equations for conservation of momentum and jet fluid. In cases where the density is not constant, eqs. (1) and (2) become

$$
2 \pi \int_{0}^{\infty} \overline{\rho U}{ }^{2} r d r=M_{0}
$$

and

$$
2 \pi \int_{0}^{\infty} \overline{\rho C \bar{U}} r d r=N_{0} .
$$

Since $\mathrm{M}_{0}$ and $\mathrm{N}_{0}$ are constants, the integrals on the left hand side of these equations must also be constant. However, the density terms inside the integrals are not constant. It is clear that there are no physically meaningful functions having the forms of eqs. (3) and (4) which can be independent of $n$. This conclusion requires that no variable density jet can have a truly self-similar behavior.

Despite the above conclusion, eqs. (16) and (17) can be used to make predictions concerning the behavior of certain types of flows having density variations. Thring and Newby [18] were apparently the first to creat these equations in such a manner. These workers first considered the case of a burning free jet for which it was assumed that the oniy effect of chemical heat release was to increase the temperature and hence decrease the density, and that in the region of interest the density was essentially uniform. Of course, the Re was assumed to be sufficient to insure that the flow was dominated by the initial jet momentum and that buoyancy effects could be 
neglected. These assumptions allowed the $\bar{p}$ term in eqs. (16) and (17) to be placed outside of the integrals. By assuming initially uniform jet profiles for velocity and jet fluid concentration, the equations can be rewritten as

$$
2 \pi \rho_{f} \int_{0}^{\infty} \bar{U}^{2} r d r=\pi r_{o}^{2} \rho_{o} U_{o}^{2}
$$

and

$$
2 \pi \rho_{f} \int_{0}^{\infty} \bar{C} \bar{U} r d r=\pi r_{o}^{2} \rho_{o} U_{0} C_{0}
$$

The values of the integrals in eqs. (18) and (19) should assume the same form as those for constant density flows. Making this assumption, the equations become

$$
\frac{U_{o}}{\bar{U}_{m}}=\frac{z}{r_{0}} \sqrt{\frac{\rho_{f}}{\rho_{0}}} \sqrt{K_{22}}
$$

and

$$
\frac{\mathrm{C}_{\mathrm{o}}}{\overline{\mathrm{C}}_{\mathrm{m}}}=\frac{z}{\mathrm{r}_{\mathrm{o}}} \frac{\mathrm{K}_{12}}{\sqrt{\mathrm{K}_{22}}} \sqrt{\rho_{\mathrm{f}} / \rho_{\mathrm{o}}}
$$

where $\rho_{0}$ is the density of the jet fluld at the exit, $\rho_{f}$ is the density at the measurement point, and $\mathrm{K}_{22}$ and $\mathrm{K}_{12}$ have the same forms as eqs. (8) and (10), respectively. Note that since the density is assumed to be constant, $\bar{C}_{m}$ can be either in terms of mass or mole units.

Thring and Newby [18] Incorporated the square root of the inftial to final jet density into an effective radius defined as 


$$
r_{\varepsilon}=r_{o} \sqrt{\rho_{o} / \rho_{f}}
$$

Physically, ${ }_{\varepsilon}$ corresponds to the radius that a nozzle would be required to have in order to result in a flow having the same momentum and mass flux as actually exists, but with a density $\rho_{f}$ instead of $\rho_{0}$.

By using this definition and making similar modifications to the formulas as made earlier for the constant density case, the expressions

$$
\frac{\mathrm{U}_{\mathrm{O}}}{\overline{\mathrm{U}}_{\mathrm{m}}}=\frac{z-\left(\mathrm{z}_{\mathrm{O}}\right)_{\mathrm{U}}}{\mathrm{r}_{\varepsilon}} \mathrm{K}_{\mathrm{u}}
$$

and

$$
\frac{\mathrm{C}_{\mathrm{o}}}{\overline{\mathrm{C}}_{\mathrm{m}}}=\frac{z-\left(\mathrm{z}_{\mathrm{O}}\right)_{\mathrm{c}}}{\mathrm{r}_{\varepsilon}} \mathrm{K}_{\mathrm{c}}
$$

are obtained.

These expressions indicate that for cases where the downstream density of a variable density axisymmetric jet can be assumed to be constant, the flow will behave in an analogous manner to a constant density flow if $r_{0}$ in eqs. (11) and (12) is replaced by $r_{\varepsilon}$. Since the centerline concentration of jet fluid approaches that of the surroundings as the downstream distance increases, it is clear that the behavior of a jet flowing into ambient surrounding gas at a different density will approach asymptotically the selfsimilar behavior indicated by eqs. (23) and (24) when $\rho_{f}$ in eq. (22) is replaced by the surrounding gas density, $\rho_{\infty}$. 
Thring and Newby [18] have also provided an approximate analysis of eqs. (16) and (17) which allows the behavior of centerline temperature to be predicted in cases where the difference between $\vec{\rho}_{m}$ and $\rho_{\infty}$ can not be considered as negligible. A similar analysis will be given here, but the derivation will be generalized to include cases where density differences are due to concentration or temperature. The following relationships for generalized concentration are obtained by assuming the ideal gas law holds everywhere In the flow field,

$$
\frac{\rho_{\infty}-\bar{\rho}}{\rho_{\infty}-\rho_{0}} \frac{\rho_{0}}{\bar{\rho}}=\frac{\bar{Y}}{\bar{Y}_{0}}=\frac{\bar{T}-\bar{T}_{\infty}}{\bar{T}_{0}-\bar{T}_{\infty}}=\bar{C}
$$

Substitution of this definition of $\bar{C}$ into eq. (17) gives an expression for the conservation of mass flow defect as orlginally defined by Corrsin and Uberol [6]. Note that eq. (25) can be rewritten as

$$
\vec{p}=\frac{\rho_{\infty}}{1+\bar{C}\left(\frac{\rho_{\infty}}{\rho_{0}}-1\right)}
$$

Substituting eqs. (3), (4), and (26) into eqs. (16) and (17) and assuming uniform Initial jet profiles yields the following equations for conservation of momentum,

$$
\frac{r_{0}^{2}}{z^{2}}=\frac{\rho_{\infty}}{\rho_{0}} \frac{\bar{U}_{m}^{2}}{U_{0}^{2}} \int_{0}^{\infty} \frac{2 f_{2}^{2} n d n}{1+\bar{C}_{m}\left(\frac{\rho_{\infty}}{\rho_{0}}-1\right) f_{1}}
$$

and jet fluid concentration,

$$
\frac{r_{0}^{2}}{z^{2}}=\frac{\rho_{\infty}}{\rho_{0}} \frac{\bar{U}_{m}}{U_{0}} \bar{C}_{m} \int_{0}^{\infty} \frac{2 f_{1} f_{2} n d n}{1+\bar{C}_{m}\left(\frac{\rho_{\infty}}{\rho_{0}}-1\right) f_{1}}
$$


Assuming $\overline{\mathrm{C}}_{\mathrm{m}} \ll 1$, $1 \mathrm{t}$ is possible to generate an approximate solution for $\bar{C}_{m}$ which is accurate to the order of $\bar{c}_{m}^{2}$. This is done by using the binomial expression to expand $1 /\left\{1+\bar{c}_{m}\left[\left(\rho_{\infty} / \rho_{0}\right)-1\right] f_{1}\right\}$ and neglecting terms in $\vec{C}_{m}$ of higher order than one. Using this procedure, eqs. (27) and (28) become

$$
\frac{r_{\varepsilon}^{2}}{z^{2}}=\frac{\bar{U}_{m}^{2}}{U_{0}^{2}}\left[k_{22}-\bar{C}_{m}\left(\frac{\rho_{\infty}}{\rho_{0}}-1\right) k_{122}\right]
$$

and

$$
\frac{r_{\varepsilon}^{2}}{z^{2}}=\frac{\bar{U}_{m}}{U_{0}} \bar{C}_{m}\left[K_{12}-\bar{C}_{m}\left(\frac{\rho_{\infty}}{\rho_{0}}-1\right) K_{112}\right]
$$

where $K_{22}$ and $K_{12}$ are given by eqs. ( 8 ) and (10), respectively. $K_{122}$ and $K_{112}$ are defined analogously as

$$
k_{122}=\int_{0}^{\infty} 2 f_{1} f_{2}^{2} n d n
$$

and

$$
k_{112}=\int_{0}^{\infty} 2 f_{1}^{2} f_{2} n d \eta
$$

Dividing eq. (30) by the square root of eq. (29), again using a series expansion, and neglecting higher order terms results in the following expression,

$$
\frac{\mathrm{r}_{\varepsilon}}{z}=\overline{\mathrm{C}}_{\mathrm{m}} \frac{\mathrm{K}_{12}}{\sqrt{\mathrm{K}_{22}}}\left[1+\overline{\mathrm{C}}_{\mathrm{m}}\left(\frac{\rho_{\infty}}{\rho_{\mathrm{o}}}-1\right)\left(\frac{\mathrm{K}_{122}}{2 \mathrm{~K}_{22}}-\frac{\mathrm{K}_{112}}{\mathrm{~K}_{12}}\right)\right]
$$


Equation (33) can be rearranged and solved for $1 / \bar{C}_{m}$. Again, series expansions are utilized and higher order terms are omitted. The final result is

$$
\frac{1}{\bar{C}_{m}}=\frac{K_{12}}{\sqrt{K_{22}}} \frac{z}{r_{\varepsilon}}+\left(\frac{\rho_{\infty}}{\rho_{0}}-1\right)\left[\frac{K_{122}}{2 K_{22}}-\frac{K_{112}}{K_{12}}\right]
$$

For small $z$, the term in brackets is expected to vary with $z$. At larger $z$ this term should become nearly constant. Thring and Newby [18] noted that the value of $\mathrm{K}_{122} / \mathrm{K}_{22}$ should be nearly equal to $\mathrm{K}_{122} / \mathrm{K}_{12}$ and hence the term in brackets is negative. This implies that the location of the virtual origin for experimental data plotted as $1 / \bar{C}_{m}$ versus $z / r_{\varepsilon}$ will be dependent on the density ratio $\rho_{0} / \rho_{\infty}$, with $\left(z_{0}\right)_{c}$ (as defined by eq. (24)) becoming negative for $R_{\rho}>1$ (negatively buoyant jet) and positive for $R_{\rho}<1$ (positively buoyant jet). Note also that the slope of the plot is predicted to be independent of $R_{\rho}$ and to be the same as that given in eq. (21).

There are several studies in the literature [e.g., 19, 20], mostly for heatud flows, which agree well with the predictions of Thring and Newby [18] with respect to variations in the slopes of $1 / \bar{C}_{\text {m }}$ versus $z / r_{0}$ plots with density ratio. There appears to have been no verification of their predictions for changes in $\left(z_{0}\right)_{c}$ with the same parameter.

Ricou and Spalding [21] have described a study which also supports the concept of the effective radius. These authors have experimentally determined the entrainment behaviors of variable density, axisymmetric, turbulent jets. For the constant density case, dimensional analysis can be used to show that the average mass flow rate $(\dot{m})$ through a cross section of the jet, deftned as 


$$
\dot{\mathrm{m}}=2 \pi \int_{0}^{\infty} \overline{\mathrm{\rho U}} \mathrm{r} \mathrm{d} r
$$

can be expressed in terms of other flow parameters as

$$
\frac{\dot{\mathrm{m}}}{z \mathrm{M}_{\mathrm{O}}^{1 / 2} \rho_{\infty}^{1 / 2}}=\mathrm{K}_{\mathrm{e}}
$$

where $\mathrm{K}_{e}$ is a constant to be determined experimentally.

The form of eq. (36) was verified for an air jet flowing into surrounding air. Interestingly, the same equation was found to be valid for jet flows of $\mathrm{CO}_{2}, \mathrm{C}_{3} \mathrm{H}_{8}$, and $\mathrm{H}_{2}$ into air indicating the result is independent of density. A value of $\mathrm{K}_{\mathrm{e}}=0.282$ was determined.

By assuming that the jet profiles at the nozzle are uniform, it is possible to rewrite eq. (36) as

$$
\frac{\dot{\underline{m}}}{\dot{\mathrm{m}}_{0}}=0.16 \frac{z}{\mathrm{r}_{0}}\left(\frac{\rho_{\infty}}{\rho_{0}}\right)^{1 / 2}=0.16 \frac{z}{r_{\varepsilon}}
$$

This equation agrees with the hypothesis of Thring and Newby [18] that the characteristic length for variable density jets is not $r_{0}$, but rather $r_{\varepsilon}$.

Equation (37) was found to be valid for $\operatorname{Re}>2.5 \times 10^{4}$ by Ricou and Spalding [21]. Measurements of the entrainment taken at $z / r_{0}=27.4$ indicated that for lower Re the amount of entrained fluid increased as the Re was decreased. The value of $\mathrm{K}_{e}$ and its independence of $\mathrm{Re}$ in the range 6-29 $\times 10^{4}$ was verified in an independent study by Hill [22]. This study differed from that of Ricou and Spalding in that the local entrainment coefficient was 
measured at a given downstream location instead of the integrated entralnment to that point. Hill's study showed that there 18 an initial region of the fet near the nozzle where the entrainment coefficlent is very small, and that it rapidly increases with downstream distance until a constant value 18 reached for $z / r_{0}>20$.

The experimental observations that eq. (36) can be used to describe fet mass flow rate through a cross section and that radial profiles of concentration and velocity obey local simflarity even when the flow is not selfsimflar, can be utilized to predict the behavior of the centerline concentration falloff for variable density flows. The only additional assumption which is required is that the turbulent Schmidt number $\left(\left(S_{C}\right)_{t u}\right)$ be a constant. This development should be valld for downstream locations where the above cond1tions are fulfilled, even if self-similarity behavior has not been approached. The findings of such a study have been summarized by Chen and Rodi [5]. The following analysis of this problem is based on the work of Rod1 (23).

Expressions similar to eqs. (3) and (4) can be written in terms of a local simflarity parameter to describe the radial dependence of concentration and velocity for a given value of $z / r_{0}$. These are

$$
\bar{C}=\bar{C}_{m} f_{l}^{\prime}\left(\eta^{\prime}\right)
$$

and

$$
\bar{U}=\bar{U}_{m} E_{2}^{\prime}\left(\eta^{\prime}\right),
$$


where the primes indicate expressions written in terms of the local similarity parameter, $\eta^{\prime}=r /\left(r_{1 / 2}\right)_{u}$.

Utilizing the experimental observation that the radial fallorfs of $\bar{C}$ and $\bar{U}$ have Gaussian forms and assuming a constant value for $(S c)_{t u}, f_{1}^{\prime}$ and $f_{2}^{\prime}$ can be written as

$$
f_{i}\left(n^{\prime}\right)=e^{\frac{-0.693}{R^{2}} n^{\prime 2}}
$$

and

$$
f_{2}^{\prime}\left(\pi^{\prime}\right)=e^{-0.693 \pi^{\prime 2}}
$$

where $\left(r_{1 / 2}\right)_{c}=R\left(r_{1 / 2}\right)_{U}$. With $R$ prescribed, there are three unknowns, namely $\bar{C}_{m}, \bar{U}_{m}$, and $\left(r_{1 / 2}\right)_{u}$ in eqs. $(38-41)$.

Three Independent equations are required to solve for these three unknowns. Rodi [5, 23] utilized the integral momentum equation (eq. (16)), the weight deficit equation (eq. (17) with $\bar{C}$ defined as in eq. (25)), and the entrainment law of Rlcou and Spalding [21] (eq. (37)). By making substitutions similar to those used above, these three equations can be written as

$$
\begin{aligned}
& 2 \pi \rho_{\infty}\left(r_{1 / 2}\right)_{u}^{2} \bar{U}_{m}^{2} \int_{0}^{\infty} \frac{f_{2}^{\prime 2} \eta^{\prime} d n^{\prime}}{1+\bar{C}_{m}\left(\frac{\rho_{\infty}}{\partial_{0}}-1\right) \bar{y}_{1}^{\prime}}=M_{0} \\
& 2 \pi 0_{\infty}\left(r_{1 / 2}\right)_{u}^{2} \bar{U}_{m} \bar{C}_{m} \int_{0}^{\infty} \frac{\bar{r}_{1}^{\prime} \bar{y}_{2}^{\prime} n^{\prime} \mathrm{d} n^{\prime}}{1+\bar{C}_{m}\left(\frac{\nu_{\infty}}{\nu_{0}}-1\right) E_{1}^{\prime}}=N_{0} \\
& -23-
\end{aligned}
$$


and

$$
2 \pi \rho_{\infty}\left(r_{1 / 2}\right)_{u}^{2} \bar{U}_{m} \int_{0}^{\infty} \frac{f_{2}^{\prime} n^{\prime} d n^{\prime}}{1+\bar{C}_{m}\left(\frac{\rho_{\infty}}{\rho_{0}}-1\right) f_{1}^{\prime}}=K_{e} z M_{0}^{1 / 2} \rho_{\infty}^{1 / 2}
$$

Denoting the integrals in eqs. $(42-44)$ as $I_{1}, I_{2}$, and $I_{3}$, respectively, and assuming uniform profiles at the jet exit, these three equations become

$$
\begin{aligned}
& \frac{U_{0}^{2}}{\bar{U}_{m}^{2}}=2 \frac{\left(r_{1 / 2}\right)_{u}^{2}}{r_{\varepsilon}^{2}} I_{1} \\
& \frac{U_{o}}{\bar{U}_{m} \bar{C}_{m}}=2 \frac{\left(r_{1 / 2}\right)_{u}^{2}}{r_{\varepsilon}^{2}} I_{2} \\
& \frac{U_{0}}{\bar{U}_{m}}=\frac{2 \pi}{K_{e} e^{2}} \frac{\left(r_{1 / 2}\right)_{u}^{2}}{r_{\varepsilon}} I_{3}
\end{aligned}
$$

These three equations are easily solved simultaneously to give

$$
\begin{aligned}
& \frac{1}{\overline{\mathrm{C}}_{\mathrm{m}}}=\frac{\mathrm{K}_{e^{z}}}{\pi^{1 / 2} \mathrm{r}_{\varepsilon}} \frac{\mathrm{I}_{2}}{\mathrm{I}_{3}} \\
& \frac{\mathrm{U}_{\mathrm{O}}}{\overrightarrow{\mathrm{U}}_{\mathrm{m}}}=\frac{\mathrm{K}_{\mathrm{e}}{ }^{z}}{\pi^{1 / 2} \mathrm{r}_{\varepsilon}} \frac{\mathrm{I}_{1}}{\mathrm{I}_{3}}
\end{aligned}
$$

and

$$
\left(r_{1 / 2}\right)_{u}=\frac{K_{e} z}{(2 \pi)^{1 / 2}} \frac{I_{l}^{1 / 2}}{I^{3}}
$$


The behaviors of $1 / \bar{C}_{m}, U_{o} / \bar{U}_{m}$, and $\left(r_{1 / 2}\right)_{u}$ assuming a value of $R=1.17$ are shown in fig. 7 of Chen and Rodi's monograph [5] for values of $\rho_{0} / \rho_{\infty}$ ranging from 3.3 to 0.07 . Our primary interest here is the behavior of $\bar{C}_{m}$ as a function of $z / r_{0}$ and $\rho_{0} / \rho_{\infty}$. In order to make this calculation, it is necessary to solve for values of $I_{2} / I_{3}$ as a function of $\bar{C}_{m}\left[\left(\rho_{\infty} / \rho_{0}\right)-1\right]$. Solutions have been obtained by numerical integration on a minicomputer. Figure 2 shows a plot of the results. Using this curve, the desired results for $\bar{C}_{m}$ as functions of $z / r_{0}$ and $R_{\rho}$ can be calculated using eq. (48). The results of calculations for the flows studied here are described in section 5 .

2.3 Effects of Buoyancy and Coflowing Surrounding Gases on Mixing in Axisymmetric Turbulent Jets

For the experiments to be reported here, it has been necessary to utilize a coflow instead of quiet ambient surroundings in order to insure that no dust particles enter the measurement volume. Rayleigh light scattering intensity measurements, which are used as the concentration diagnostic, are extremely sensitive to the strong scattering which occurs from small particles (Mie scattering). In order to limit gas volume flow rates, a relatively small flow system is utilized. Unfortunately, this places a relatively low limit on the volume flow rate of coflow gas which can be used; and, as will be shown, this, in turn, limits the Re which can be utilized for the jets to relatively low values. Since the inftial flow velocities are limited, it is possible that buoyancy effects can start to dominate these originally momentum-driven flows as the jets move further downstream. For these reasons, it is necessary to consider the effects of buoyancy and the presence of a surrounding coflow on the jet measurements. 
A crude estimate of buoyancy effects can be obtalned using an integral analysis approach. The integral momentum equation (eq. (16)) can be modifled to Include buoyancy effects [12] by writing the jet momentum at $z$ as

$$
2 \pi \int_{0}^{\infty} \overline{p U}^{2} r d r=M_{0}+2 \pi g \int_{0}^{z} \int_{0}^{\infty}\left(p_{\infty}-\bar{\rho}\right) r d r d z^{\prime}=M_{0}+B(z),
$$

where the flow momentum will increase for a positively buoyant jet ( $R_{\rho}<1$ ) and decrease for a negatively buoyant jet $\left(R_{\rho}>1\right)$. The several different types of flow which can result are described by Chen and Rod1 [5].

Equation (51) can be solved approximately in order to obtain an expression for the ratio of momentum imparted to the flow at the nozzle to that for a given $z$ location due to buoyancy $\left(R_{m}=M_{0} / B(z)\right)$. In order to generate this solution, it is assumed that there is no coflow, self-similarity holds throughout the flow fleld, and that the centerline concentration is described by eq. (24) with $\left(z_{0}\right)_{c}=0$. The last term of eq. (51) can be rewritten as

$$
B(z)=2 \pi g \int_{0}^{z} \int_{0}^{\infty}\left[\rho_{\infty}-\frac{\rho_{\infty}}{1+\bar{C}_{m}\left(\frac{\rho_{\infty}}{\rho_{0}}-1\right) f_{1}}\right] z^{\prime 2} n d n d z^{\prime}
$$

Expanding in $\bar{C}_{m}$, Ignorling higher order terms, and substituting eq. (24) gives

$$
B(z)=\pi g \rho_{\infty} \frac{r_{\varepsilon}}{K_{c}}\left(\frac{\rho_{\infty}}{\rho_{0}}-1\right) z^{2} \int_{0}^{\infty} f_{1} n d n
$$

The half angle $(\theta)$ of varlable density jets which obey eq. (24) has been found experimentally $[5,19]$ to be Independent of $R_{\rho}$. Using the experimentally determined value and assuming Gaussian radial profiles, the value of the remaining integral in eq. (53) can be expressed as 


$$
\int_{0}^{\infty} e^{-0.693 \frac{n^{2}}{\tan ^{2} \theta}} n d n=\frac{\tan ^{2} \theta}{2 \times 0.693}
$$

The final form of $B(z)$ then becomes

$$
B(z)=2.27 g \frac{\Sigma_{\varepsilon}}{K_{c}} \rho_{\infty}\left(\frac{\rho_{\infty}}{\rho_{0}}-1\right) z^{2} \tan ^{2} \theta
$$

Assuming uniform profiles at the jet exit, $M_{0} 1$ is given by $\pi r_{0}^{2} \rho_{0} U_{0}^{2}$ and $R_{m}(z)$ becomes

$$
R_{m}(z)=\frac{1.39 I_{\varepsilon} U_{0}^{2} K c}{g z^{2} \tan ^{2} \theta}\left(\frac{\rho_{0}}{\rho_{\infty}-\rho_{0}}\right)
$$

The form of $R_{m}(z)$ is reminiscent of that for the jet Froude number as defined by Chen and Rod1 [5],

$$
F=\frac{U_{0}^{2}}{g D_{0}} \frac{\rho_{0}}{\rho_{\infty}-\rho_{0}}
$$

The $81 g n$ and magnitude of $R_{m}(z)$ are indicators of the type of flow behavior expected at varlous downstream positions for variable density jets. If the absolute magnitude of $R_{m}(z) \gg 1$ the flow is dominated by the initial momentum at the nozzle and the behavior is that of a forced jet. As the absolute magnitude of $R_{\mathrm{m}}(2)$ approaches 1 this is no longer true. For positive values of $R_{m}(z)$ (positively buoyant jets), the momentum of the flow increases as $R_{m}(z)$ decreases and at downstream distances where $R_{m}(z)=1$ a mfxed flow behavior between that for purely forced jets and buoyant plumes occurs. As $R_{\mathrm{m}}(z)$ becomes still smaller the flow behavior becomes that for a buoyant plume. On the other hand, for $R_{m}(z)<-1$ (negatively buoyant $j e t$ ), the total flow momentum is decreasing as the downstream distance increases and the 
absolute magnitude of $R_{m}(z)$ decreases. For $R_{m}(z)=-1$, the downward momentum due to buoyancy is exactly balanced by the upward momentum Imparted to the fluid at the nozzle. As this point $\left(z_{\max }\right)$ the jet will stop rising and jet fluid will begin to fall. This type of flow behaves as a fountain.

Equation (56) can be used to generate an estimate for this helght as

$$
z_{\max }=\left[\frac{1.39 \mathrm{r}_{\varepsilon} U_{0}^{2} \mathrm{~K} c}{\operatorname{gtan}^{2} \theta}\left(\frac{\rho_{0}}{\rho_{0}-\rho_{\infty}}\right)\right]^{1 / 2}
$$

The problem of jets having negative buoyancy has been investigated by Turner [24]. Dimensional arguments were used to show that $z_{\text {max }}$ can be written as

$$
z_{\max }=\frac{\mathrm{K}_{\mathrm{m}} \mathrm{r}^{1 / 2} U_{0}}{g^{1 / 2}}\left(\frac{\rho_{0}}{\rho_{0}-\rho_{\infty}}\right)^{1 / 2}
$$

where $K_{m}$ is a constant to be experimentally determined. Equations (58) and (59) are very similar except for a slightly different dependence on density and the values of constants. From studies on mixing of salt water fets into pure water, Turner measured a value of $\mathrm{K}_{\mathrm{m}}=2.46$.

In section 5.5, eq. (56) is used to estimate the effects of buoyancy on the variable density jets investigated in this study. The fountain-like behavior predicted by eqs. (58) and (59) has been observed and is discussed in section 5.2 .

It should be cautioned that eqs. (56) and (58) are based on assumptions which cannot be correct throughout the flow fleld. For this reason, they should be considered only as estimates for $R_{m}(z)$ and $z_{\max }$. These equations 
only apply to the behavior of the average concentration. The effects of buoyancy on turbulent concentration fluctuations are not addressed and must be considered as unknown.

The use of a secondary flow within an enclosure as the surrounding gas for the primary turbulent jet can strongly perturb the mixing behavior from that characteristic of free jets. Such modifications can be conveniently categorized as those which arise only from the presence of an infinite surrounding flow and those which occur due to the presence of a wall which serves to contain the secondary flow. The latter modifications can be due to several different effects including lack of enough mass volume flow in the secondary stream to meet the entrainment capabilities of the jet [18], expansion of the primary jet until it strikes the enclosing walls [18], and the development of pressure gradients within the enclosure [12].

The behavior of turbulent jets flowing collaterally into a secondary air flow of infinite extent has been discussed extensively in the monographs of Harsha [12] and Abramovich [25]. The nondimensionalized parameter which is utilized to characterize this type of flow is $m=U_{\infty} / U_{0}$. Our primary interest here are flows for which $0<\mathrm{m} \ll<1$. Experiments summarized in the two monographs lead to the following conclusions concerning the mixing behavior of this type of flow:

1. Self-similarity is not possible, but is approached for constant density flows when $\mathrm{m} \ll 1$; 
2. Radial profiles of concentration and velocity at moderate downstream distances are locally simflar even when the flow is not self-simflar;

3. As the value of $m$ is 1ncreased, the spreading rate of the jet decreases and has a nonlinear dependence on downstream distance;

4. The potential core lengths of the turbulent jets increase as $m$ is Increased; and

5. The potential core length increases with Re (as is the case for free jets) for constant values of $\mathrm{m}$.

Abramovich [25] has given approximate formulas for the variation of these various properties of the flow with $\mathrm{m}$.

The effects of limiting the mass flow rate of the secondary gas have been considered by several workers. The qualitative discussion given by Thring and Newby [18] 1s very 1nstructive. For cases where the mass flow rate of the secondary gas is large enough to meet the entrainment requirements of the jet as far downstream as the point where the outer boundary of the jet strikes the walls of the enclosure, it is expected that the jet w1ll behave much like a jet into a coflow until this point is reached. However, if the secondary mass flow rate is not sufficient to meet the entrainment capabilicy of the jet, fluid will be drawn from downstream regions and recirculation eddles w1ll develop in the secondary flow. An estimate of the downstream location $\left({ }^{d}\right)$ where recirculation will develop can be obtained using R1cou and Spaldings' entra1nment law [21] (eq. (37)). After mak1ng the appropriate subst1tutions, eq. (37) becomes 


$$
\mathrm{z}_{\mathrm{d}}=6.25\left(\frac{\dot{\mathrm{m}}_{\infty}+\dot{\mathrm{m}}_{0}}{\mathrm{~m}_{0}}\right) \mathrm{r}_{\varepsilon}
$$

where $\dot{\mathrm{m}}_{\infty}$ is the mass flow rate for the secondary gas. Th1s relation is similar to one given by Thring and Newby [18] using an earlier experimentally determined expression for entrainment.

Later work by Becker, Hottel, and Willlams [26] has provided a quantitative means for dealing with enclosed primary jets entering secondary flows of the same density. These workers utilized a simflarity parameter which they named the Craya-Curtet number $(C t)$ in recognition of Craya and Curtet $[27,28]$ who were the first researchers to ut1lize a rigorous similarity analysis to treat this problem. Becker et al [26] 1ntroduced two velocities known as the kinematic velocity, $U_{k}$, and the dynamic mean velocity, $U_{d}$, which for uniform primary and secondary flows are defined as

$$
U_{k}=\frac{U_{0} r_{0}^{2}+U_{\infty}\left(r_{e}^{2}-r_{0}^{2}\right)}{r_{e}^{2}}
$$

and

$$
u_{d}=\left[\frac{U_{0}^{2} r_{0}^{2}+U_{\infty}^{2}\left(r_{e}^{2}-r_{0}^{2}\right)}{r_{e}^{2}}-\frac{U_{\infty}^{2}}{2}\right]^{1 / 2}
$$

where $r_{e}$ is the radius of the cylindrical enclosure. $U_{k}$ is the average velocity for the total flow within the enclosure and the square of $U_{d}$ is a measure of nonuniformity in the momentum flux distribution at $z=0$ across both the primary and secondary flows. In terms of $U_{k}$ and $U_{d}$,

$$
C t=\frac{u_{k}}{\left(u_{d}^{2}-\frac{u_{k}^{2}}{2}\right)^{1 / 2}}
$$


Experimentally, it has been found that recirculation only occurs for $C t<0.67$ At higher values of $C t$ the jet strikes the walls of the enclosure before recirculation eddies begin to develop. Becker et al [26] discovered that measurements of $\left(r_{1 / 2}\right)_{u},\left(r_{1 / 2}\right)_{c}, 1 / \bar{U}_{m}$, and $1 / \bar{C}_{m}$ as functions of $z / r_{0}$ were indistinguishable from those for a free jet when $C t$ was equal to 0.67 . These workers reported extensive measurements of the flow fields for velocity and jet fluid in an axisymetric turbulent jet flowing into a secondary flow enclosed within a cylindrical enclosure. Their findings for downstream flow regions can be summarized as follows:

1. Radial profiles of velocity for $C t<0.67$ are not locally similar. Interestingly, for values of $\bar{U} /\left(\bar{U}_{1 / 2}\right)_{u}$ there is a universal curve for measurements made in the flow region extending from the jet axis to $r \approx\left(r_{1 / 2}\right)_{u}$. Local similarity only fails in the outer radial regions of the jet;

2. The values of $\left(\bar{U}_{1 / 2}\right)_{u}$ for a given value of $z / r_{0}$ increase with decreasing $C t$;

3. Centerline velocity fallof $f$ as a function of $z / r_{0}$ is faster for smaller values of $C t$;

4. As expected, the location of recirculation eddies in the secondary stream move upstream as $C t$ is decreased; and

5. For values of $C t>0.67$ the jet fluid concentration is found to display local simflarity in the radial direction. 
These conclusions are consistent with a picture in which mixing behavior Is dominated by recirculation effects for $C t<0.67$, behaves much like that for a free jet for $C t=0.67$, and is dominated by the value of $U_{\infty} / U_{0}$ for $C t>0.67$. With regard to flows where $\mathrm{Ct}>0.67$, the behavior of the experimental data obtained by Becker et al [26] is in qualitative agreement with the conclusions given above for the dependence of flow behavior on $\mathrm{U}_{\infty} / \mathrm{U}_{0}$.

Steward and Guruz [29] have extended the work of Becker et al [26] to variable density flows. Their analysis indicates that the similarity parameter Ct defined by eq. (63) is valid for cases where initially uniform flows of primary and secondary gases have different densities if the parameters $U_{k}$ and $U_{d}$ defined by eqs. (61) and (62) are replaced by the density weighted variables

$$
U_{k}=\frac{r_{0}^{2} U_{0} \rho_{0}+\left(r_{e}^{2}-r_{o}^{2}\right) U_{\infty} \rho_{\infty}}{r_{e}^{2} \rho^{\prime}}
$$

and

$$
U_{d}=\left(\frac{r_{0}^{2} U_{0}^{2} \rho_{0}+\left(r_{e}^{2}-r_{0}^{2}\right) U_{\infty}^{2} \rho_{\infty}}{r_{e}^{2} \rho^{\prime}}-\frac{U_{\infty}^{2}}{2} \frac{\rho_{\infty}}{\rho^{\top}}\right)^{1 / 2}
$$

where

$$
\rho^{\prime}=\frac{r_{0}^{2} U_{0} \rho_{0}+\left(r_{e}^{2}-r_{0}^{2}\right) U_{\infty} \rho_{\infty}}{r_{0}^{2} U_{0}+\left(r_{\varepsilon}^{2}-r_{0}^{2}\right) U_{\infty}}
$$

These authors reported experimental measurements for heated and particlemarked air jets flowing into a secondary flow of air which confirmed that treating their data in terms of the variable density $C t$ gave results which were in good quantitative agreement with those reported for constant density flows by Becker et al [26]. 


\section{EXPERIMENTAL SYSTEMS AND DATA TREATMENT}

Two different optical experiments have been performed during the course of this study. Shadowgraphy was used to visualize the globrl flow fleld. Quant1tat1ve measurements of the real-t1me concentration behavior for the fet fluld were made along the jet centerline using the recently developed technique of laser-1nduced Raylelgh light scattering [1,2]. For both experiments, the same flow system and conditions were ut1lized. The following sections describe the flow system as well as the experimental systems utilized for shadowgraph flow v1sualization and for laser-1nduced Raylelgh light scattering measurements of concentration.

\subsection{Flow System}

The flow system employed for this study is the same as used in our earlier study on the feasiblitty of Raylelgh light scattering as a concentration probe in variable density flows $[1,2]$. An axisymmetric turbulent jet was formed in the center of a square cylinder through which a slow coflow of a second gas was passed. The enclosure served to prevent dust particles in the laboratory from reaching the observation volume for the Rayleigh light scattering measurement.

A $61 \mathrm{~cm}$ length of $6.35 \mathrm{~mm}$ Inside diameter brass tubing formed the jet flow. The tubing is tapered to a fine edge at the jet exit. A ballast chamber was attached to the upstream end of the pipe in order to dampen pressure fluctuations. The plpe was long enough to ensure that fullydeveloped turbulent pipe flow occurs before the gas exits the nozzle. The 
axis of the jet was aligned vertically and the flow direction was upward so that fluid flow was against the force of gravity.

A $10.4 \times 10.4 \times 61 \mathrm{~cm}^{3}$ square enclosure formed from $5 \mathrm{~mm}$ thick optical crown glass served to contain the secondary flow. The coflow gas passed through a bed of polystyrene balls and a wire gauze in order to create a homogeneous flow before entering the enclosure well upstream of the jet nozzle exit.

The primary and secondary gas flows were prefiltered using 0.3 micron filters. Alr was supplied by a regulated house airline while the other gases utilized were obtained from cylinders. Table 1 lists the suppliers and stated purities for the gases utilized in this study.* Gas volume flow rates were controlled and measured by Fischer and Porter Flowrator meters. Pressure gauges monitored the pressure drop through the flow system and, where necessary, the volume flow rates were corrected to one atmosphere. Pressure drops within the enclosure were assumed to be negligible.

Values of $\mathrm{Re}$ for the jet flows were calculated using the expression

$$
\operatorname{Re}=\frac{\rho_{0} U_{0} D_{0}}{\mu_{0}}
$$

$U_{0}$ is the velocity of the primary gas at the nozzle assuming a uniform flow profile and $D_{0}$ is the diameter of the nozzle. Values of $\rho_{0}$ and $\mu_{0}$ wers taken from standard references as described in [30]

\footnotetext{
"Certain commercial equipment, instruments, or materials are identified in this paper in order to adequately specify the experimental procedure. Such identification does not imply recommendation or endorsement by the National Bureau of Standards nor does it imply the instruments or equipment are necessarily the best available for the purpose.
} 
Since only the center region of the expanding Gaussian beam was collimated, the $76 \mathrm{~mm}$ beam which resulted had a relatively uniform light intensity distribution.

The collimated laser light pulse next passed through the glass enclosure where it interacted with the flow field formed by the turbulent jet and the slowly flowing secondary gas. After passing through the far wall of the glass enclosure, the beam traveled $\approx 2.2 \mathrm{~m}$ before striking a thin diffuser which was placed within the focal plane of a HP 197A oscilloscope camera. Images of the shadowgraph were recorded using Type 107 Polaroid film. Camera shutter times of $0.033 \mathrm{~s}$ were utilized to insure that only one image of the flow field was recorded on each photograph. F-stop values were chosen to produce adequate exposure of the film and generally fell in the 8-16 range.

\subsection{System for Rayleigh Light Scattering Measurements of Concentration}

The use of Rayleigh light scattering for concentration measurements in binary gas mixtures has been discussed in detail in our earlier works $[1,2]$. The basis of the technique is the dependence of the intensity of Rayle1gh light scattering on the index of refraction of a gas. In general, the Indices of refraction for different gases vary. For mixtures of two gases, the scattering intensity is found to be linearly proportional to the mole fractions of the gases which form the mixture. This observation along with the relation

$$
x_{1}+x_{2}=1
$$


where $x_{1}$ and $x_{2}$ are the mole fractions for the two gases, allows the mole fraction of a gas to be determined following calibration of the scattering intensity by making measurements in pure gases 1 and 2 .

A full description of the experimental system employed for laser-induced Rayleigh light scattering measurements of real-time, spatially-resolved concentration fluctuations in turbulent flows of binary gas mixtures is available [1,2]. Only a brief description of the experiment is repeated here. Figure 4 shows a schematic for the experimental system. Rayleigh light scattering was induced by the $8 \mathrm{~W}, 488 \mathrm{~nm}$ output of the Ar ion laser. The laser beam was focused by a lens to a narrow waist $(\approx 0.05 \mathrm{~mm})$ at the desired observation location within the flow enclosure. Scattered light was collected at $90^{\circ}$ to the laser beam by a f/2 optical system which imaged the scattered light onto a $0.4 \mathrm{~mm}$ pinhole (the pinhole replaced the slits which were used in the earlier work $[1,2])$. Since the laser beam was focused onto the pinhole as a 1:1 image, the observation volume was a cylinder having an approximate diameter of $0.05 \mathrm{~mm}$ and length of $0.4 \mathrm{~mm}$. The resulting observation volume is $0.0008 \mathrm{~mm}^{3}$.

After passing through the pinhole, the light was once again collimated and then focused onto the photocathode of an EMI $9781 \mathrm{~B}$ photomultiplier tube (PMT). A narrow bandpass optical filter centered at $488 \mathrm{~nm}$ which was used in the original experiments $[1,2]$ was not employed in this study. The current output of the PMT was converted to a voltage by shunting it to ground across a load resistor. The voltage output of the amplifier was then digitized and stored in the memory of a minicomputer. In general, the data collection rate was $10 \mathrm{kHz}$ and 32,768 individual measurements were taken. After collection, the data was stored on a hard disk for later analysis. 
Recorded time series of concentration behavior were used to calculate time-averaged values of average, rms, skewness, and kurtosis (flatness) at various downstream positions along the jet centerline. Note that for a Gaussian distribution the skewness is zero and the kurtosis is 3 . Results are reported in terms of both mole (X) and mass fraction (Y) of jet gas. The average and rms mole fractions were converted to mass fraction results using the procedure described by Birch et al [10] in exactly the same way as previously described [1,2]. Mathematical techniques exist for correcting the raw data for the effects of electronic shot noise when calculating the moments of the concentration distribution $[1,2,10,33]$. These procedures were employed in this work even though such corrections were generally small.

One set of centerline measurements were recorded for a He/air free jet. Light scattering measurements for this flow. were heavily contaminated by the presence of particles in the laboratory air which were entrained into the jet flow. The resulting time-resolved light intensity measurements showed the jet fluid concentration fluctuation behavior from the Rayleigh light scatterfing along with numerous superimposed sharp spikes due to Mie scattering.

Some methods have been developed using computer algorithms to treat Rayleigh light scattering data records which are contaminated by :Le scattering [34]. However, for the limited free jet data recorded during this study a brute force approach was chosen. A stored data record of 32,768 points was visually examined and one hundred random concentration measurements were chosen for which no particle interferences were present. The measurements were then averaged and the rms calculated to give $\bar{X}_{m}$ and $x_{m}^{\prime}$. Values of $\bar{Y}_{m}$ and $Y_{m}^{\prime}$ were then calculated as described above. 


\section{EXPERIMENTAL FINDINGS}

\subsection{Shadowgraphy}

Shadowgraphy has been used to visualize all of the flows listed in table 2 except those for the $\mathrm{CF}_{4}$ jets. Figures 5-19 show examples of the photographs which have been recorded. Shadowgraphs for each jet/coflow gas pair combination are discussed below. The discussion is ordered from low to high values of $R_{\rho}$.

$$
\text { 4.1.1 Helium Turbulent Jet into Air }\left(R_{\rho}=0.14\right)
$$

Figure 5 shows a composite of three shadowgraphs recorded at different times and downstream distances for the helium turbulent jet at $\operatorname{Re}=3950$ flowing into a secondary flow of air. An axis system has been included which indicates the radial distance from the centerline in terms of $r / r_{0}$ and the nondimensionalized downstream distance, $z / r_{0}$. The diffuse lines which are evident in regions outside of the turbulent jet are due to index of refraction variations within the glass enclosure which surrounded the entire flow field. Since the light source was a laser, coherent effects are possible. Close inspection of fig. 5 reveals that interference effects are present in the photograph. Fortunately, these effects are not large enough to negate conclusions which are drawn concerning the flow behavior.

Several observations can be made concerning the flow behavior visualized in fig. 5. Even though axisymmetric turbulent jets are known to have large scale structure and to display a great deal of intermittency in the outer 
regions of the jet, the outline of the jet observed in this integrated view of the flow field is relatively smooth. This is especially evident from the fact that three independent shadowgraphs can be superimposed to give an apparently continuous view of the flow. This observation is somewhat surprising since the flow field has been "frozen" by the short light pulse and large scale structures should be easily observed.

The spreading behavior of the outer boundary of the helium jet is evident from fig. 5. As the flow moves downstream from the nozzle there is a continuous increase in the diameter (D) of the jet. After a short distance downstream from the nozzle, the spreading rate appears to be linearly dependent on downstream distance. At a downstream distance of $z / r_{0}=40$ the apparent value of $\mathrm{D}$ is roughly $20 \mathrm{r}_{0} \cdot$

Turbulent fluctuations of density in the jet are evident from the cellular appearance of the shadowgraphs throughout the jet region. At certain locations on the outer boundary of the jet, what appear to be localized vortices can be seen. The fluctuations of density in this Elow fleld extend upstream to a point just above the nozzle exit and the "roll up" of the jet fluid into vortices is evident within one jet radius of the nozzle exit.

As will be discussed later, there were experimental indications that the enclosed helium jet interacted with the surrounding glass walls. For this reason, shadowgraphs were recorded for the helium jet Elowing freely into the laboratory. Figure 6 shows the results. Once again, three independent photographs have been superimposed. The image appears clearer than that in E1g. 5 since the variations in the index of refraction due to the glass walls are 
absent. Comparison of figs. 5 and 6 reveals no obvious differences between the shadowgraphs for the free and enclosed helium jets.

\subsubsection{Methane Turbulent Jet 1nto Air $\left(R_{\rho}=0.55\right)$}

Figure 7 shows four independent shadowgraph visualizations of the $\mathrm{CH}_{4}$ turbulent jet flowing into a secondary flow of air. There are obvious differences in the appearance of this flow field as compared to those for the He/air flows (flgs. 5 and 6 ). The $\mathrm{CH}_{4}$ jet seems to spread radially much more slowly than the helium jet. This is reflected in the observation that this jet has not grown to a width of $7.5 \mathrm{~cm}$ (the observation diameter) by $55 \mathrm{r}_{0}$ downstream as compared to the He/air flow which reaches this width at $\approx 45 \mathrm{I}_{0^{\circ}}$ At $z / r_{0}=40$, the visualized diameter of the turbulent jet is roughly $16 r_{0}$.

The turbulent structure of the $\mathrm{CH}_{4}$ air shadowgraph also has a different appearance than those seen in the He/air flows. Contrast the "cellular" structure for the lighter jet with the "stringy" appearance of the turbulent structure in fig. 7. Large scale structures in the outer radial regions of the $\mathrm{CH}_{4}$ /air turbulent jet also are more pronounced. A potential core region is clearly evident near the nozzle. Indications of vortex roll up on the outer edge of the flow field are not seen until a downstream distance of $\approx 1.6 \mathrm{r}_{0}$

$$
\text { 4.1.3 Propane Turbulent Jet into Carbon Dioxide }\left(\mathrm{R}_{\rho}=1.02\right)
$$

The primary and secondary flow gases for this combination have nearly the same densities and the jet is non-buoyant. In this regard, it should be 
identical to jets of air which have been widely used in earlier turbulent flow studies.

Many of the trends already noted on going from the small value of $R_{p}$ of the He/air flow to the higher value of $\mathrm{R}_{\rho}$ for the $\mathrm{CH}_{4}$ /air flow continue to develop as $R_{\rho}$ is increased to that for this gas pair. Figure 8 shows the superposition of four independent shadowgraph visualizations of the flow fleld. The observed diameter for a given downstream distance is reduced from that found for the jets having smaller values of $R_{\rho}$. The value of $D$ at $z / r_{0}=40$ is $14 r_{0}$. The turbulent structure is more pronounced and appears to be very "stringy". Large scale structures are clearly seen near the edge of the jet. A long potential core can be discerned and the first indication of vortex roll up on the outer edge of the jet occurs at roughly $1.9 r_{0}$.

\subsubsection{Propane Turbulent Jet into Air $\left(R_{p}=1.55\right)$}

Figure 9 shows four superimposed shadowgraph views of the $\mathrm{C}_{3} \mathrm{H}_{8} /$ air jet/coflow combination for nearly the same $\operatorname{Re}(\operatorname{Re}=3960)$ as used for the He/air, $\mathrm{CH}_{4}$ /air, and $\mathrm{C}_{3} \mathrm{H}_{8} / \mathrm{CO}_{2}$ flows. This jet has an appearance which is very similar to that shown in fig. 8 for the $\mathrm{C}_{3} \mathrm{H}_{8} / \mathrm{CO}_{2}$ case. However, the turbulent structure seems to be less developed and to not have the same degree of small scale structure as the combination with the smaller value of $R_{\rho}$. For $z / r_{0}=40$, the value of $D$ is estimated to be $14 r_{0}$, the same value as found for the $\mathrm{C}_{3} \mathrm{H}_{8} / \mathrm{CO}_{2}$ flow. The distance from the nozzle to the first vortex roll up is $\approx 2.5 \mathrm{r}$. 
As indicated in table 2, higher velocity propane flows have also been studied. Figures 10 and 11 show superimposed shadowgraphs for $\operatorname{Re}=7930$ and 11880, respectively. The downstream spreading behavior for the different Re flows (figs. 9-11) appear to be nearly the same (e.g., a value of $D \approx 14 r_{0}$ is estimated for the three jets at $\left.z / r_{0}=40\right)$. However, the turbulent structure of these jets in the higher Re flows appears more developed and to extend to smaller spatial scales.

The development of the $\mathrm{C}_{3} \mathrm{H}_{8} /$ air jets in the potential core regions has a strong dependence on $\operatorname{Re}$. As the Re is increased, initial vortex roll up of the outer portion of the jet fluid moves closer to the nozzle (values of $1.3 r_{0}$ and $1.1 r_{0}$ are estimated for $\operatorname{Re}=7930$ and $\operatorname{Re}=11880$, respectively). A well-developed potential core, which is very evident as a bright region near the nozzle in fig. 9, is found for the lowest Re jet. The length of the potential core decreases with increasing $\operatorname{Re}$ and has nearly disappeared in the $\operatorname{Re}=11880$ flow (fig. 11$)$.

\subsubsection{Sulfur Hexafluoride Turbulent Jet into Air $\left(R_{\rho}=5.11\right)$}

The $\mathrm{SF}_{6} /$ air combination has a jet/coflow density ratio of $\mathrm{R}_{\rho}=5.11$. For $\operatorname{Re}=3950$, table 2 indicates that this jet should form a fountain. Figures 12-14 are individual shadowgraphs of this flow taken at progressively higher values of $z / r_{0}$. It was impossible to superimpose shadowgraphs since there was a great variability in the appearance of the flow field for photographs taken at the same downstream position. Figure 12 shows that this flow has an extremely long potential core region and distance between the nozzle and the onset of vortex roll up $\left(z / r_{0} \approx 3.4\right)$. Once turbulence is initlated, it has a very similar appearance to that observed in the $\mathrm{C}_{3} \mathrm{H}_{8}$ turbulent jets. 
Jet flufd ( $\mathrm{SF}_{6}$ and air mixture) can be seen in regions well outside of the jet in fig. 12. Indications for the presence of $\mathrm{SF}_{6}$ can even be seen in the secondary flow at locations well below the nozzle. The presence of $\mathrm{SF}_{6}$ in the coflow is even more evident in fig. 13 which is taken further downstream. The turbulent jet is still visible in the center, but the concentration variations in the secondary flow region are now quite intense. The source of jet fluid in the secondary flow is shown to be due to a fountain effect in fig. 14. This shadowgraph clearly shows the jet fluid reaching a maximum height and then falling back down through the secondary gas. The maximum downstream distance attained by the turbulent jet is estimated to be $z_{\max } / r_{0}=38$.

Measurements have also been made for $\mathrm{SF}_{6} /$ air flows having higher $\mathrm{Re}$. Figures 15 and 16 display shadowgraphs for $\operatorname{Re}=7890$ and 11860 . For these two flows, it has been possible to once again superimpose independent shadowgraphs in order to get an overall view of the flow field. In contrast to the fet with $\operatorname{Re}=3950$, no jet fluid is observed in the secondary flow and the turbulence structure has an appearance similar to that already described for other jet/coflow gas pair combinations.

Modifications in the appearance of the shadowgraphs for the two higher Re flows are similar to those already discussed in section 4.1 .4 for the $\mathrm{C}_{3} \mathrm{H}_{8} / \mathrm{alr}$ flows. Downstream turbulent mixing apparently becomes more intense with smaller scales, but the radial growth behavior of the jet is Independent of $\operatorname{Re}\left(D \approx 10 \mathrm{r}_{0}\right.$ for $\left.z / r_{0}=40\right)$. The visible lengths of the potential cores for the variable Re jets, which can be seen in figures 15 and 16 , decrease on going from $\operatorname{Re}=7890$ to $\mathrm{Re}=11860$. The distance from the nozzle to the f1rst appearance of vortex roll up is estimated to be $2.8 \mathrm{r}_{0}$ for $\operatorname{Re}=7860$ and 2.2 $r_{0}$ for $\operatorname{Re}=11860$ 
4.1.6 Sulfur Hexafluoride Turbulent Jet into Helium $\left(R_{\rho}=37.0\right)$

This flow has only been investigated for $\operatorname{Re}=3950$. As might be expected, this jet behaves much like the low $\operatorname{Re} \mathrm{SF}_{6} /$ air flow in that it has a fountain behavior in which the dense $\mathrm{SF}_{6}$ gas falls through the very low density secondary He flow. Figures $17-19$ show shadowgraphs for the $\mathrm{SF}_{6} / \mathrm{He}$ flow field taken at three different downstream positions. The top of the fountain at $z / r_{0} \approx 44$ is obvious in $\mathrm{fig} .19$ and the presence of $\mathrm{SF}_{6}$ in the He coflow can be seen in figs. 17 and 18 .

This jet spreads very slowly in comparison with the other jet/coflow gas pairs already discussed. The shadowgraphs indicate that while concentration fluctuations are present in the flow field, the flow does not have the fine structure characteristic of turbulent flow.

Experimentally, it has been found that the appearance of shadowgraphs taken near the nozzle for this flow vary from photograph to photograph. For this reason, only an estimate for the distance from the nozzle to the first vortex roll up is possible. It is clear, however, that this distance is considerably further downstream than those found for the other gas pairs investigated.

4.1.7 Summary of Trends in Mixing Behavior with Changes in $R_{\rho}$ and $\operatorname{Re}$ Derived from Shadowgraphs

In drawing conclusions concerning mixing behavior from the shadowgraphs shown in figs. 5-19, it must be kept in mind that the technique inherently gives an integrated view of the flow field. The observed shadowgraph is not 
necessarily correlated with the local structure of the jet flow field. Varlations in indices of refraction among different gases can also lead to misinterpretation when comparing shadowgraphs for flows of different gas palrs.

Estimation of jet spreading rates from visualization studies should be especially susceptible to such effects since it has not yet been shown that the apparent diameter of the flow field is correlated with the true diameter of the jet. Even though full radial concentration profiles have not been made for all of the jet/coflow combinations, such measurements for $z / r_{0}=31.5$ were previously described for the $\mathrm{CH}_{4}$ /alr case $[1,2]$ and were made for the $\mathrm{SF}_{6}$ air flow at $\operatorname{Re}=7930$ during the course of this study. For both of these flows it was found that the apparent widths of the jets from the time-resolved shadowgraphs were less than estimates of the full widths derived from the radial concentration measurements. This is not surprising since the average concentration of jet gas in the outer radial regions of the flow is due to the passage of relatively infrequent large scale structures having much higher average concentrations [1,2]. An instantaneous photograph is unlikely to capture such structures. Comparison of the photographs and radial concentration measurements do show that the visualized jet widths are roughly constant fractions of the true widths of the jets based on concentration. These results suggest that visualization can be used to make relative comparisons of flows having different values of $R_{\rho}$.

With the above considerations in mind, cautious generallzations based on shadowgraphy may be made concerning the effects of jet/coflow density rat:o and jet $R e$ on the mixing behavior of the axisymetric turbulent jets 1nvesti- 
gated in this study. For a given value of $z / r_{0}$ and Re, the radial extent of the jet is larger for lower values of $R_{\rho}$. As an example of this behavior, table 3 lists values of $D$ obtained visually for $z / r_{0}=40$ which do indeed decrease as $R_{\rho}$ increases. Details of the turbulent structures observed within the different jet flow fields indicate that, for a given Re, turbulent mixing is more intense for jets with smaller values of $R_{\rho}$. The potential core lengths as well as the distance from the nozzle to the first vortex roll up are longer for higher values of $R_{\rho}$. Values of the distances for vortex roll up are included in table 3 for comparison purposes. These conclusions concerning the potential core regions of constant Re jets are consistent with the notion that mixing is more efficient when $R_{\rho}$ is smaller.

The shadowgraphs of the $\mathrm{C}_{3} \mathrm{H}_{8} / \mathrm{air}$ and $\mathrm{SF}_{6}$ /air flows at variable $\mathrm{Re}$ indicate that there is very little dependence on $R e$ for the radial spreading rates of these jets. Values of $D$ at $z / r_{0}=40$ listed in table 3 support this conclusion. On the other hand, the jets are found to have more intense turbulent structures and smaller scales at higher Re. Similarly, the potential core lengths as well as the distance from the nozzle to the point of initial vortex roll up decrease as the Re is increased (table 3).

4.2 Concentration Measurements Along the Centerlines of Variable Density Jets

The laser-induced Rayleigh light scattering technique described in section 3.3 was utilized to record time-resolved jet fluid concentration measurements along the centerlines of the turbulent flows listed in table 2. As part of the calibration procedure used in this technique, it was necessary to record the scattering intensities from the two pure gases under investiga- 
tion. These measurements allow the Rayleigh scattering cross section ratio to be determined for the gas pair. The observed ratios serve as internal checks of the measurement technique. Table 4 lists observed average values of the cross section ratios for the gas pairs investigated along with average deviations based on a large number of independent measurements. Also included are the predicted cross section ratios, which were calculated using known values of gas indices of refraction in the same manner as described in our earlier work $[1,2]$. The variations in the observed value of the scattering intensity ratio for a given gas pair is small and good agreement is found between the experimentally determined values and those predicted.

Figure 20 shows an example of the type of time records which were recorded. These data were recorded for the $\mathrm{CH}_{4} /$ afr flow at a downstream distance of $z / r_{0}=31.5$. The raw data in this figure have been scaled using the scattering intensity calibrations for $\mathrm{CH}_{4}$ and air to give $\mathrm{CH}_{4}$ mole fraction measurements. It is clear from this figure, that the effects of shot noise on the measurement are minimal.

The calculational procedures described in section 3.3 were used to analyze data records such as that shown in fig. 20 in order to calculate values of average and rms concentration in terms of mole and mass fractions as well as the third and fourth moments normalized to give skewness and kurtosis. In the following sections, measurements of the centerline behaviors of $\bar{X}_{m}, \bar{Y}_{m}, X_{m}^{\prime}, Y_{m}^{\prime}, S_{m}$ and $K_{m}$ are discussed. In order to isolate the effects of $\mathrm{R}_{\rho}$ on mixing behavior; wherever possible, comparisons are made for fets having different values of $R_{\rho}$, but for which the Re are the same. The effect of $\operatorname{Re}$ on the centerline mixing behavior is then discussed by comparing the variable $\mathrm{Re}$ jet results for the $\mathrm{C}_{3} \mathrm{H}_{8} / \mathrm{air}, \mathrm{CF}_{4} / \mathrm{a} 1 \mathrm{r}$, and $\mathrm{SF}_{6} /$ air flows. 


\subsection{1 $R_{\rho}$ Dependence of Centerline Average Concentration}

In this section, data are discussed for the He/air, $\mathrm{CH}_{4} / \mathrm{air}, \mathrm{C}_{3} \mathrm{H}_{8} / \mathrm{CO}_{2}$, $\mathrm{C}_{3} \mathrm{H}_{8} / \mathrm{air}, \mathrm{CF}_{4} /$ air, $\mathrm{SF}_{6} /$ air, and $\mathrm{SF}_{6} /$ He jet/coflow combinations at $\operatorname{Re}=3950-3960$. The $\mathrm{SF}_{6} /$ air and $\mathrm{SF}_{6} / \mathrm{He}$ flows at these Re can not be utilized for direct comparisons due to the fountain-like behaviors which can be clearly seen in figs. 14 and 19. Since the $\mathrm{SF}_{6} /$ air jet for $\mathrm{Re}=7890$ has a "normal" turbulent jet appearance (see fig. 15 and the discussion in section 4.1.5), data for this jet/coflow combination are included for comparison purposes with the lower Re data of the other gas pairs.

It is interesting to compare the mixing behaviors of the $\mathrm{SF}_{6} / \mathrm{air}$ and $\mathrm{SF}_{6} /$ He turbulent jets at low Re. Centerline concentration measurements for these two jets are shown in $\mathrm{fig} \cdot 21$ in terms of $Y_{0} / \bar{Y}_{m}$. Concentration data for turbulent jets when plotted in this manner usually have an approximately linear dependence on $z / r_{0}$. These two jets definitely do not have such a dependence. At $z / r_{0} \approx 35$, an abrupt change in the dependence of $Y_{0} / \bar{Y}_{m}$ on $z / r_{0}$ occurs for both. One data point at $z / r_{0}>35$ is included in $\mathrm{fig} \cdot 21$ for the $\mathrm{SF}_{6} /$ air flow. Comparison with figs. 14 and 19 shows that the abrupt changes in centerline concentration behavior for these jets are at roughly the same downstream locations as the tops of the fountains visualized by shadowgraphy.

Experimental data are plotted in fig. 22 as inverse mole fraction versus nondimensionalized downstream distance for the six jet/coflow gas combinations covering a range of $R_{\rho}$ values from 0.14 to 5.1 . The values of $1 / \vec{x}_{m}$ are normalized by multiplying by the mole fraction at $z=0\left(X_{0}\right)$ which is equal to one for all the cases investigated in this study. For values of $z / r_{0}>20$, 
each of the different flows appears to have a linear dependency on downstream distance. The mole fraction data for different gas pairs have been fit to a relation of the form of eq. (12) using a linear least squares fitting procedure. The results of these fits are indicated in fig. 22 by straight Iines and listed in table 5 by reporting the calculated slopes $\left(\mathrm{K}_{\mathrm{x}}\right)$ and virtual origins $\left(z_{0}\right)_{x}$ which result. A clear dependence on $R_{\rho}$ is evident from these results. As $R_{\rho}$ increases, values of $K_{x}$ increase monotonically. Values of $\left(z_{0}\right)_{x}$ also increase with $R_{\rho}$, going from negative values for $R_{\rho}<1$ to positive values for $R_{\rho}>1$.

The same experimental data have also been utilized to calculate the centerline concentration behavior of the jets in mass fraction terms. Figure 23 shows the results for the experimental data taken at $z / r_{0}>20$. All of the data, except that for the He/air case, have an apparent linear falloff with downstream distance. There is a slight curvature in the He/air data which, as will be discussed in section 5.1.1, has been attributed to the jet flow interacting with the walls of the enclosure. In order to test this hypothesis, average concentration measurements along the centerline of a hellum free jet at the same Re have been made in the manner described in section 3.3. Figure 24 compares the results of these measurements with those for the enclosed He/air jet in mass fraction terms. Note that the free jet measurements extend further downstream than those for the enclosed jet. It is clear that the two sets of data agree well in near field regions, but begin to diverge as $z / r_{0}$ 1ncreases. Unlike the enclosed jet, the data for the free jet lie on a straight line. 
Linear least squares fits have been calculated for the different jet/coflow combinations shown in figs. 23 and 24 . Data are reported in the form of eq. (12) by listing values of slopes $\left(K_{y}\right)$ and virtual origins $\left(z_{0}\right)_{y}$ in table 5. The dependencies of $K_{y}$ and $\left(z_{0}\right)_{y}$ on $R_{p}$ are reversed from those found for data plotted in terms of $x_{0} / \bar{x}_{m}$.

The discussion in section 2.2 has indicated that $z / r_{\varepsilon}$, where $r_{\varepsilon}$ is defined by eq. (22) with $\rho_{f}=\rho_{\infty}$, should be a more sultable length parameter for treating variable density flows than $z / r_{0}$. Data for the $s i x$ jets are shown plotted as functions of $z / r_{\varepsilon}$ in $\mathrm{flg} .25$. Data taken from $\mathrm{fIg} \cdot 23$ have been offset by $-\left(z_{0}\right)_{y}$ in order to force each least squares fit to pass through $z / r_{\varepsilon}=0$. This treatment does not give a complete correlation of the experimental results, but it does collapse them into a narrower range of values. A dependence on $R_{\rho}$ still remains. This point is addressed further in the discussion section. Values of $K_{c}$ which result from linear least squares fits of $Y_{0} / \bar{Y}_{m}$ as a function of $z / r_{\varepsilon}$ are included in table 5.

4.2.2 Unmixedness Measurements Along the Centerlines of Variable Denstty Jets

The rms values for the concentration fluctuations along the centerlines of the six jets discussed in section 4.2 .1 were calculated in terms of mole and mass fractions. The results of these calculations are reported in terms of unmixedness values defined as $\mathrm{X}_{\mathrm{m}}^{\prime} / \overline{\mathrm{X}}_{\mathrm{m}}$ and $\mathrm{Y}_{\mathrm{m}}^{\prime} / \overline{\mathrm{Y}}_{\mathrm{m}}$.

Values of $\mathrm{X}_{\mathrm{m}}^{\prime} / \overline{\mathrm{X}}_{\mathrm{m}}$ are plotted as functions of $z / \mathrm{r}_{0}$ in $\mathrm{flg} \cdot 26$. All of the variable density flows have an inftally rapid rise in unmixedness with downstream distance and then tend to change rather slowly as $z / r_{0}$ is increased 
further. There is a definite ordering of values of $\mathrm{X}_{\mathrm{m}}^{\prime} / \overline{\mathrm{X}}_{\mathrm{m}}$ at a given value of $z / r_{0}$ with $R_{\rho}$ value. Flows with larger $R_{\rho}$ values have higher $X_{m}^{\prime} / \bar{x}_{m}$ values than those with smaller jet to coflow density ratio. Another trend in the results is evident from fig. 26. After the initial rapid increase in the unmixedness, jets with $R_{\rho}<1$ have values of $\mathrm{X}_{\mathrm{m}}^{\prime} / \overline{\mathrm{X}}_{\mathrm{m}}$ which continue to increase slowly as the measurement volume moves further downstream. The opposite is true for jets with $\mathrm{R}_{\rho}>1$. Interestingly, the data for $\mathrm{R}_{\rho}=1\left(\mathrm{C}_{3} \mathrm{H}_{8} / \mathrm{CO}_{2}\right.$ flow) reach a value of $\mathrm{X}_{\mathrm{m}}^{\prime} / \overline{\mathrm{X}}_{\mathrm{m}} * 0.23$ between $z / \mathrm{r}_{0}=10$ and 20 and then remain essentially constant to the furthermost downstream position measured.

The appearance of the plot for unmixedness as a function of $z / r_{0}$ changes dramatically when mass fraction results are used. Figure 27 shows exper1mental data for the $s 1 x$ jets plotted in terms of $Y_{m}^{\prime} / \bar{Y}_{m}$. All of the experimental data now seem to be approaching a common asymptote of $\approx 0.23$. The downstream distance required for a given jet to reach this asymptote depends strongly on $R_{\rho}$. The he/air jet has reached an asymptotic value at $z / r_{0}=20$ while the $\mathrm{SF}_{6} /$ alr jet has not attalned such a value at $z / \mathrm{I}_{0}=63$. Jets having intermediate values of $R_{\rho}$ lie between these two extremes.

A close examination of the data for the He/air jet shown in f1g. 27 reveals that values of $Y_{m}^{\prime} / \bar{Y}_{m}$ first appear to reach an asymptote and then decrease slightly downstream. This behavior is only observed for the He/alr jet and is taken as further evidence for a wall effect on this flow. This conclusion is supported by the comparison of unmixedness measurements for the free and enclosed He/air jets shown in fig. 28. At lower values of $z / r_{0}$, the results for both jets lie close together. However, as $2 / r_{0}$ increases, they diverge somewhat. The free jet has an asymptotic value of $Y_{m}^{\prime} / \bar{Y}_{m}$ which $1 \mathrm{~s}$ similar to those for the other jets shown in fig. 27. 
It has been shown using approximate theoretical developments and by the experimental results in section 4.2 .1 that the similarity parameter $z / r_{\varepsilon}$ is more suitable for plotting variable density data of jet average centerline concentration than is $z / r_{0}$. We are aware of no such treatment which gives the proper similarity parameter for the behavior of centerline concentration intensity. Despite this, it might be assumed that $z / r_{\varepsilon}$ is also more suitable for treating intensity data. Figure 29 shows the unmixedness results for the six jets replotted as functions of $z / r_{\varepsilon^{*}}$ The resulting figure has some spread in the data, but there is a remarkable collapse of experimental results onto a common curve. Note that no virtual origins are included in this plot. If the virtual origins for the concentration intensity behavior have a similar dependence on $R_{\rho}$ as values of the average concentration, the collapse of the data in fig. 29 would be improved markedly.

4.2.3 Density Effects on Skewness and Kurtosis Values Along the Jet Centerline

Figures 30 and 31 show the results of measurements of $S$ and $K$, respectively, as a function of $z / r_{0}$ for the six gas pairs. At positions close to the nozzle $\left(z / r_{0}<20\right)$ there are large variations in the values of $S$ and K. For this reason, only values for $z / r_{0}>20$ are plotted. $S$ and $k$ results in terms of either mole or mass fractions are found to have very nearly the same values. This equivalence occurs because the third and fourth moments of the concentration fluctuations are normalized by the appropriate values of the rms to give $S$ and $K$. Only the values in mole fraction terms are shown.

There are some clear trends evident in the results for the centerline dependence of $S$ on $R_{\rho}$. For the jet with $\rho_{0}=\rho_{\infty}\left(\mathrm{C}_{3} \mathrm{H}_{8} / \mathrm{CO}_{2}\right)$ the values of $S$ 
appear nearly constant $(S \approx-0.26)$ over the range $20<z / r_{0}<65$. All of the different jet/coflow combinations seem to approach this same value of $S$ with increasing $z / r_{0^{\circ}}$. However, jets with $R_{\rho}<1$ have values of $S$ which increase with downstream distance while jets with $\mathrm{R}_{p}>1$ have values which fall. For all downstream distances there is a clear ordering of $S$ values with $R_{\rho}$, the He/air flow has the most negative values of $S$ and the $S_{6} /$ air jet the most positive. Note that the values of $S$ for the $\mathrm{CF}_{4} /$ air and $\mathrm{SF}_{6} / \mathrm{air}$ flows are initially positive, but decrease to negative values as $z / r_{0}$ is increased.

A similar, but not nearly as pronounced behavior, is found for values of kurtosis as a function of downstream distance. Figure 31 shows the results of the experimental measurements. By the time the flow fields reach $z / r_{0}=63$, values of $\mathrm{K}$ for all of the gas combinations are approaching $S=3$, the value for a Gaussian distribution. There is a dependence on $R_{\rho}$ such that jets having smaller values of $R_{\rho}$ have larger values of $K$. The two jets w1th $R_{\rho}$ less than one have the largest deviations from $K=3$. $K$ values for these jets decrease with increasing $z / r_{0}$. The negatively buoyant jets have only minor departures from $K=3$ throughout the flow region shown. There are apparently only minor increases in $\mathrm{K}$ for these jets as the observation volume is translated from $z / r_{0}=20$ to 65

\subsubsection{Reynolds Number Effects on Centerline Concentration Ealloff}

Table 2 lists the jets for which variable Re measurements have been made. These include three different Re measurements for the $\mathrm{C}_{3} \mathrm{H}_{8} / \mathrm{a} 1 \mathrm{r}$ and $\mathrm{SF}_{6}$ /air flows and two for the $\mathrm{CF}_{4}$ /air case. Only the two higher Re cases w11l be considered here for the $\mathrm{SF}_{6} /$ air jet since, as already discussed, the ning 
behavior for $\operatorname{Re}=3950$ is strongly modified by the strong negative buoyancy present for this jet.

Figures 32-34 show the centerline concentration behaviors for each of the variable Re flows. As before, the data is plotted as $Y_{0} / \bar{Y}_{m}$ versus $z / r_{0}$. The solid lines are results for linear least squares fits of the various sets of experimental data. Results of these fits are summarized in table 5 where values of the slopes $\left(K_{y}\right)$ and virtual origins $\left(z_{0}\right)_{y}$ are listed for each flow. For the $\mathrm{C}_{3} \mathrm{H}_{8} /$ air flow at $\mathrm{Re}=11,860$, the three data points at the lowest $z / \mathrm{r}_{0}$ values shown have not been used in the least squares fit.

For the $\mathrm{C}_{3} \mathrm{H}_{8} /$ air and $\mathrm{CF}_{4} /$ air jets the principal effect of increasing the Re is a downstream shift in the location of the virtual origin. There are only relatively minor variations in the slopes. Figure 35 shows the values of the virtual origins plotted as a function of Re for these two jets. The data are too limited to allow definite conclusions, but it appears that both flows have the same dependence of $\left(z_{0}\right)_{y}$ on $\operatorname{Re}$.

The $\mathrm{SF}_{6} / \mathrm{alr}$ data for $\mathrm{Re}=7890$ and 11860 shown in f1g. 34 lie very close together. Linear least squares fits of the data give IInes having slightly different values of $\left(K_{y}\right)$ and $\left(z_{0}\right)_{y}$. It is not surprising that the observed Re dependence for this jet/coflow combination differs from those found for the two gas pairs with lower values of $R_{\rho}$. As shown in section 4.2 .2 , the $\mathrm{SF}_{6} / \mathrm{air}$ flow approaches an asymptotic value of $\mathrm{Y}_{\mathrm{m}}^{\prime} / \mathrm{Y}_{\mathrm{m}}$ very slowly which indicates this flow is still developing over the range of $z / r_{0}$ values where measurements have been made. 


\subsubsection{Reynolds Number Effects on Centerline Unmixedness Behavior}

Values of $Y_{m}^{\prime} / \vec{Y}_{m}$ as a function of $z / r_{0}$ for the jet/coflow combinations discussed in the last section are shown in figures 36-38. Once again, the $\mathrm{C}_{3} \mathrm{H}_{8}$ /alr and $\mathrm{CF}_{4}$ /alr jets seem to have similar dependencies on Re. For the lowest Re studied, both jets have unmixedness values whfch attain an asymptotic value well before the end of the flow region over which measurements are made. In each case the value is $\approx 0.23$. As the $R e$ is 1ncreased, the growth of the concentration intensity along the centerline is altered markedly. After initial rapid rises in unmixedness values with downstream distance, the growth slows down and slow increases continue until the asymptotic value is attained considerably further downstream. Figure 36 shows that the varfations in the centerline development behavior of unmixedness with Re are largest when the $R e$ are small, but that variations are observed over the entire range of $\operatorname{Re}$ investigated.

The Re dependence of the centerline development of concentration intensity for the $\mathrm{SF}_{6} / \mathrm{a}$ ir flows is shown in $\mathrm{fig} .38$. As was the case for the centerline average concentration, there are only small differences between the measurements for $R e=7890$ and $R e=11860$. As already noted in sect1on 4.2.2, the values of unmixedness of this jet/coflow gas combination grow slowly along the jet centerline. For $z / r_{0}=63$, the values of $Y_{m}^{\prime} / \bar{Y}_{m}$ have only increased to $=0.19$. The experimental results for the two Re lie very close together. If a trend is present, it is that the lower Re jet develops slightly more rapidly with downstream distance than that for $\operatorname{Re}=11860$. This cunclusion $1 \mathrm{~s}$ the same as that for the two flows with smaller $R_{\rho}$ values. 
4.2.6 Reynolds Number Effects on Centerline Skewness and Kurtosis Values

The higher moments for the concentration fluctuations of the variable $\operatorname{Re}$ flows have been utilized to generate values of $S$ and $K$ along the centerlines of these flows. Increasing the Re results in no measureable variations of $S$ or $\mathrm{K}$ from those recorded at lower $R e$ and shown in figs. 30 and 31 , respectively.

\section{DISCUSSION}

The two measurement techniques utilized in this work provide very different types of information concerning the behavior of turbulent flows. The shadowgraph experiment is sensitive to integrated variations of the index of refraction and, at best, gives a qualitative view of the flow field. On the other hand, the Rayleigh light scattering technique provides quantitative measurements of concentration, but results are only reported for the concentration behavior along the centerlines of the jets studied. Despite the very different nature of the two experiments, the measurements are found to complement each other and, when taken together, provide a clear indication of the effects of global density differences on turbulent mixing.

In this section the experimental findings of this study are first summarized for both the shadowgraph and concentration results. Centerline concentration measurements are then compared with predictions based on Integral treatments of the equations for conservation of momentum and jet fluid given in section 2.2. Experimental findings of similar studies available in the literature are then discussed. A simple theoretical framework is 
proposed which provides qualitative predictions concerning the effects of jet/coflow density ratio and $R e$ on centerline average concentration fall of $f$ and unmixedness behavior. The last part of this section considers the question of whether or not the flows investigated here can be considered to be representative of free, purely momentum-driven, turbulent, axisymmetric jets.

\subsection{Summary of Experimental Findings}

\subsubsection{Effects of Global Density Differences on Turbulent Mixing}

That global density variations have an effect on turbulent mixing is evident from an examination of the shadowgraphs shown in figs. 5-19. These photographs indicate quite clearly that, for jets having the same Re, as the value of $R_{\rho}$ increases the initial radial growth rate of the jet as a function of $z / r_{0}$ is slowed. This is also evident from the variations in visually estimated values of $D$ for the flow at $z / r_{0}=40$ listed in table 3 .

Perhaps the most dramatic manifestation of density effects on the mixing behavior of jet flows are the fountains observed for the slow Re flows of $\mathrm{SF}_{6} / \mathrm{air}$ and $\mathrm{SF}_{6} / \mathrm{He}$. Figures 12-14 and 17-19 show examples of shadowgraphs recorded for these two combinations of jet and coflow gases. The concentration measurements shown in fig. 21 indicate that the tops of the fountalns for both of these jets occur at $z / r_{0} \approx 35$. These downstream distances agree very well with the location of the tops of the fountains indicated by the shadowgraphs shown in figs. 14 and 19. 
As suggested by the shadowgraphs of figs. 12 and 17 , concentration measurements made near the nozzle, but well outside of the jet, indicate the presence of $\mathrm{SF}_{6}$ in the coflow. It is clear that the general characteristics of these fountain flows, which can be inferred from their shadowgraphs, are consistent with the more exact concentration measurements.

In section 4.2 .1 it was noted that plots of $Y_{0} / \bar{Y}_{m}$ for different gas pairs as functions of $z / r_{\varepsilon}$ are expected to give straight lines having a common slope. Figure 25 shows the experimental data for six jet/coflow combinations plotted in this manner. It is clear that this data treatment gives a partial collapse of the experimental data. Values of the slopes, $K_{c}$, which result from least squares fits of the data are included in table 5. Both fig. 25 and the $K_{c}$ values show that there is a systematic variation in $K_{c}$ values with $R_{\rho}$.

The practical effect of plotting the experimental data in terms of $z / r_{\varepsilon}$ instead of $z / r_{0}$ is to multiply the slopes of the fits in terms of $z / r_{0}\left(K_{y}\right)$ by $R_{\rho}^{1 / 2}$ in order to give $K_{c}$. Comparison of $K_{c}$ values which result from measurements on the different jets indicates that improved fits are obtained if a higher power for $R_{\rho}$ is used than 0.5. Table 6 compares values of slopes which are found for plots of $Y_{0} / \bar{Y}_{m}$ versus $z /\left(r_{0} R_{\rho}^{0.6}\right)$. There is obviously a much improved correlation of the data compared to that found for the values of $\mathrm{K}_{\mathrm{c}}$ listed in table 5. It should be emphasized that an exponent of 0.6 is based purely on empirical observation. We are unaware of any theoretical development which would predict such a dependence on $R_{\rho}$.

Comparisons of the shadowgraphs and centerline measurements also yield consistent conclusions regarding the development and growth of turbulent 
fluctuations in these varlable density jets. The apparent lengthening of potential cores as visualized by shadowgraphy are well ccrrelated with the downstream distance required for the rapld rise of centerline unmixedness in mole fraction terms (see fig. 26). The distances required fir values of $\mathrm{x}_{\mathrm{m}}^{\prime} / \overrightarrow{\mathrm{x}}_{\mathrm{m}}$ to attaln a relatively slowly changing value increase as $R_{\rho}$ is increased. Similarly, the lengths of the potentlal cores in the shadowgraphs are observed to Increase as the value of $R_{\rho}$ becomes larger. This tendency for jets having larger $R_{\rho}$ values to require a longer downstream distance for the development of turbulence is also evident from a comparison of the downstream distances required by the various flows for Inftial vortex roll up which are listed in table 3 .

When the centerline unmixedness values are plotted in mass fraction terms, the importance of $R_{\rho}$ values to fet development becomes even more evident. All of the different jet/coflow combinations studied approach an asymptotic value of $\mathrm{Y}_{\mathrm{m}}^{\prime} / \overline{\mathrm{Y}}_{\mathrm{m}} \approx 0.23$ (see fig. 27). However, the downstream distances required for the varlous flows to approach this value are strongly dependent on the value of $R_{\rho}$. Jet/coflow combinations having $R_{\rho}<1$ are found to develop very rapidly and to only require a short downstream distance to attain an asymptotic value of $\mathrm{Y}_{\mathrm{m}}^{\prime} / \overline{\mathrm{Y}}_{\mathrm{m}} \cdot$ On the other hand, gas combinations which have $R_{\rho}$ much greater than 1 develop very slowly with downstream distance. In fact, at the largest downstream distance for which measurements have been made $\left(z / r_{0}=63\right)$, the $S F_{6} / a 1 r$ jet has not yet attained its asymptotic value of $\mathrm{Y}_{\mathrm{m}}^{\prime} / \overline{\mathrm{Y}}_{\mathrm{m}}$. Note that the results quoted here for the $\mathrm{SF} \mathrm{f}_{6} / \mathrm{a} 1 \mathrm{r}$ flow are for $\operatorname{Re}=7890$ while the other jet/coflow combinations shown in f1g. 27 are for $\operatorname{Re} \approx 3960$. As will be discussed shortly, higher Re are also found to be associated with longer flow development lengths. Desplte this, it is 
clear that the major source of the extremely slow development of this turbulent jet is its large value of $R_{\rho} \cdot$

Further evidence for the importance of density to development in turbulent flows appears in the plots of $\mathrm{Y}_{\mathrm{m}}^{\prime} / \overrightarrow{\mathrm{Y}}_{\mathrm{m}}$ for the different flows as functions of $z / r_{\varepsilon}$ shown in fig. 29. The use of the effective radius concept has been shown to allow a partial correlation of the average centerline concentration behavior for jets having global density differences from their surroundings. The same concept also appears to collapse data for the unmixedness behavior. It is interesting to note that if $R_{\rho}$ were taken to the 0.6 power as suggested by the average concentration results discussed above, there would also be an improved collapse of the unmixedness data.

The behaviors of $S$ and $K$ values which have been measured for the various jet/coflow combinations have clear dependencles on $R_{\rho}$ as described in section 4.2.3. However, it is difficult to correlate these measurements with those of average concentration or unmixedness behavior. The unmixedness measurements Indicate that the He/air flow develops asymptotic behavior at the shortest downstream distance of any of the flows studied here. It might be expected that this flow would also have small variations in $S$ and $K$ values. This is not the case. Figures 30 and 31 show that this particular flow has the largest variations in the values of $S$ and $K$. It may well be that the variations in the values of $S$ and $K$ are not only due to turbulent fluctuations, but may also be due to other processes such as molecular diffusion.

In section 4 it was noted that the mixing behavior for the He jet flowing into a coflow of air is believed to have been influenced by the presence of 
the enclosure to a much greater degree than the other gas combinations studied. There are no clear indications of such variations indicated by comparison of the shadowgraphs of the enclosed and free helium jets shown in figs. 5 and 6, respectively. Spreading rates with downstreal distance and turbulent structure appear to be the same in both cases. Nevertheless, comparison of these two shadowgraphs with those for the other jet/coflow gas combinations shows clearly that the He turbulent jet into air spreads radially much more rapidly as a function of downstream distance than do the other flows. For this reason, the He/air jet will interact with the enclosure walls at a much shorter downstream distance than the other gas flows.

To further complicate the problem, the flow volume of air for the enclosed He jet is limtted and is not high enough to insure that recirculation eddies will not develop. In fact, the calculated downstream distance for complete depletion of the coflow listed in table 2 is $z_{d} / r_{0}=56$, which 11 es within the range of downstream distances observed during the study. Such recirculation eddies will surely modify the mixing behavior along the jet centerline. This situation is exacerbated by the extremely rapid flow development observed for the He turbulent jet. This rapid development assures that the turbulent flow reaches its maximum entrainment capability after only a short distance downstream.

The behavior of the centerline mass fraction unmlxedness provides a clear Indication that modifications of mixing behavior for the He/alr flow occur due to wall and/or recirculation effects. Figure 27 shows that the He/a1r flow $1 \mathrm{~s}$ the only gas pair studied for which values of $\mathrm{Y}_{\mathrm{m}}^{\prime} / \overline{\mathrm{Y}}_{\mathrm{m}}$ reach a maxtmum and then decrease. Further evidence for the importance of these effects is found by 
comparing the measurements of average concentration and mass fraction unmixedness made for the enclosed He/air jet with similar results for a free He jet at an equivalent $\operatorname{Re}$ (see figs. 24 and 28 ). These results show that values of $Y_{o} / \vec{Y}_{m}$ and $Y_{m}^{\prime} / \bar{Y}_{m}$ for these two flows lie very close together at $z / r_{0}=31.5$, but then have an increasing divergence as the flow fields move further downstream. For both average concentration and unmixedness, the values for the enclosed jet fall below those of the free jet at equivalent downstream distances.

It is significant that the values of $\mathrm{Y}_{\mathrm{O}} / \overline{\mathrm{Y}}_{\mathrm{m}}$ for the He free jet form a straight line for values of $z / r_{0}$ extending to 110 . Values of $Y_{m}^{\prime} / \bar{Y}_{m}$ for the free jet (fig. 28) show some variation, which might be expected due to the sma11 number of concentration measurements (100) used in their calculation. The observed values of $\mathrm{Y}_{\mathrm{m}}^{\prime} / \vec{Y}_{\mathrm{m}}$ indicate that the downstream unmfxedness for the free He jet is roughly constant at $Y_{m}^{\prime} / \bar{Y}_{m} \approx 0.26$. This value is only slightly higher than that of 0.23 which is estimated for the jets flowing within the enclosure.

On the basis of the above discussion, we have chosen to use the free jet measurements of the average concentration when making comparisons with similar results for other jets. It has also been assumed that the drop in unmixedness values observed for the enclosed He/air flow at downstream distances is an artifact and that if recirculation and wall effects were absent, observed values of $Y_{m}^{\prime} / \bar{Y}_{m}$ would remain constant as $z / r_{0}$ is increased. 


\subsubsection{Ef fects of Reynolds Number Variations on Turbulent Mixing}

The majority of measurements which have been performed during the course of this study were made for $\operatorname{Re} \approx 3960$. The use of such low Re serves to limit the volumes of gas flows and at the same time are more compatible with the simple flow system used. Unfortunately, turbulence researchers often question whether axisymmetric turbulent jets operating at such low Re are fully turbulent. For this reason, a limited study of Re effects on the turbulent mixing behavior of the $\mathrm{C}_{3} \mathrm{H}_{8} /$ air, $\mathrm{CF}_{4}$ /air and $\mathrm{SF}_{6}$ /air flows has been made.

Shadowgraphs of the three $\mathrm{C}_{3} \mathrm{H}_{8} /$ air (figs. 9-11) and the three $\mathrm{SF}_{6} /$ air (figs. 12-16) variable Re flows are described in sections 4.1 .4 and 4.1.5, respectively. Similar variations in the appearance of the shadowgraphs are observed for each gas pair as the Re is increased. With the exception of the lowest $\operatorname{Re} \mathrm{SF}_{6} /$ air case, the spreading rate of the jet for a particular gas combination seems to be independent of $\mathrm{Re}$. On the other hand, the turbulent structures observed within the turbulent flow fields of the jets become more intense and have smaller scales as the $R e$ is increased. The development of turbulence near the nozzle is also modified by changes in the flow Re. Decreasing potential core lengths occur for increasing Re. Simliar reductions In the distance from the nozzle to Initial vortex roll up with increasing Re can be seen in table 3 .

The concentration measurements along the centerline produce a different view of Re effects on the turbulent mixing behavior. These experiments show that increasing $R e$ result in downstream shifts in the positions of the virtual origins which are derived from plots of $Y_{0} / \bar{Y}_{m}$ versus $z / r_{0}$ (see $\mathrm{f} i g \cdot 35$ and 
table 5), but that the values of $K_{y}$ remaln essentially constant. Values of unmixedness as a function of downstream distance still approach a common asymptote, but as the Re becomes larger the flow distance required to achieve asymptotic behavior is increased dramatically.

Measurements made for constant Re have indicated that increasing the value of $R_{\rho}$ leads to a slower development of the fet which is reflected in slower initial development of the turbulence near the fet nozzle as well as a slower approach to an asymptotic value of $\mathrm{Y}_{\mathrm{m}}^{\prime} / \overline{\mathrm{Y}}_{\mathrm{m}} \cdot$ Holding $R_{p}$ constant and Increasing the Re leads to a different turbulence behavior. Increasing the $\operatorname{Re}$ results in a more rapid initial development of the fet near the nozzle as reflected in the observed shorter potential core lengths. At the same time, the growth of the jet towards an asymptotic unmixedness value is slowed and the locations of the virtual origins for plots of the centerline concentration data are shifted downstream.

\subsection{Comparison of Average Centerline Behavior with Integral Analysis Predictions}

The results of several approximate solutions of the integral equations for variable density turbulent flows are given in sections 2.2 and 2.3 . In this section the experimental findings of this study w111 be compared with predictions of mixing behavior based on these solutions.

Perhaps the most important conclusion that can be derived from this type of analysis is that plots of $\mathrm{Y}_{\mathrm{O}} / \bar{Y}_{\mathrm{m}}$ versus $z / \mathrm{r}_{\varepsilon}$ for data recorded at sufficlently large downstream distances should have linear behaviors with slopes which are Independent of $R_{\rho}$. It has already been shown that the use of the 
effective radius concept, where $r_{\varepsilon}$ is defined by eq. (22) with $\rho_{f}=\rho_{\infty}$, gives a partial correlation of the centerline concentration falloff data for the variable density jet/coflow combinations investigated. However, it has also been shown that such a correlation is improved markedly if an exponent of 0.6 is employed for $R_{\rho}$ instead of that of 0.5 derived from integral analysis.

Deviations from the predicted dependence of $K_{c}$ on $R_{\rho}$ may be a characteristic of free jets or it may be due to one or all of several differences which exist between the flows studied here and truly free, momentum-driven variable density jets at far downstream distances. It must be remembered that the integral analysis used to derive the dependence of the centerline concentration falloff on $R_{\rho}$ assumes that the centerline density and the density of the surrounding gas are very nearly the same. The measurements reported in this study have been made at relatively short downstream distances, $20<z / r_{0}<63$, for which this condition is not met. The ordering and relative magnitudes of the variations in the slopes of the linear least squares fits of the data to eq. (24) are consistent with such an interpretation.

The use of a coflowing surrounding gas may also modify the observed slopes. Table 2 shows that as the primary flow to secondary flow density ratio increases from that for $\mathrm{He} / \mathrm{air}$ to that for $\mathrm{SF}_{6} / \mathrm{He}$, the relative velocity ratio of the coflow and jet, $m=U_{\infty} / U_{0}$, increases. In section 2.31 was pointed out that increasing values of m result in less rapid spreading rates for a turbulent jet with increasing downstream distance and to a less rapid falloff of centerline concentration. Such a modification from free jet behavior is consistent with the observed variations of $K_{C}$ with changes in $R_{\rho}$. However, it must be remembered that m values are relatively small even in case of the $\mathrm{SF}_{6} /$ air flow. 
The predicted dependence of $K_{c}$ on $R_{\rho}$ was derived assuming uniform profiles at the exit of the jet nozzle. It is possible that the exit profiles actually vary somewhat for the different gas flows. If this is the case, the values of $M_{0}$ will also vary and the slopes of the least squares fits will not have the expected dependence on $\mathrm{R}_{\rho} \cdot$

Equation (34) has been derived in section 2.2 using an analysis similar to one given by Thring and Newby [18]. This equation predicts a variation in the locations of virtual origins with $R_{\rho}$ for mass fraction data plotted in the form of eq. (24). By choosing an appropriate constant value for the term $\mathrm{K}_{122} / 2 \mathrm{~K}_{22}-\mathrm{K}_{122} / \mathrm{K}_{12}$ (here -0.5 ) and setting $\mathrm{K}_{12} / \mathrm{K}_{2}^{1} \mathrm{~L}^{2}=0.114$, eq. (34) leads to the predictions of $1 / \bar{C}_{m}$ falloff as a function of $z / r_{0}$ shown in $f i g \cdot 39$ for the values of $R_{\rho}$ used in the experimental study. Comparison of $f i g s \cdot 23$ and 39 shows that there is good qualitative agreement between the experimental findings and the calculated variations of $\mathrm{Y}_{\mathrm{O}} / \bar{Y}_{\mathrm{m}}$ with $z / \mathrm{r}_{\mathrm{O}}$. Of course, quantitative agreement is not found, since, as we have already noted, the use of the effective radius concept does not yield a complete collapse of slopes for the experimental data. On the other hand, the magnitudes and signs of the virtual origins are predicted quite well by this approximate treatment of the integral equations.

The analysis suggested by Rodi [5,23], which is discussed in section 2.2, can also be used to generate predicted behaviors for $\bar{C}_{m}$ as a function of $z / r_{0}$ for variable density flows. The results of such calculations for the values of $R_{\rho}$ employed are shown in $\mathrm{fig} .40$ where values of $\bar{C}_{m}$ are plotted as functions of $z / r_{0}$. These calculations require the input of an emplrical value for the ratio of the concentration to velocity radial half widths, $R$ (see eq. 
40). The value recommended by Chen and $\operatorname{Rod} 1$ [5], $R=1.17$, has been used. Note that the analysis assumes that $R$ is constant. throughout the flow field.

Figure 41 shows the results of linear least squares f1ts of $1 / \vec{C}$ versus $z / r_{0}$ for the results of $f 1 g \cdot 40$. The $f 1 t$ for $R_{\rho}=1$ gives a slope of $K_{C}=0.92$. This value is smaller than that of $K_{C}=0.114$ which has been measured experimentally in this study. For this reason, the calculated curves for the different flows are in poor quantitative agreement with experimental results. Increasing the value of $R$ leads to a higher slope, but a systematic varfation has not been attempted and only the results for $R=1.17$ are reported.

The lines shown in fig. 41 are found to collapse to a single line if the calculated values of $1 / \bar{C}_{m}$ are plotted as functions of $z / r_{\varepsilon}$. The analysis of Rodl therefore $g$ Ives the same dependence of $K_{c}$ on $R_{\rho}$ as that deduced from Thring and Newby [18]. This is not too surprising since both analyses use the conservation equations for momentum and jet flufd.

Rodi's formulation seems promising as a useful means for predicting jet centerline concentration behavior. It will be interesting to apply it to a set of experfmental data where velocity and concentration measurements have both been made throughout the flow fleld. Such measurements w11l allow a much better evaluation of whether or not the assumption of a constant value for $R$ Is correct and also allow an experimental determination of 1 ts value.

An Integral analysis is given In section 2.3 which generates an approx:mate expression for the height of the fountain $\left(z_{\max } / r_{0}\right)$ formed by a negative- 
ly buoyant jet. These heights have been calculated using eq. (58) for the flows studied which have values of $R_{\rho}$ greater than one. The value of the jet spreading angle required for use in eq. (58) was chosen to be that recommended by Chen and $\operatorname{Rod} 1[5]\left(\theta=6.3^{\circ}\right)$. The empirical expression of Turner [24], eq. (59), has also been used to calculate $z_{\max }$. Results for both expressions are summarized in table 7 .

Both equations predict values of $z_{\max }$ which are in good agreement with the estimates of $z_{\max } / r_{0}=38$ for the low Re $S F_{6} /$ air and $z_{\max } / r_{0}=44$ for the $\mathrm{SF}_{6} / \mathrm{He}$ flows from the shadowgraphs. As expected, the other flows have values of $z_{\max }$ which lie outside of the range of experimentally observed values. The experimental results are too limited to allow a determination of which equation $((58)$ or $(59))$ describes the density dependence of $z_{\text {max }}$ most accurately.

\subsection{Comparison of Experimental Results with Past Work}

Experimental analysis of the concentration fields of axisymetric turbulent jets has historically been and remains the focus of a great deal of interest in the literature. In this section the results of the current study will be compared with and discussed in relation to this earlier work.

Concentration measurements in turbulent axisymmetric jets fall into two general classes--cases where the jet and the surrounding gas have the same density and cases where these densities vary ( $1 . e ., \rho_{0} \neq \rho_{\infty}$ ). Constant density flows (usually afr jets into air) are marked in some manner in order to allow concentration variations to be followed. Markers have included small 
particles, minor temperature variations, and trace concentrations of a second gas. In cases where temperature or chemical markers are utilized, the variations in density are small enough to assume constant density mixing. Variable density cases have been studied for flows having variable temperature, velocity (compressibility effects), and chemical composition. Density variations due to compressibility will not be included in this discussion.

\subsubsection{Average Centerline Concentration Measurements}

There are a large number of experiments which have measured average jet fluid concentration along the centerlines of axisymmetric jets. Table 8 summarizes the results of many of these studies along with parameters for the various flows. The centerline concentration falloff is characterized in terms of eq. (24) where $\bar{C}_{m}$, as defined by eq. (25), can represent either chemlcal composition or temperature. Values of $K_{c}$ and $\left(z_{0}\right)_{c}$ are listed where available. In some studies, marked with an *, these values have been generated using results taken from figures in the original papers. It has also been necessary for a few of these data sets to convert from mole fraction measurements to mass fraction results. No corrections for turbulent fluctuations were utilized in these conversions. Resulting values of $\bar{Y}_{m}$ are expected to be accurate to within a few percent [10].

There are several experimental investigations which have not been Included in table 8 because their results are not In formats which allow values of $K_{c}$ and $\left(z_{0}\right)_{c}$ to be readily calculated. Among these are studies by Keagy and Ellis [43], Uberol and Garly [44], and Shaugnessy and Morton [45]. Keagy and Ellis [43] utillzed a quantitative Schlieren method to measure the 
falloff of centerline concentration for a He jet into air. The Re for the flow was not reported, but was apparently quite high. For results plotted as $1 / \vec{X}_{\mathrm{m}}$ versus $z / \mathrm{r}_{0}$ they find a straight line having a slope of $\mathrm{K}_{\mathrm{x}}=0.053$. This value is in good agreement with that found for the same gas pair in this study, $\mathrm{K}_{\mathrm{x}}=0.048$ (see table 5). Uberoi and Garby [44] have investigated flows of cooled air into room air. Jets having initial temperatures as low as $100 \mathrm{~K}$ were used. The experimental results are sparse, but they do indicate that the normalized average temperature, expressed in the form of $\bar{C}_{\mathrm{m}}$, falls off much more slowly along the centerline for the cooler (more dense) jets. This finding is consistent with the results of this work. Shaughnessy and Morton [45] have investigated mixing in high Re flows of air into air using nephelometry. Radial profiles are presented at several downstream positions. Unfortunately, the centerline behavior is never explicitly given.

The measurements summarized in table 8 have been made using a wide range of experimental techniques and for various flow velocities, nozzle diameters, and nozzle shapes. For this reason, the initial jet velocity profiles and turbulence levels are expected to vary markedly from study to study. Given these variations in measurement techniques and initial flow conditions, values of $K_{c}$ for the different studies summarized in table 8 agree to a remarkable degree. An average of all of the $K_{c}$ values listed in the table yields $\mathrm{K}_{\mathrm{c}}=0.105$ with an average deviation of $\pm 10 \% . \mathrm{K}_{\mathrm{c}}$ values for the variable density flows investigated in this study (table 5) lie well within this range of values.

It is very difficult to discern any dependence of $K_{c}$ on $R_{\rho}$ or $\operatorname{Re}$ from the results listed in table 8 . Even though the variations in the values of $K_{c}$ are 
relatively small from study to study, they are large enough to mask small dependencies on these parameters. If any trend is evident in the data, it is that the jets having the largest Re seem to have lower values of $K_{c}$. It must be emphasized that this is a highly tentative conclusion based on the results summarized in table 8 . Such a dependence has not been identfied in the limited investigation of Re effects in the current study.

There are very few studies where variable density flows have been systematically investigated. From the results listed in table 8 it is impossible to verify whether $K_{c}$ has the dependence on $R_{\rho}$ which has been deduced from this study. The value of $\mathrm{K}_{\mathrm{c}}$ listed for the results of W1lson and Danckwerts [20] is actually a fit of several sets of data covering a range of $R_{\rho}$ values from 1.04 to 1.68. The concept of the effective radius [18] was used for the fit, but no allowance was made for shifts in virtual origins and a value of 0 was assumed for each set of results. This study [20] supports the validity of Thring and Newby's concept, but the data are not recorded over a wide enough range of $R_{\rho}$ to determine an accurate value of the exponent for $R_{\rho}$. Because of the treatment of the data, any dependence of $\left(z_{o}\right)_{c}$ on $R_{\rho} 1 s$ masked.

Scheffer and Dibble [42] have reported a detalled study of a $\mathrm{C}_{3} \mathrm{H}_{8} / \mathrm{alr}$ flow. In this work some measurements of a $\mathrm{CH}_{4} / \mathrm{alr}$ flow were also made. When both sets of data are plotted in the form of eq. (24), the $\mathrm{CH}_{4} / \mathrm{air}$ measurements lie above those for the $\mathrm{C}_{3} \mathrm{H}_{8}$ /air flow. This experimental result for their high Re flows is consistent with a stronger dependence of the effective radius on $R_{\rho}$ than 1ndicated by the half power included in eq. (22). 
It is 1mpossible to reach any conclusions based on the tabulated results concerning the dependence of $\left(z_{0}\right)_{c}$ on $R_{p}$ or Re. The clear dependence of $\left(z_{0}\right)_{c}$ on $R_{\rho}$ identified in this study will be masked by Re varlations and the different inftial conditions among the summarized investigations. Simflarly, varlations in $\left(z_{0}\right)_{c}$ with Re seen in this investigation (see table 5 and fig. 35) cannot be discerned from results listed 1n table 8. Ebrahimi and Kleine [15] do Indicate that values of the axial concentration half lengths shift downstream with increasing $\operatorname{Re}$ (see eq. (15) and discussion in section 2.1). This Is the only evidence of which we are aware that values of $\left(z_{0}\right)_{c}$ increase with increasing Re. However, as discussed in section 2.1 , similar shifts with Re have been identified in velocity measurements.

It must be concluded that the data avallable for average jet fluid concentration along the centerlines of axisymetric jets all yield roughly the same value for slopes of data plotted as $1 / \bar{C}_{m}$ versus $z / r_{\varepsilon}$ and that there may be a very weak dependence of $K_{c}$ values on $R_{\rho}$. However, the data are not sufficlently reproducible from experiment to experiment to confirm the relatively small effects of $R_{\rho}$ on $K_{c}$ values or the shifts 1 n values of $\left(z_{0}\right)_{c}$ with

changes in $R_{\rho}$ or $R e$ which have been characterized in this study. The use of a single flow system and flow dlagnostic for a number of jet/coflow gas pairs covering a wide range of density ratios is the key to the identification of these trends in the current work.

\subsubsection{Unmixedness Centerl1ne Measurements}

The measurement of unmixedness in turbulent flows is considerably more difficult than the determination of average concentration values. For this 
reason, until recently the number of unmixedness measurements has been very limited. However, the development of new diagnostics in the past few years has led to many such measurements. As will be shown, findings among different studies have not agreed well.

Table 9 lists various literature sources for centerline unmixedness measurements of jet fluid in turbulent jets. Included are tracer measurements in constant density flows as well as variable density flows having varying temperature or chemical composition.

In the past, many authors $[10,15,16,39,41,48,50]$ have shown plots which compare their centerline unmixedness measurements with those of several different workers. Such plots are extremely useful for comparing different sets of data and do give a good indication of the wide variation in concentration intensity behavior which has been observed. Unfortunately, the number of studies available is becoming so large that several plots would be required to compare our results for centerline unmixedness shown in figs. $27-29$ and $36-38$ with available literature findings. Instead, the various studies are compared in table 9 by listing some general characteristics for each flow and some parameters for the behavior of centerline unmixedness.

All of the studies discussed have found that there is a rapld increase in unmixedness with downstream distance beyond the potential core region. After this rapid increase, the unmixedness begins to level off. At downstream distances beyond the region of rapid rise, two different behaviors for unmixedness growth have been reported. Some workers find that the values of $\mathrm{C}_{\mathrm{m}}^{\prime} / \bar{C}_{\mathrm{m}}$ attain an asymptotic value while others claim that no such asymptote 
is reached and unmixedness values continue to increase slowly over the entire range of $z / r_{0}$ values investigated. Table 9 lists whether or not asymptotic values are achieved and, if so, the approximate downstream location where asymptotic behavior is first reached. Values of the maximum unmixedness observed along the centerline are also included in the table. For those jets which have an asymptotic behavior this value is the observed asymptote while It is simply the largest value of $C_{m}^{\prime} / \bar{C}_{m}$ observed for jets which do not. The final column of table 9 lists the maximum downstream distance in jet radif for which measurements have been reported.

A few notes concerning the studies listed in table 9 are appropriate. Corrsin and Uberof [6] report unmixedness values for a heated jet which reach a maximum near the nozzle and then fall off with downstream distance. This is the only study listed in table 9 which has such a behavior and is most likely due to an experimental artifact. Some of these studies $[9,15,37]$ have shown maxima near the potential core region of the flow field followed by development of unmixedness much like that observed in this and other studies. We feel that these observations may also be due to experimental artifacts. The most likely source is a lack of sufficient spatial resolution for measurements taken near the jet nozzle where the unmixedness is changing rapidly with downstream distance.

Values for the maximum $C_{m}^{\prime} / \bar{C}_{m}$ observed generally fall in the range 0.18 to 0.25. Measurements using hot-wire anemometry techniques by Way and Libby [47] and McQuaid and Wright [37] lie well outside of this range. It may be that the hot-wire measurement technique is not able to compensate properly for the high turbulence levels found in this type of jet and the high readings 
observed are the result. Excluding these two studies, there is still a wide varlation in measured results. It would be highly speculative on our part to comment on possible sources for these variations. There is no reason to suspect that one experimental technique is superior to another. On the other hand, it is difficult to accept that such large variations in $C_{m}^{\prime} / \bar{C}_{m}$ are Indicative of the actual behavior of these flows. Clearly, more extensive studies w11l be necessary to unravel the true source of these var1ations.

It is also perplexing that some jets seem to attain asymptotic values of unmixedness while others do not. Some of these studies have extended measurements to quite large downstream distances. The data are not extensive enough to make definite statements, but as will be discussed shortly, most of the data are not inconsistent with the variations in centerline unmixedness with $R_{\rho}$ and $R e$ which have been identified in this study.

Table 10 summarizes the centerline unmixedness results of this study in the same manner as the 11terature results listed 1n table 9. As already noted in section 4.2 .2 based on plots of the results, the measurements show clearly that asymptot1c values of unmlxedness are attained for the low $\operatorname{Re}(-4000)$ jets. The downstream distance required for a given jet/coflow palr to reach an asymptotic value increases rapidly with $R_{\rho}$. HIgher $R_{e}$ also lead to slower centerline growth of $\mathrm{C}_{\mathrm{m}}^{\prime} / \overline{\mathrm{C}}_{\mathrm{m}}$ values.

Table 9 includes the results of an experiment on a $\mathrm{CH}_{4} /$ air flow which we have previously described $[1,2]$. Comparison with table 10 shows that different conclusions have been reached concerning the existence of an unmixedness asymptote desplte the fact that the two sets of data are in close quantitative 
agreement. The signal to noise levels in the earlier experiment were lower and the resulting measurements of unmixedness are more scattered than are the current results shown in fig. 27. Furthermore, a small misalignment of the jet centerline and the light scattering observation volume has been corrected since the original work was completed. These two differences are belleved to be sufficient to explain the conflicting conclusions for studies made in the same flow.

Most of the studies in table 9 which show an asymptotic behavior for unmixedness either have relatively low $R e$ or have values of $R_{\rho}<1$. This observation is consistent with the conclusions of this study concerning the dependence of jet centerline concentration fluctuation behavior on $R_{\rho}$ and Re. Some experiments clearly do not conform to these criteria. For instance, the flow conditions of the free $\mathrm{CH}_{4}$ /air flow of Birch et al. [10] Indicate that this jet should attain an asymptotic value of unmixedness. Their data indicate quite clearly a continual increase along the entire centerline length for which measurements were made. The data of Niwa et al. [41] for the He/air flow were taken for a jet having an extremely low Re and it is not clear that this jet will be turbulent. Two recent experiments on water jets $[16,48]$ at $\operatorname{Re}=5000$ are included in table 9 which give different conclusions concerning the existence of an unmixedness asymptote. Both of these studies utilized absorption measurements of dye solution for jet fluid determination. Dahm [16] used an innovative external line measurement technique while Nakamura et al. [49] employed an internal fiber optic probe. Given the similarity of the two flows it is difficult to understand the source of the different results of the two studies. 
Indirect evidence for the dependence of centerline unmixedness development on $R e$ and the existence of an asymptotic value of unmixedness is available in studies of jet entrainment. It is expected that centerline unmixedness will be correlated in some manner with the entrainment behavior of the jet. On this basis, one would predict that more surrounding fluld would be entrained into these flows near the nozzle for lower Re flows. Evidence for this can be found in the studies of Ricou and Spalding [21] and Hill [22]. Hill's local entrainment measurements show clearly that a finite downstream distance is required for a jet to attain an asymptotic entrainment rate. At positions near the nozzle, the jet was found to have a lower entrainment rate which grew with downstream distance. These findings suggest that there is indeed a correlation between the entrainment behavior and centerline unmixedness. Ricou and Spalding have shown that the entralnment of ambient fluid is dependent on Re for posttlons near the nozzle. Flows having Re on the order of $10^{4}$ entralned fluid much more efflclently than jets at higher Re. This observation is in agreement with the hypothesis, based on centerline unmixedness observations, that the entrainment capability for lower Re flows w1ll increase more rapidly as a function of downstream distance.

It has been pointed out by several groups $[1,2,9,10,15]$ In the past that when Inverse values of $C_{m}^{\prime}$ are plotted as a function of $z / r_{0}$ a linear growth $1 \mathrm{~s}$ found after the Initial jet development region. This growth behavior can be expressed mathematically with an expression simflar to eq. (12),

$$
\frac{C_{0}}{C_{m}^{\prime}}=K_{c}^{\prime} \frac{z-\left(z_{0}^{\prime}\right)_{c}}{r_{0}}
$$


where the primes indicate a slope $\left(K_{c}^{\prime}\right)$ and virtual origin $\left(z_{o}^{\prime}\right)$ for plots of inverse $\mathrm{C}_{\mathrm{m}}^{\prime}$. Dividing eq. (12) by eq. (69) yields an expression for unmixedness,

$$
\frac{C_{m}^{\prime}}{\bar{C}_{m}}=\frac{K_{c}}{K_{c}^{\prime}} \frac{z-\left(z_{o}\right)_{c}}{z-\left(z_{o}^{\prime}\right)_{c}}
$$

This equation predicts that at sufficiently large downstream distances the unmixedness will attain an asymptotic value of $\mathrm{K}_{c} / \mathrm{K}_{\mathrm{c}}^{\prime}$. The downstream distance required for an approach to this value is determined by the difference between the locations of the virtual origins for the average and rms plots. If $\left(z_{o}\right)_{c}$ is nearly the same as $\left(z_{0}^{\prime}\right)$, the jet will attain an asymptotic value of unmixedness at relatively short downstream distances. On the other hand, large differences in the values of virtual origins will insure that an unmixedness asymptote will occur only at large $z / r_{0^{\circ}}$ Equation (70) is useful because it treats the data in a manner which allows easy prediction of average and rms concentration values for a given flow at any value of $z / r_{0}$ beyond the potential core and also allows the approach of the jet toward an unmixedness asymptote to be predicted.

The existence of different virtual origins for plots of $C_{0} / \bar{C}_{m}$ and $C_{0} / C_{m}^{\prime}$ versus $z / r_{0}$ implies that these jets can not be truly fully developed over the axial downstream distances for which the least squares fits have been made. This requires further that $K_{c}$ and $K_{c}^{\prime}$ cannot be true constants. However, these values are found to vary so slowly with downstream distance that linear fits of the data remain quite accurate. For this reason, their use is appropriate. 


\subsubsection{Centerline Measurements of Skewness and Kurtosis}

Very few measurements of the higher moments of jet fluid concentration fluctuations along the centerlines of axisymmetric turbulent jets are avallable in the 1iterature. Ebrahimi et al [50] have measured both skewness and flatness values for a constant density air/air jet. Values of variable density flows have been previously described for $\mathrm{CH}_{4} /$ air $[1,2]$, natural gas/air [10], and heated afr/air [39] flows. The heated afr jet had a denstty ratio of $R_{\rho}=0.51$ which is very close to the value of 0.55 for the methane jet and 0.60 for the natural gas jet.

Figure 30 shows that the constant density $\mathrm{C}_{3} \mathrm{H}_{8} / \mathrm{CO}_{2}$ gas pair gives skewness values which are nearly constant over the range of $z / r_{0}$ shown. There 1 s a gradual drop from $S \approx-0.25$ at $z / r_{0}=20$ to $S \approx-0.30$ at $z / r_{0}=62$. Values of $\mathrm{K}$ (fig. 31) over the same range of downstream distances remain constant at the Gausstan value of 3 . The $S$ and $K$ results reported by Ebrahfm1 et al [50] show a very different behavior. Values of $S$ increase from $\approx-0.3$ at $z / r_{0}=20$ to $\approx 0.6$ at $z / r_{0}=100$. On the other hand, values of $K$ remain nearly constant at roughly 4.3 over the same range of $z / r_{0}$.

Our previous measurements of $S$ and $K[1,2]$ along the centerline of the $\mathrm{CH}_{4}$ /air jet are in excellent agreement with results shown in figs. 30 and 31. The value of $S$ for this turbulent jet $1 s=-0.6$ at $z / r_{0}=20$ and Increases with downstream position to -0.3 at $z / r_{0}=62$. Values of $k$ are found to be 3.4 at $z / r_{0}=20$ and to fall to $k \approx 3.0$ at $z / r_{0}=62$. B1rch et al [10] also find negative values of $\mathrm{S}$ which remain relatively constant at $S \approx-0.3$ over the range of downstream distances investigated wh1le Lockwood 
and Moneib [39] report a fall in $S$ values from $\approx-0.05$ to $\approx-0.3$ on going from $z / r_{0}=20$ to 100. The centerline behavior of $\mathrm{K}$ values measured by Birch et al are in excellent agreement with those shown in fig. 31. Again the results of Lockwood and Moneib differ slightly, showing a relatively constant value of $K \approx 2.9$ from $z / r_{0}=10$ to 100 .

Based on these limited comparisons, it is concluded that the results of Ebrahimi et al [50] are inconsistent with other published measurements and that our measurements of $S$ and $K$ are in good agreement with the other two experiments which have reported axial measurements of the higher moments for concentration fluctuations. There is no independent experimental verification for the dependencies of $S$ and $K$ on $R_{\rho}$ shown in figs. 30 and 31 , but, based on the agreement between experiments having $R_{\rho} \approx 0.55$, it is felt that the measurements for the other jet/coflow combinations provide a true indication of variations in $S$ and $K$ with $R_{\rho}$.

5.4 Centerline Falloff of Average Concentration and Growth of Unmixedness-Theoretical Considerations

It has been shown for the low Re jets investigated in this study that the average centerline concentrations and intensities are dependent on both $R_{\rho}$ and Re. Concentration data for constant Re jets plotted as $Y_{0} / \bar{Y}_{m}$ versus $z / r_{0}$ give straight lines with slopes which decrease and values of $\left(z_{0}\right)_{y}$ which move upstream as $R_{\rho}$ increases. If $R_{\rho}$ is fixed, increasing the $R e$ gives results which lie on lines having the same slope as at lower Re, but for which the virtual origins have moved downstream. Values of $Y_{m}^{\prime} / \bar{Y}_{m}$ initially increase with downstream distance and then attain an asymptotic value. The value of $\left(Y_{m}^{\prime} / \bar{Y}_{m}\right)_{\text {asym }}$ is invariant with $R_{\rho}$ or $R e$. Increasing values of either $R_{\rho}$ or 
Re increase the downstream distance required for unmixedness to reach this value. Comparisons with available literature results are inconclusive, but suggest that similar dependencies exist for axisymmetric jets of considerably higher Re. The obvious question is: Is there a theoretical framework with which to explain these empirical observations? In this section, a very simple analysis will be proposed which seems to provide such a qualitative framework. Further experimental and theoretical effort will be necessary to demonstrate whether this interpretation is correct and, if so, to quantify the predictions.

The "classical" picture of turbulence [51-54] is often discussed in terms of the eddies which are characteristic of turbulent flow. For suffictently high $R e$, theoretical analysis indicates that eddy behavior falls into three distinct size ranges--large eddies which contain most of the turbulent energy of the flow and whose size 1s determined by the phystcal dimensions of the flow, an intermediate range of eddy sizes which are 1sotropic in space and for which the dynamic behavior ts determined solely by the energy transfer rate ( $\varepsilon$ ) from larger to smaller eddies, and the smallest eddies of the flow which are isotropic and energy dissipating. These three types of eddles are often described as being in the energy containing, the 1nertial, or the dissipating ranges. Here the primary interest is the largest and smallest eddies. The sizes of these two types of eddies are characterlzed by length scales which are referred to as the integral (L), and the Kolmogorov ( $n$ ) length scales, respectively.

Values of $L$ are extremely difficult to measure experimentally. Estimates are often obtained by recording the autocorrelation function, $R(T)$, of the 
velocity fluctuations at a point in the flow. The Eulerian integral time scale ( $T$ ) can be obtained from $R(\tau)$. For instance, if $R(\tau$ ) has an exponential dependence on $\tau$, it can be expressed as

$$
R(\tau)=e^{-\tau / T}
$$

Utilizing Taylor's approximation, the value of $L$ can be obtained from $T$ as

$$
L=\bar{U} T
$$

For a coflowing jet with $m=0.33$, Antonia and B1lger [55] measured L values which fell in the $0.73\left(r_{1 / 2}\right)_{u}$ to $0.65\left(r_{1 / 2}\right)_{u}$ range over the downstream distances from $z / r_{0}=80$ to 260. These authors noted that for free jets Wyganski and Feildler [8] reported a value of $L=0.45$ while Corrsin and Ubero1 [56] measured a value of 0.82 . On the basis of these results, it is concluded that values of $L$ for free and coflowing fets are similar and lie in the range from $0.4\left(r_{1 / 2}\right)_{u}$ to $0.8\left(r_{1 / 2}\right)_{u}$.

The Kolmogorov scale can be obtained from dimensional analysis as

$$
n=\left(\frac{\nu^{3}}{\varepsilon}\right)^{1 / 4}
$$

where

$$
\varepsilon=\frac{\left(U^{\prime}\right)^{3}}{L}
$$


Flow fields are generally considered to be fully turbulent when local $\operatorname{Re}$ are sufficlently high to insure the existence of all three eddy size ranges. Bradshaw [51] reports that this condition is satisfled when $L / n>100$.

In general, the flow velocities utilized in this study are not expected to be high enough to result in fully developed turbulent flow behavior. Th1s point has been discussed in detall by Drake et al [58]. In their report, these authors have made detalled comparisons of thelr results for a hydrogen turbulent jet diffusion flame with our earlfer measurements of an isothermal $\mathrm{CH}_{4} /$ alr flow $[1,2]$. As part of this comparison, they estimated values of $\eta=0.01 \mathrm{~cm}$ and $L=0.71 \mathrm{~cm}$ for the length scales of our jet at $z / r_{0}=35$ and $r /\left(r_{1 / 2}\right)_{c}=1.2$. Similar estimates would be expected for the other gas flows Investigated in the present work. Based on the criterion of Bradshaw [51], these jets are not fully turbulent. Drake et al reached the same conclusion based on a similar criterion given by Saffman [57].

It can be shown that one way to meet the criterla of fully developed flow for a given gas pair is to increase the Re of the axisymmetric jets [54]. We have already discussed how values of $C_{m}^{\prime} / \bar{C}_{m}$ are efther constant or slowly varying after an Inftial development region near the jet nozzle. A similar behavior is also expected and has been experimentally observed for the centerline velocity fluctuation intensity, $U_{m}^{\prime} / \bar{U}_{m}$. Using eq. (23), it is easy to see that $U_{m}^{\prime}$ is proportional to $\bar{U}_{m}$ which is in turn proportional to $U_{0}$. These proportionalities along with eqs. (73) and (74) lead to the conclusion that $n$ is proportional to $\left(1 / U_{0}\right)^{3 / 4}$ since values of $L$ and local $v$ are expected to be 1ndependent of Re. Since the value of Re 1 s proportional to Uo (see eq. 67), the local value of $n$ will depend on $(R e)^{-3 / 4}$. Thus increasing Re leads to smaller values of $n$ and increasing values for $L / \eta$. 
There is an apparent contradiction in the experimental findings of this study and the discussion just given concerning fully developed turbulent flow. It has been indicated that the turbulent structure of the jets investigated in this study are not fully developed in the classical sense. Yet these jets have a centerline average concentration falloff which approximates that of a flow having a self-similar behavior and are in quantitative agreement with experimental results for jets having much higher Re. Perhaps more disconcerting, at least at first glance, is the observation that the low $\operatorname{Re}$ jets investigated in this study achieve an asymptotic value of unmixedness at much shorter downstream distances than higher Re flows. Achievement of such an asymptote may be considered as a criterion for a fully developed flow field. Using this criterion, the lower Re flows apparently become fully turbulent at shorter downstream distances than flows at higher Re.

This contradiction between experiment and theory disappears if two simple and reasonable assumptions are made:

1. The time-averaged mixing behavior as well as the values of unmixedness are determined primarily by large scale structures; and

2. A finite time and downstream distance are required for the entire length scale structure of the turbulent flow to come into a pseudoequilibrium. The flow length required increases as the turbulent structure extends to smaller scales.

These two assumptions not only remove the contradiction between theory and experiment, but also lead to predictions for the dependencies of unmixedness 
centerline behavior on $R_{\rho}$ and $R e$ which are in qualitative agreement with experimental observations.

Consider initially the effects of varying $R_{\rho}$ on the values of $\bar{Y}_{m}$ and $Y_{m}^{\prime} / \bar{Y}_{m}$ which are observed. Integral analysis has already been employed to show that measurements of centerline concentration for jets having different $R_{\rho}$ should fall on a common curve when $l / \bar{C}_{m}$ values are plotted as functions of $z / r_{\varepsilon}$. The use of the effective radius concept allows the comparison of jets having different initial momentum values due to molecular weight differences. Sunvala et al [19] and Chen and Rodi [5] have argued that the jet angle will be constant. Since the large scale structure should be nearly independent of $\nu$ and the $\operatorname{Re} 1$ s constant for each jet, it is required that the large scale structure be dependent on $R_{\rho}$. It is expected that in real space, the eddies will be shortened in the axial direction relative to the constant density case for positively buoyant jets while the opposite will be true for jets with $R_{\rho}>1$.

The shadowgraphs of the variable density jets give qualitative support for these conclusions. Increases in the lengths of turbulent structures with Increasing $R_{\rho}$ are apparent when jets having the same Re are compared. The structures of flows having higher jet/coflow densfty ratios are longer axlally than flows having the same $\operatorname{Re}$ but lower density ratios simply because the range of $z / r_{\varepsilon}$ viewed is much shorter.

It is impossible to reach conclusions concerning the spreading rate behavior of the variable density flows from the shadowgraphs. The jets having lower values of $R_{\rho}$ appear to spread faster. This observation may be an 111 - 
sion due to shifts in the virtual origins for the spreading angle. A determination of the actual spreading rate behavior must await quantitative measurements of concentration in the radial directions of the various flows.

The conclusion that the axial turbulent structure for constant $\operatorname{Re}$ jets is determined by a length scale of $r_{\varepsilon}$ along with assumption 2 , above concerning the finite length required for growth of the unmixedness to an asymptotic value requires that the growth curves for centerline unmixedness for variable density cases fall on the same curve when plotted in terms of $z / r_{\varepsilon^{*}}$ Figure 29 shows that this prediction is closely matched by experimental measurements covering a wide range of $R_{\rho}$ values. The assumption of a common turbulence structure also predicts a common value of $\left(\mathrm{Y}_{\mathrm{m}}^{\prime} / \overline{\mathrm{Y}}_{\mathrm{m}}\right)$ asym for jets having varying $R_{\rho} \cdot$ This conclusion also agrees with experimental observation.

The variation in the downstream distance required for a turbulent jet to attain $\left(Y_{m}^{\prime} / \bar{Y}_{m}\right)$ asym with Re also follows naturally from assumptions 1. and 2 . The large scale structure of the flow is expected to be nearly invariant with $\operatorname{Re}$ as long as the $\operatorname{Re}$ is high enough to insure the development of the large scale turbulent eddies. It has already been shown that as the $\operatorname{Re}$ is increased, values of $n$ are expected to decrease as $\operatorname{Re}^{3 / 4}$ with the result that the turbulent eddies extend to smaller sizes. The effect can be clearly seen In the shadowgraphs of the $\mathrm{C}_{3} \mathrm{H}_{8}$ /air and $\mathrm{SF}_{6}$ /air flows at different Re where the apparent observed turbulent structure becomes finer as the $\operatorname{Re}$ is increased. These observations are almost certainly due to the reduction of $n$ at higher Re. 
The centerline concentration falloff has been shown to have a hyperbolic dependence on $z / r_{0}$ which, except for shifts in the virtual origin, is nearly independent of $\mathrm{Re}$ for a given jet/coflow gas pair. This observation is consistent with the assumption that the mixing behavior is determined primarily by the large scale structure of the flow field which is independent of Re. Assumption 2. leads to the prediction that the rate of growth to and the downstream distance required for the attainment of $\left(\mathrm{Y}_{\mathrm{m}}^{\prime} / \overline{\mathrm{Y}}_{\mathrm{m}}\right)$ asym increase with Re. Figures 36-38 show that experimental measurements for $\mathrm{C}_{3} \mathrm{H}_{8} / \mathrm{aIr}, \mathrm{CF}_{4} / \mathrm{air}$, and $\mathrm{SF}_{6} /$ alr flows all conform to these predictions. The observed downstream shift in the growth of unmixedness is also sufficient to explain the observed downstream shift in virtual origins for a given flow with increasing values of $\mathrm{Re}$.

The use of assumptions 1 . and 2 . have allowed the generation of a number of qualitative predictions concerning the centerline concentration behavior of axisymmetric jets which are in agreement with experimental observations. Perhaps the most important conclusion of this analysis is that the criterta normally employed for determining whether or not a turbulent flow is fully developed are too stringent for the role in which they are sometimes used, namely, that of determining whether or not comparison of jet measurements at different Re are valld. Clearly, the nature of the measurement must be considered.

\subsection{Validity of Generalizing Results of This Study to Momentum-Drłven Free Jets}

Much of the discussion above has dealt with the comparison of the results from this study with simflar measurements in free jet flows as well as a 
theoretical development which assumes the lack of wall, coflow, or buoyancyInduced momentum effects on the turbulent mixing. The 1mplicit assumption which has been made throughout the discussion is that the flows which have been Investigated are close approximations to those which would be found for free jets. Since examples have already been discussed where wall effects are present (He/aIr) and buoyancy strongly modifles the flow behavior (the fountains observed in the $\mathrm{SF}_{6} / \mathrm{alr}$ and $\mathrm{SF}_{6} / \mathrm{He}$ flows), it is important to question the validity of this assumption. It w111 be shown that for most of the flows studied the concentration behavior should indeed be that expected for the corresponding free jets. In section 2.3 possible modifications due to buoyancy and the utilization of a coflow within an enclosure have been discussed. This discussion forms the foundation for the remarks which follow.

The confined He/air flow apparently interacts with walls of the enclosure and/or is effected by recirculation eddies in the surrounding coflow. This conclusion is based primarily on the observation that the unmixedness values for this flow falloff as a function of downstream distance while the comparable measurements for the free jet have an unmixedness behavior which is similar to that found for the other jet/coflow gas pairs investigated. Flow visualization results show that the other jets have grown to much smaller radial diameters at the maximum downstream distance for which measurements have been made. Furthermore, as can be seen from table 2 , values of $z_{d} / I_{0}$ and Ct for these other flows are such that recirculation effects should be absent. It is therefore concluded that direct wall interaction of the jet or recirculation in the coflow is important only for the He/air jet at the downstream distances observed in this study. 
No pressure gradient measurements have been made in this study. Strong pressure gradients can modify the turbulent mixing behavior of axisymmetric jets within enclosures [12]. Based solely on comparisons of the findings of this study with those for free jets, such effects have been assumed to be $\operatorname{sma11.}$

Surrounding a jet with a coflow instead of stagnant surroundings modifies the turbulent behavior of the jet (see the discussion in section 2.3). Values of $\mathrm{Ct}$ for the varlous flows studied here are listed in table 2 . Most of the values fall in the 0.8 to 0.9 range. These $C t$ values have been calculated assuming a cylindrical enclosure of the same cross sectional area as the square enclosure actually employed. Based on the constant density measurements of Becker et al [26], a value of $C t=0.67$ corresponds to a jet 1nto a confined coflow which is indistinguishable from a free jet into stagnant surroundings. The variable density Ct derived by Steward and Guruz [29] has been shown to provide an equivalent description for variable density flows. Most of the Ct for the flows investigated in the current study are only slightly larger than 0.67. The findings of Becker et al [26] 1ndicate that very minor variations in centerline mixing behavior will be found for these flows compared to those with $C t=0.67$. On this basis, we conclude that, w1th the exception of the He/air flow and the low Re flows of $S F_{6} / a 1 r$ and $S F_{6} / \mathrm{He}$ discussed below, the jets investigated in this study will have a centerline concentration behavior which is very simflar to the corresponding free jets.

In section 2.3 an approximate analysis assuming stagnant surroundings was used to obtain an expression for the downstream location $\left(z_{\max }\right.$ ) of the top of the fountain formed by a negatively buoyant jet. The presence of a coflow 
would be expected to substantially modify the formation of such a fountain. Discussions in Turner [24] and Chen and Rodi [5], as well as common experience, indicates that such fountains are formed when jet fluid on the centerline comes to a standstill and then falls through the less dense fluid surrounding the jet. If the surrounding gas is flowing, the resulting momentum counteracts the negative buoyancy of the jet fluid and the value of $z_{\max }$ is expected to increase. We are not aware of any theoretical analysis which deals with this complex flow phenomenon. Such an analysis is beyond the scope of this work. It is worthwhile to note that both the approximate analysis given in section 2.3 and the experimental correlation of Turner [24] (eq. (59)) do give rough agreement with experimental results (see table 7 ) despite the fact that both assume stagnant surroundings.

The importance of deviations from purely momentum-driven jets due to buoyancy are difficult to quantify. An approximate expression (eq. 56) was given in section 2.3 which allows the ratio $\left(R_{m}\right)$ of the initial momentum of the flow to that imparted by buoyancy to be calculated as a function of $z / r_{0}$. Values of $R_{m}(z)$ at the most distance $z / r_{0}$ for which a given set of measurements has been made are listed in table 2. From these calculations, it is clear that buoyancy effects should be very small for the $\mathrm{He} / \mathrm{air}, \mathrm{CH}_{4} / \mathrm{air}$, and $\mathrm{C}_{3} \mathrm{H}_{8} / \mathrm{CO}_{2}$ flows. Such a conclusion cannot be definitely made for the other jets which have larger values of $R_{\rho}$. The results given in table 2 indicate that for jets with $R_{\rho}$ greater than 1 the momentum of the flow will have fallen substantially by the time the flow has reached the end of the observation region. If variations in flow momentum are affecting the experimental measurements, the modifications should be most evident for these flows. 
Fortunately, the flows having larger values of $R_{\rho}$ have been studied at variable Re. As expected, increasing Re led to a rapid :eduction in the importance of buoyancy. This can be seen clearly by comparing the values of $R_{m}$ at $z / r_{0}=63$ for the three different $C_{3} H_{8} /$ air jets listed in table 2. For the $\operatorname{Re}=11,880$ case, the value of $R_{m}$ has decreased to 24.0 and this flow should behave very much like a purely momentum-driven fet. Comparison of the centerline measurements of $\bar{Y}_{m}$ and $Y_{m}^{\prime} / \bar{Y}_{m}$ for this gas pair at different flow Re therefore provides a means for assessing the effects of buoyancy on the measurement results.

Figures 32 and 36 show plots of $Y_{0} / \bar{Y}_{m}$ and $Y_{m}^{\prime} / \bar{Y}_{m}$ versus $z / r_{0}$ for the three different $\mathrm{C}_{3} \mathrm{H}_{8} /$ alr flows. The results of the measurements are summarized in tables 5 and 10. Comparison of values of $K_{c}$ for the three fets shows that the slopes of these plots are identical within experimental accuracy. However, downstream shifts are found for the virtual origins ( $\left.z_{0}\right)_{y}$ as the $\operatorname{Re}$ is increased. It has been shown that simflar shifts in virtual orfgin have been found for constant density turbulent flows. Thus, while it is possible that there is a buoyancy effect on virtual origin positfon, it is believed that the primary source of these variations is a Re dependence. Using simflar reasoning, the behavior of the centerline unmixedness is also found to be unaffected by buoyancy effects. Both the asymptotic value and unmixedness development along the jet centerline for the $R e=3960$ flow are consistent with jets having lower values of $R_{\rho}$ in which buoyancy effects should be absent. Values of $S$ and $K$ are also found to be invariant with $\operatorname{Re}$ and to have Jet centerline variations which are determined primarily by the jet to coflow density rat 10. It must be concluded that there are no Indications of buoyancy effects in the experimental measurements for these three $\mathrm{C}_{3} \mathrm{H}_{8}$ /atr flows. 
The absence of a measurable buoyancy effect on the centerline concentration behavior of variable $\mathrm{Re} \mathrm{C}_{3} \mathrm{H}_{8}$ /air flows requires further explanation. The values of $R_{m}(z)$ listed in table 2 for the three flows suggest that the total momentum of the flow at the lower $\operatorname{Re}$ is reduced considerably from Its initial level at $z=0$ due to a substantial buoyancy-induced component. Equation (55) indicates that the negative momentum contribution due to buoyancy has a squared dependence on $z$. This requires that the importance of buoyancy-induced momentum changes decreases rapidly upstream. Apparently, the measurement results for the $\mathrm{C}_{3} \mathrm{H}_{8}$ /air flows at a given downstream distance are determined primarily by the flow conditions upstream, (i.e., during passage through regions where buoyancy contributions to the flow momentum are small). Presumably, measurements made at locations still further downstream would be strongly modified by buoyancy.

Some indication of the modifications in turbulent behavior which are to be expected for these negatively buoyant flows can be found in the results for the $\mathrm{SF}_{6}$ turbulent jets at $\operatorname{Re}=3950$ flowing into coflows of He and air. Figure 21 shows the results of experimental measurements of average centerline concentration for the two flows plotted as $Y_{0} / \bar{Y}_{m}$ versus $z / r_{0}$. Both plots show an initial region at upstream positions for which the data have a roughly linear dependence on $z / r_{0}$. This is the same dependence which has been observed for other jet/coflow gas pairs in which buoyancy effects are absent. However, as $z / r_{0}$ is increased, the jet begins to slow down significantly due to buoyancy and values of $\mathrm{Y}_{\mathrm{o}} / \overrightarrow{\mathrm{Y}}_{\mathrm{m}}$ increase dramatically until the downstream position where the tops of the fountains form. These findings suggest that as the momentum of the flow decreases significantly, the centerline mixing rate increases. 
The results of calculations of $R_{m}$ at $z / r_{0}=63$ for the $C F_{4} /$ air flow at $\operatorname{Re}=3960$ and the $\mathrm{SF}_{6} /$ air flow at $\mathrm{Re}=7890$ are included in table 2 . For these two jets the effects of buoyancy on the flow momentim are quite large at this downstream position. However, comparisons of experimental results for these flows with the corresponding flows at higher Re show nc indication of variations in centerline concentration behavior due to buoyancy. As is the case for the $\mathrm{C}_{3} \mathrm{H}_{8}$ /air flows, the integrated effects of buoyancy are too small to be detected as variations in the average concentration or unmixedness values over the range of downstream distances investigated. The experimental results for these flows must correspond closely to those which would be found for purely momentum driven flows.

The above discussion indicates that most of the flows which have been investigated during this study will be good approximations of the corresponding free jet flows and that buoyancy effects on the measurements at the relatively short downstream distances investigated are minimal.

\section{FINAL REMARKS}

This work has reported flow visualization studies and centerline concentration measurements for axisymmetric jets of gases into slow coflows of a second gas. In this manner it has been possible to fnvestigate turbulent flows where values of the density ratios of the jet to surrounding gases vary from 0.14 to 37 . It is interesting to note that if this range of values of density ratio were due to gas temperature variations of a single ideal gas, the corresponding temperature range would extend from 8 to $2100 \mathrm{~K}$. To our knowledge, this is the most extensive range of global gas denstey variations ever systematically investigated within a single experimental flow system. 
There are a number of experimental parameters which may influence the flow behavior. Potential effects due to the experimental requirement for the use of an enclosure and coflowing gas as well as flow momentum variations due to buoyancy have been discussed. In many cases, approximate treatments have been utflized in order to estimate the variations in experimental results expected from those which would be found for purely momentum-driven, turbulent free jets. In general, these treatments and comparisons with experimental results have led to the conclusion that such effects are small. However, it cannot be claimed that this study has fully characterized such effects. Their possible influence on the experimental findings as well as the validity of the conclusions which have been drawn from the results must be evaluated in terms of this incomplete characterization.

The findings of this study have been compared whenever possible to other literature measurements. Unfortunately, despite the intense experimental and theoretical interest in axisymmetric jets and the avallability of a large number of studies, it is extremely difficult to reach defintte conclusions concerning the effects of such important flow parameters as initial flow conditions, Re, and jet to surrounding gas density ratio on the turbulent mixing behavior.

The lack of a concensus on the existence of an asymptotic centerline unmixedness is an example of the difficulty. For instance, in a very recent study, Dahm [16] has concluded that such an asymptote does not exist. This led this author to suggest a new type of concentration similarity parameter for treating the concentration fluctuation behavior of these jets. On the other hand, the findings of the current study do indicate the existence of an 
asymptotic value for centerline unmixedness and a very simple, qualitative theory based on variations in unmixedness development with $R e$ and $R_{\rho}$ has been suggested as an explanation for the variations of results among different studies. The answer to the question of whether or not such an asymptote exists is very important to both experimentalists and theoreticians who are trying to characterize turbulent flows of this type. Clearly, the question has not been answered satisfactorily and more experimental and theoretical study is necessary to solve this important puzzle.

Similarity analysis has been employed in this study as a means for comparing jets having different global density ratios and Re. For example, the fitting of the centerline concentration data plotted as $Y_{0} / \bar{Y}_{\text {m }}$ versus $z / r_{0}$ to a straight line is based on the assumption of self-similarity. Selfsimilarity is also the basis of the effective radius concept as originally developed by Thring and Newby [18]. These conclusions have been utilized freely despite the fact, as discussed in sections 2.2 and 2.3 , that true selfsimizarity is not possible for elther jets into a coflow or for jets into surroundings at a different density. Future work will be necessary to determine whether such observations as the incomplete correlation of the average centerline concentration data in terms of eq. (24) for varlable density flows has its basis in a breakdown of self-similarlty or in some other effect.

The use of both shadowgraphy for flow visualization and Raylelgh 11 ght scattering concentration measurements has allowed much to be learned concerning centerline mixing in these turbulent flows. It is clear, however, that a more complete understanding can be achieved by extending these measurements to radial locations in the jets and by obtaining velocicy luiormation 
for the flow field. A method for making slmultaneous concentration and veloc1ty measurements has been previously described [59]. In the near future, we plan to utilize this technique to map out the axial and radial concentration and velocity fields for the varlable density jet/coflow gas pairs 1nvestigated In this study.

As for most Investigations of this type, the findings of this study have proven to be somewhat inconclusive and have perhaps ralsed more questions than have been answered. However, the detalled nature of the quantitative measurements is such that very clear dependencles of flow behavior on $R_{\rho}$ and $R e$ have been elucidated. Many of these findings have not been reported or are not widely quoted in the 11terature. For this reason, and at the same time keepling in mind the caveats noted above, this report concludes by 118 ting the most Important findings concerning density and Re effects on mixing for an axisymmetric turbulent gas jet flowing into a second gas.

(1) General conclusions drawn from Integrated flow visualization studies agree with those based on the quantitative centerline concentration results.

(2) The average centerline concentration behavior for fets and coflows having different density ratios all have linear dependencies when values recorded for $z / r_{0}>20$ are plotted as $Y_{0} / \bar{Y}_{m}$ versus $z / r_{0}$.

(3) The use of the effective radius concept of Thring and Newby [18] gives a partial correlation of the concentration results for vartable density flows. An improved correlation is found if the square 
root dependence on density ratio in eq. (22) is changed to $\left(\rho_{0} / \rho_{\infty}\right)^{0.6}$

(4) Virtual origins for the concentration plots are highly dependent on density ratio, going from negative to positive values as $R_{\rho}$ is decreased from values greater than to values less than 1 . This behavior is predicted qualitatively by eq. (34) which is based on an approximate solution of the integral equations.

(5) The centerline unmixedness values in terms of $Y_{m}^{\prime} / \vec{Y}_{m}$ have a common asymptote of 0.23 for all of the flows which have been Investigated.

(6) The effective radius concept provides a good collapse of the centerline unmixedness growth curves for flows at the same Re, but having different values of $R_{\rho}$.

(7) Values of $S$ and $K$ for the centerline concentration fluctuations are close to the1r Gaussian values of $S=0$ and $K=3$, but vartations with $R_{\rho}$ are found.

(8) Increasing Re shift the pos1tions of the virtual or1gins downstream for plots of $Y_{0} / \bar{Y}_{m}$ versus $z / r_{0}$, but has no measurable effect on the slope.

(9) The asymptotic value of centerline unmixedness is Independent of Re, but the downstream distance required to atta1n $\left(Y^{\prime} / \bar{Y}_{m}\right)$ asym increases rapldly with $\operatorname{Re}$. 
(10) Values of $S$ and $K$ as functions of $z / r_{0}$ are insensitive to $R e$.

\section{ACKNOWLEDGMENTS}

This research has been partially sponsored by the Air Force Office of Scientific Research, Air Force Systems Command, USAF, under Grant Number AFOSR-ISSA-85-00012. The U.S. Government is authorized to reproduce and distribute reprints for Governmental purposes not withstanding any copyright notation thereon.

The author would like to thank Drs. Bernie McCaffrey and Howard Baum of the Center for Fire Research for thorough reviews of this manuscript and their many helpful comments. Thanks are also due to Professor Wolfgang Rodi of the UnIversität Rarlsruhe for providing a description of unpublished research and to Dr. Robert Schefer of CFR, Sandia National Laboratories for providing a preprint of work prior to publication.

\section{REFERENCES}

[1] Pitts, W.M. and Kashiwagi, T., The applitcation of laser-induced Rayleigh light scattering to the study of turbulent mixing, Nat. Bur. Stand. (U.S.), NBSIR 83-2641, January 1983, 109 p.

[2] Pitts, W.M. and Kashiwagi, T., The application of laser-induced Rayleigh light scattering to the study of turbulent mixing, J. Fluid Mech. 14i: 391-429, Apri1 1984 .

[3] Graham, S.C., Grant, A.J., and Jones, J.M., Transient molecular concentration measurements in turbulent flows using Rayleigh light scattering, AIAA J. 12(8):1140-1142, August 1974 .

[4] Dyer, T.M., Rayleigh scattering measurements of tine-resolved concentration in a turbulent propane jet, AIAA J. 17(8):912-914, August 1979. 
[5] Chen, C.J. and Rodi, W., Vertical turbulent buoyant jets--a review of experimental data, Pergamon Press, New York, 1980, 83 p.

[6] Corrsin, S. and Ubero1, M.S., Further experiments on the flow and heat transfer in a heated turbulent air jet, NACA TN 1865, Apri1 1949.

[7] List, E.J., Mechanics of turbulent buoyant jets and plumes, in Turbulent Buoyant Jets and Plumes, W. Rodi, ed., Pergamon Press, New York, 1982, $1-68$.

[8] Wygnansk1, I. and Fledler, H., Some measurements in the self-preserving jet, J. Fluld Mech. 38(3):577-612, September 1969.

[9] Becker, H.A., Hottel, H.C., and Willlams, G.C., The nozzle-fluid concentration fleld of the round, turbulent, free jet, J. Fluid Mech. $30(2): 285-303$, November 1967 .

[10] Birch, A.D., Brown, D.R., Dodson, M.G., and Thomas, J.R., The turbulent concentration field of a methane jet, J. Fluld Mech. 88(3):431-449, October 1978.

[11] Bradshaw, P., The effect of Inftial conditions on the development of a free shear layer, J. Fluid Mech. 26(2):225-236, October 1966.

[12] Harsha, P.T., Free turbulent mixing: a critical evaluation of theory and experiment, Arnold Eng. Dev. Cen. Report AEDC-TR-71-36, February $1971,630 \mathrm{p}$.

[13] Alexander, L.G., Baron, T., and Comings, E.W., Transport of momentum, mass, and heat in turbulent jets, Univer. of Illinols Exp. Stat. Bulletin 413, May 1953.

[14] Ebrahiml, I., Axlaler Verlauf der Geschwind1gke1t in Luft-Freistrahlen, Forsch. Ing.-Wes. 42(1):33-35, 1976.

[15] Ebrahim1, I. and Kle1ne, R., Konzentrationsfelder in isothermen LuftErelstrahlen. Forsch. Ing. -Wes. 43(1):25-30, 1977.

[16] Dahm, W.J.A., Experiments on entrainment, mixing, and chemical reactions in turbulent jets at large Schmidt number, Dissertation, California Institute of Technology, May 1985, $161 \mathrm{p}$.

[17] O'Connor, T.J., Comfort, E.H., and Cass, L.A., Turbulent mixing of an axisymmetric jet of partially dissoclated nitrogen with amblent a1r, AIAA J. 4(11):2026-2032, November 1966.

[18] Thring, M.W. and Newby, M.P., Combustion length of enclosed turbulent jet flames, Fourth Symposium (International) on Combustion, P1ttsburgh, The Standing Committee on Combustion, 1953, 789-796.

[19] Sunavala, P.D., Hulse, C., and Thring, M.W., Mixing and combustion in free and enclosed turbulent jet diffusion flames, Com. Flame 1(2): 179-193, June 1957. 
[20] Wilson, R.A.M. and Danckwerts, P.V., Studies in turbulent mixing--II a hot jet, Chem. Eng. Science 19:885-895, 1964 .

[21] Ricou, F.P. and Spalding, D.B., Measurements of entrainment by axisymmetrical jets, J. Fluid Mech. 11:21-32, 1961 .

[22] Hill, B.J., Measurement of local entrainment rate in the initial region of axisymmetric turbulent air jets, J. Fluid Mech. 51(4):773-779, February 1972 .

[23] Rodi, W., private communication.

[24] Turner, J.S., Jets and plumes with negative or reversing buoyancy, J. Fluid Mech. 26(4):779-792, December 1966.

[25] Abramovich, G.N., The theory of turbulent jets, The MIT Press, Cambridge, MA, 1963, 670 p.

[26] Becker, H.A., Hottel, H.C., and Williams, G.C., Mixing and flow in ducted turbulent jets, Ninth Symposium (International) on Combustion, Pittsburgh, PA, The Combustion Institute, 1962, 7-20.

[27] Craya, A. and Curtet, R., Sur l'evolution d'un jet in espace confine, Compt. Rend 241(8):621-622, August 1955 .

[28] Curtet, R., Confined jets and recirculation phenomena with cold air, Com. Flame 11(4):383-411, December 1958 .

[29] Steward, F.R. and Guruz, A.G., Aerodynamics of a confined jet with variable density, Com. Science Tech. 16(1-2):29-45, July 1977 .

[30] Pitts, W.M. and McCaffrey, B.J., Response behavior of hot-wires and films to flows of different gases, Nat. Bur. Stand. (U.S.), NBSIR 85-3203, July 1985, 123 p.

[31] Goldstein, R.J., Optical systems for flow measurement: shadowgraph, schlieren, and interferometric techniques, Chapter 8 in Fluid Mechanics Measurements, Richard J. Goldstein, ed., Hemisphere Publishing, Washington, DC, 1983, 377-422.

[32] Weinberg, F.J., Optics of flames, Butterworths, Washington, DC, 1963, 251 p.

[33] Pike, E.R., Photon statistics, Riv. Nuovo Cimento 1 (Numero Speciale): 277-314, 1969 .

[34] Arcoumanis, C., A laser Rayleigh scattering system for scalar transport studies, Exp. Fluids 3(2):103-108, 1985.

[35] Hinze, J.0. and van der Hegge Zijnen, B.J., Transfer of heat and matter in the turbulent mixing zone of an axially symetrical jet, Appl. Sci. Res. Al (5-6):435-461, 1947. 
[36] Keagy, W.R. and Weller, A.E., A study of freely expanding inhomogeneous jets, Proceedings 1949 Heat Transfer and Fluid Mechanics Institute, Berkeley, CA, May 1949, 89-98.

[37] McQuaid, J. and Wright, W., Turbulence measurements with hot-wire anemometry in non-homogeneous jets, Int. J. Heat Mass Trans. $17(2): 341-349$, February 1974 .

[38] Chassaing, P. and Claria, A., Transfer de masse dans des jets turbulents de revolution en milieu non homogene, Int. J. Heat Mass Trans. 19(3):249-258, March 1976.

[39] Lockwood, F.C. and Moneib, H.A., Fluctuating temperature measurements in a heated round free jet, Com. Science Tech. 22(1-2):63-81, Apri1 1980 .

[40] Birch, A.D., Brown, D.R., Dodson, M.G., and Swaffield, F., The structure and concentration decay of high pressure jets of natural gas, Com. Science Tech. 36(5-6):249-261, April 1984 .

[41] Niwa, C., Ichizawa, J., Yoshikawa, N., and Ohtake, K., Time-resolved concentration measurements of jets by laser Rayleigh method, Proceedings of the Fourteenth International Symposium on Space Technology and Sclence, Tokyo, 1984 .

[42] Schefer, R.W. and Dibble, R.W., Rayleigh scattering measurements of mixture fraction in a turbulent nonpremixed propane jet, to be published.

[43] Keagy, W.R. and Ellis, H.H., The application of the schlieren method to the quantitative measurement of mixing gases in jets, Third symposium on Combustion, Flame, and Explosion Phenomena, Williams and Wilkins, Baltimore, MD, 1949, 667-674.

[44] Uberoi, M.S. and Garby, L.C., Effect of density gradients on an air jet, Phys. Fluids Supp. 10:S200-S202, 1967.

[45] Shaughnessy, E.J. and Morton, J.B., Laser light-scatterłng measurements of particle concentration in a turbulent jet, J. Fluid Mech. $80(1)$ : 129-148, April 1977.

[46] Rosenweig, R.E., Hottel, H.C., and W1lliams, G.C., Smoke-scattered l1ght measurement of turbulent concentration fluctuations, Chem. Eng. Science $15: 111-129,1961$.

[47] Way, J. and Libby, P.A., Application of hot-wire anemometry and digital techniques to measurements in a turbulent helfum jet, AIAA J. 9(8): 1567-1573, August 1971 .

[48] Nakamura, I., Saka1, Y., and Miyata, M., A study of the fluctuatıon concentration field in a turbulent jet, Mem. Fac. Eng., Nagoya Univer. 34(1):113-124, 1982 . 
[49] Ebrahimi, I. and Kleine, R., The nozzle fluid concentration fluctuation field in round turbulent free jets and jet diffusion flames, Sixteenth Symposium (International) on Combustion, The Combustion Institute, Pittsburgh, PA, 1977, 1711-1723.

[50] Ebrahimi, I., Günther, R., and Haberda, F., Wahrscheinlichkeitsdichteverteilungen der Konzentration in isothermen Luft-Freistrahlen, Forsch. Ing.-Wes. 43(2):47-52, 1977.

[51] Bradshaw, P., An introduction to turbulence and its measurement, Pergamon Press, New York, 1971,218 p.

[52] Hinze, J.0., Turbulence, 2nd ed., McGraw-Hill, New York, 1975, 790 p.

[53] Tennekes, H. and Lumley, J.L., A first course in turbulence, The MIT Press, Cambridge, MA, 1972, 300 p.

[54] Landau, L.D. and Lifshitz, E.M., Fluid Mechanics, Pergamon Press, London, $1959,536 \mathrm{p}$.

[55] Antonia, R.A. and Bilger, R.W., An experimental investigation of an axisymmetric jet in a co-flowing air stream, J. Fluid Mech. 61(4): 805-822, December 1973.

[56] Corrisin, S. and Uberoi, M., Spectra and diffusion in a round turbulent jet, N.A.C.A. Report 1040:915-935, 1951.

[57] Saffman, P.G., Problems and progress in the theory of turbulence, in Structure and Mechanisms of Turbulence II, Vol. 2, H. Fieldler, ed., Lecture notes in physics, Vol. 76, Springer-Verlag, New York, 1978, 273-306.

[58] Drake, M.C., Shyy, W., and Pitz, R.W., Superlayer contributions to conserved scalar pdfs in a $\mathrm{H}_{2}$ turbulent jet diffusion flame, Proceedings of the Fifth Symposium on Turbulent Shear Flows, August 7-9, 1985, Ithaca, NY.

[59] Pitts, W.M., McCaffrey, B.J., and Kashiwagi, T., A new diagnostic technique for simultaneous, time-resolved measurements of concentration and velocity in simple turbulent flow systems, Proceedings of the Fourth Symposium on Turbulent Shear Flows, September 12-14, 1983, Karlsruhe, W. Germany. 


\section{NOMENCLATURE}

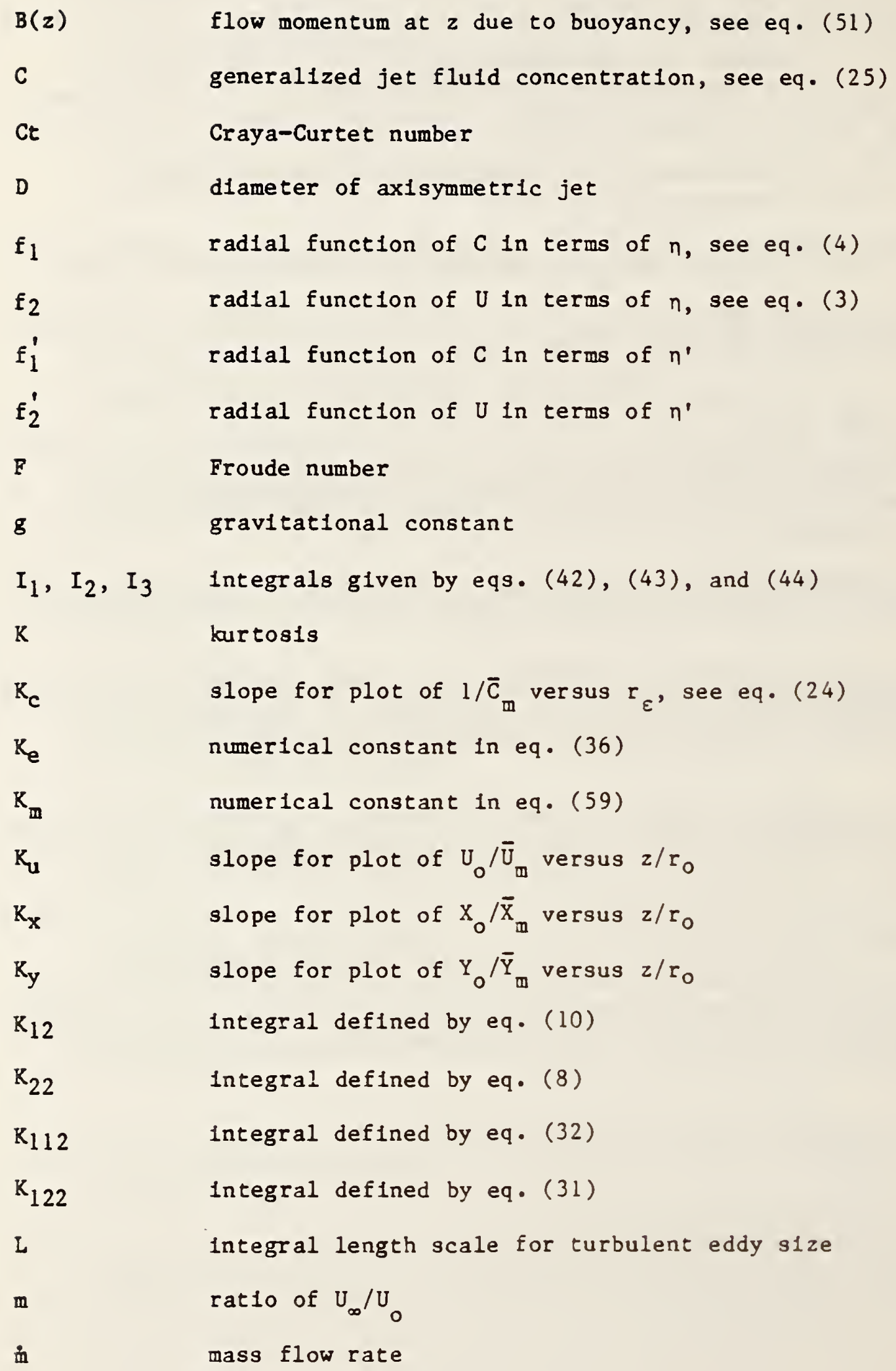


momentum flux

jet fluid concentration flux

radial coordinate

radius of cylindrical enclosure

effective radius, see eq. (22)

radial distance for which measured value of scalar or velocity is equal to one half of the jet centerline value

autocorrelation of velocity fluctuations

$\left(r_{1 / 2}\right)_{c} /\left(r_{1 / 2}\right)_{u}$

ratio of flow momentum due to initial momentum of jet to that due to buoyancy

ratio of initial jet gas density to the surrounding gas density

Reynolds number for jet, see eq. (67)

skewness

Schmidt number

Eulerian integral time scale; temperature

flow velocity in axial direction of jet

kinematic velocity, see eq. (61)

dynamic velocity, see eq. (62)

mole fraction of jet gas

mass fraction of jet gas

axial coordinate

dummy variable for integration, see eq. (51)

axial location where complete entrainment of a coflow by a jet occurs

axial location of the top of the fountain formed by a negatively buoyant jet

axial location of virtual origin for plots of inverse average concentration or inverse velocity versus downstream distance 


\section{$z_{0}^{\prime}$}

$z_{1 / 2}$

\section{Greek letters}$$
\rho
$$$$
\rho^{\prime}
$$

\section{Subscripts}

\section{as ym}

c

f

m

o

tu

u

$\mathbf{x}$ axial location of virtual origin for the inverse rms of concentration versus downstream distance

axial distance for which a centerline value of a scalar is one half of its value at $z=0$

gas density

term defied by eq. (66)

rate of energy cascade through turbulent eddies

dynamic viscosity

kinematic viscosity

nondimensionalized similarity parameter, r/z

Kolmogorov length scale

nondimensionalized local similarity parameter, $r / r_{1 / 2}$

half angle for jet

value at asymptote

value for generalized concentration

scalar value in downstream region of jet where it can be treated as a constant

value of velocity or scalar along the jet centerline

value at the jet exit, or with $z$, a virtual origin

value for a turbulent flow

value for axial velocity

value in terms of mole fraction of jet gas

value in terms of mass fraction of jet gas

value in gas surrounding jet

gas 1

gas 2 
Superscripts

overbar time-averaged value

rms of fluctuating value 
Table 1. Suppliers and Purities of Gases Used in Th1s Work

$\underline{\text { Gas }}$

$\mathrm{He}$

$\mathrm{CH}_{4}$

air

$\mathrm{CO}_{2}$

$\mathrm{C}_{3} \mathrm{H}_{8}$

$\mathrm{CF}_{4}$

$\mathrm{SF}_{6}$
Supplier

Air Products

Matheson

house line

Roberts Oxygen

Matheson

Dupont

Mathes on
Purity

$>99.995 \%$

$>98 \%$

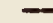

$>99.9 \%$

$>99 \%$

$>99.7 \%$

$>98.8 \%$ 


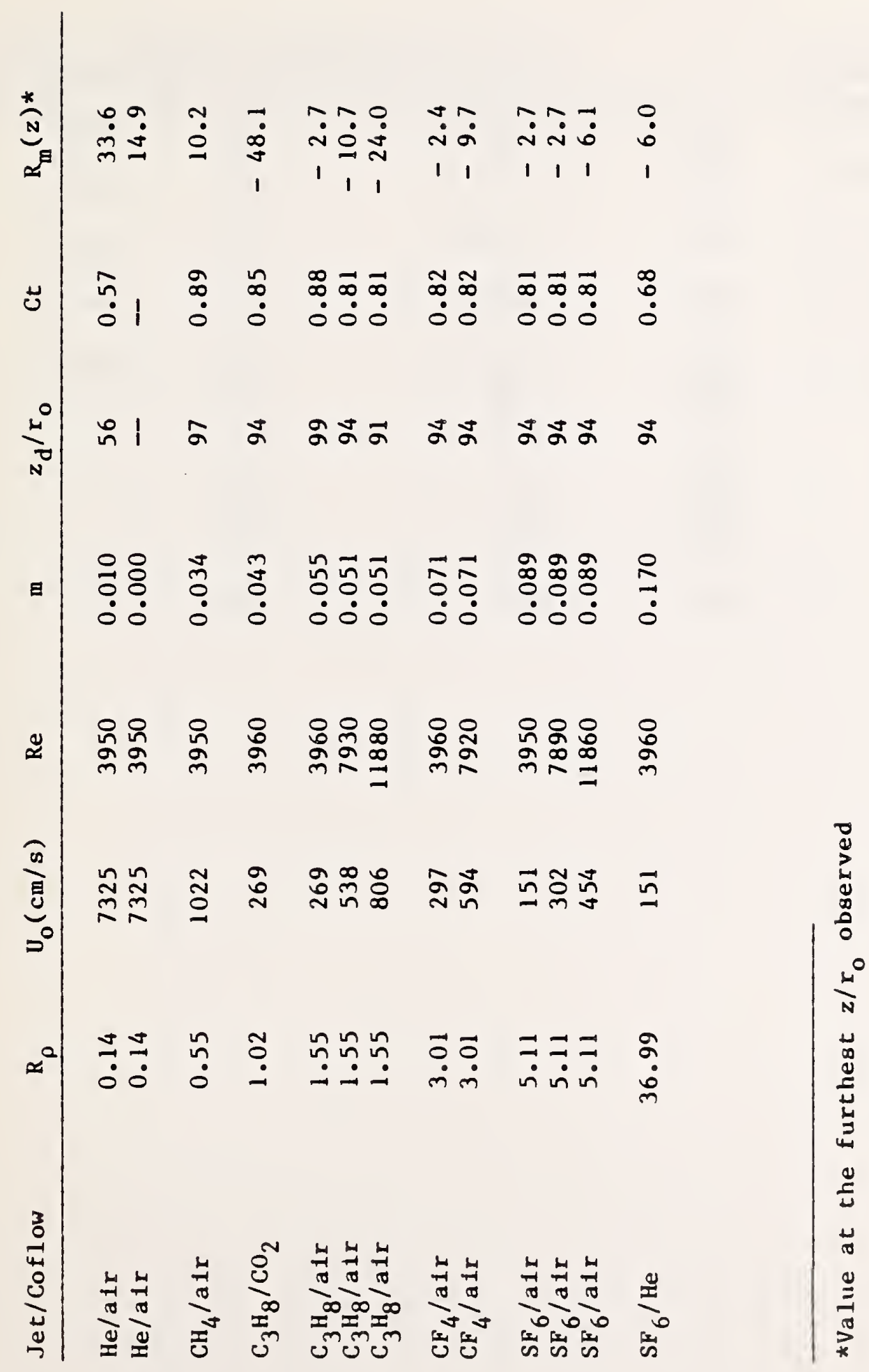


Table 3. Estimated Values from Shadowgraphs

\begin{tabular}{|c|c|c|c|c|}
\hline Jet/Coflow & $\operatorname{Re}$ & $\mathrm{R}_{\rho}$ & $\begin{array}{l}z / r_{0} \text { for Initial } \\
\text { Vortex Roll Up }\end{array}$ & $\begin{array}{c}D / r_{0} \text { at } \\
z / r_{0}=40\end{array}$ \\
\hline $\begin{array}{l}\text { He/air } \\
\text { free He/air }\end{array}$ & $\begin{array}{l}3950 \\
3950\end{array}$ & $\begin{array}{l}0.14 \\
0.14\end{array}$ & $\begin{array}{l}0.8 \\
1.4\end{array}$ & $\begin{array}{l}20 \\
20\end{array}$ \\
\hline $\mathrm{CH}_{4} / \mathrm{air}$ & 3950 & 0.55 & 1.6 & 16 \\
\hline $\mathrm{C}_{3} \mathrm{H}_{8} / \mathrm{CO}_{2}$ & 3960 & 1.02 & 1.9 & 14 \\
\hline $\begin{array}{l}\mathrm{C}_{3} \mathrm{H}_{8} / \text { air } \\
\mathrm{C}_{3} \mathrm{H}_{8} / \text { air } \\
\mathrm{C}_{3} \mathrm{H}_{8} / \text { air }\end{array}$ & $\begin{array}{l}3960 \\
3960 \\
3960\end{array}$ & $\begin{array}{l}1.55 \\
1.55 \\
1.55\end{array}$ & $\begin{array}{l}2.5 \\
1.3 \\
1.1\end{array}$ & $\begin{array}{l}14 \\
14 \\
14\end{array}$ \\
\hline $\begin{array}{l}\mathrm{SF}_{6} / \text { air } \\
\mathrm{SF}_{6} / \text { air } \\
\mathrm{SF}_{6} / \text { air }\end{array}$ & $\begin{array}{r}3950 \\
7890 \\
11860\end{array}$ & $\begin{array}{l}5.11 \\
5.11 \\
5.11\end{array}$ & $\begin{array}{l}3.4 \\
2.8 \\
2.2\end{array}$ & $\begin{array}{l}- \\
10 \\
10\end{array}$ \\
\hline $\mathrm{SF}_{6} / \mathrm{He}$ & 3950 & 37.0 & 5.6 & -- \\
\hline
\end{tabular}


Table 4. Predicted and Observed Rayleigh Scattering Cross Section Ratios

Gas Pair

$\mathrm{CH}_{4}$ : air

$\mathrm{C}_{3} \mathrm{H}_{8}$ :air

$\mathrm{CO}_{2}$ : air

$\mathrm{CF}_{4}$ :air

$\mathrm{SF}_{6}: \mathrm{air}$ $\underline{\text { Predicted Ratio }}$

2.34

14.0

2.40

2.68

6.74
Observed Ratio

$2.29 \pm 0.02$

$14.6 \pm 0.2$

$2.48 \pm 0.03$

$2.66 \pm 0.04$

$6.88 \pm 0.05$ 
Table 5. Results for Average Concentration Measurements

\begin{tabular}{|c|c|c|c|c|c|c|}
\hline Jet/Coflow & $\operatorname{Re}$ & $\mathrm{K}_{\mathrm{x}}$ & $\left(z_{0}\right)_{x} / r_{0}$ & $\mathrm{Ky}$ & $\left(z_{o}\right)_{y} / r_{o}$ & $\mathrm{~K}_{\mathrm{c}}$ \\
\hline $\begin{array}{l}\mathrm{He} / \mathrm{alr} \\
\text { free } \mathrm{He} / \mathrm{alr}\end{array}$ & $\begin{array}{l}3950 \\
3950\end{array}$ & $\begin{array}{l}0.038 \\
0.048\end{array}$ & $\begin{array}{r}-17.8 \\
-\quad 9.3\end{array}$ & $\begin{array}{l}0.281 \\
0.344\end{array}$ & $\begin{array}{l}5.5 \\
8.9\end{array}$ & $\begin{array}{l}0.104 \\
0.128\end{array}$ \\
\hline $\mathrm{CH}_{4} / \mathrm{air}$ & 3950 & 0.085 & -3.4 & 0.154 & 1.9 & 0.115 \\
\hline $\mathrm{C}_{3} \mathrm{H}_{8} / \mathrm{CO}_{2}$ & | 3960 & 0.113 & -0.7 & 0.113 & -0.7 & 0.114 \\
\hline $\begin{array}{l}\mathrm{C}_{3} \mathrm{H}_{8} / \mathrm{air} \\
\mathrm{C}_{3} \mathrm{H}_{8} / \mathrm{air} \\
\mathrm{C}_{3} \mathrm{H}_{8} / \mathrm{air}\end{array}$ & $\begin{array}{r}3960 \\
7930 \\
11880\end{array}$ & $\begin{array}{l}0.130 \\
0.131 \\
0.128\end{array}$ & $\begin{array}{l}0.1 \\
4.7 \\
6.7\end{array}$ & $\begin{array}{l}0.084 \\
0.084 \\
0.082\end{array}$ & $\begin{array}{r}-4.1 \\
0.4 \\
2.1\end{array}$ & $\begin{array}{l}0.104 \\
0.105 \\
0.103\end{array}$ \\
\hline $\begin{array}{l}\mathrm{CF}_{4} / \mathrm{aIr} \\
\mathrm{CF}_{4} / \mathrm{aIr}\end{array}$ & $\begin{array}{l}3960 \\
7920\end{array}$ & $\begin{array}{l}0.164 \\
0.164\end{array}$ & $\begin{array}{r}5.5 \\
10.2\end{array}$ & $\begin{array}{l}0.055 \\
0.055\end{array}$ & $\begin{array}{l}-7.3 \\
-2.7\end{array}$ & $\begin{array}{l}0.095 \\
0.095\end{array}$ \\
\hline $\begin{array}{l}\mathrm{SF}_{6} / \mathrm{alr} \\
\mathrm{SF}_{6} / \mathrm{air}\end{array}$ & $\begin{array}{r}7890 \\
11860\end{array}$ & $\begin{array}{l}0.166 \\
0.170\end{array}$ & $\begin{array}{r}9.8 \\
12.0\end{array}$ & $\begin{array}{l}0.032 \\
0.034\end{array}$ & $\begin{array}{l}-17.5 \\
-12.8\end{array}$ & $\begin{array}{l}0.072 \\
0.076\end{array}$ \\
\hline
\end{tabular}


Table 6. $K_{c}$ Values which Result when the Exponent in Equation (22) is Set to 0.6

Jet/Coflow

$\mathrm{He} / \mathrm{alr}$

free $\mathrm{He} / \mathrm{alr}$

$\mathrm{CH}_{4} / \mathrm{aIr}$

$\mathrm{C}_{3} \mathrm{H}_{8} / \mathrm{CO}_{2}$

$\mathrm{C}_{3} \mathrm{H}_{8} / \mathrm{aIr}$

$\mathrm{C}_{3} \mathrm{H}_{8}$ /aIr

$\mathrm{C}_{3} \mathrm{H}_{8} /$ aII

$\mathrm{CF}_{4}$ /air

$\mathrm{CF}_{4}$ /air

$\mathrm{SF}_{6} / \mathrm{air}$

$\mathrm{SF}_{6} / \mathrm{air}$
Re

3950

3950

3950

3960

3960

7930

11880

3960

7920

7890

11860
$\mathrm{K}_{\mathrm{c}}$

0.086

0.105

0.108

0.114

0.109

0.110

0.107

0.106

0.106

0.084

0.089 
Table 7. Values of $z_{\max }$ Calculated Using Equations (58) and (59)

$\begin{array}{lrrrr}\text { Flow } & \text { Re } & \text { Eq. }(58) & & \text { Eq. }(59) \\ \mathrm{C}_{3} \mathrm{H}_{8} / \mathrm{CO}_{2} & 3960 & 426 \mathrm{r}_{\mathrm{o}} & 296 \mathrm{r}_{\mathrm{o}} & \\ \mathrm{C}_{3} \mathrm{H}_{8} / \mathrm{air} & 3960 & 100 \mathrm{r}_{\mathrm{o}} & 63 \mathrm{r}_{\mathrm{o}} & \\ \mathrm{C}_{3} \mathrm{H}_{8} / \mathrm{air} & 7930 & 201 \mathrm{r}_{\mathrm{o}} & 126 \mathrm{r}_{\mathrm{o}} & \\ \mathrm{C}_{3} \mathrm{H}_{8} / \mathrm{air} & 11880 & 301 \mathrm{r}_{\mathrm{o}} & 188 \mathrm{r}_{\mathrm{o}} & \\ \mathrm{CF}_{4} / \mathrm{air} & 3960 & 96 \mathrm{r}_{\mathrm{o}} & 51 \mathrm{r}_{\mathrm{o}} & \\ \mathrm{CF}_{4} / \mathrm{air} & 7920 & 191 \mathrm{r}_{\mathrm{o}} & 101 \mathrm{r}_{\mathrm{o}} & \\ \mathrm{SF}_{6} / \mathrm{air} & 3950 & 51 \mathrm{r}_{\mathrm{o}} & 24 \mathrm{r}_{\mathrm{o}} & 38 \mathrm{r}_{\mathrm{o}} \\ \mathrm{SF}_{6} / \mathrm{air} & 7890 & 101 \mathrm{r}_{\mathrm{o}} & 47 \mathrm{r}_{\mathrm{o}} & \\ \mathrm{SF}_{6} / \mathrm{air} & 11860 & 152 \mathrm{r}_{\mathrm{o}} & 71 \mathrm{r}_{\mathrm{o}} & \\ \mathrm{SF}_{6} / \mathrm{He} & 3950 & 75 \mathrm{r}_{\mathrm{o}} & 21 \mathrm{r}_{\mathrm{o}} & 44 \mathrm{r}_{\mathrm{o}}\end{array}$


Table 8. Summary of Average Centerline Concentration Measurements for Axisymetric Murbulent Jets

\begin{tabular}{|c|c|c|c|c|c|c|c|}
\hline $\begin{array}{l}\text { P1rst Author, } \\
\text { Ref., Date }\end{array}$ & Jet/Coflow & $p_{0} / p_{\infty}$ & $D_{0}(\mathrm{~cm})$ & Re & $k_{c}$ & $\left(z_{0}\right)_{c} / z_{0}$ & Method \\
\hline $\begin{array}{l}\text { Blnze, [35], } \\
\text { 1948* }\end{array}$ & a1r/a1r & 1.00 & 2.54 & 67,000 & 0.114 & 4.0 & tracer TC \\
\hline $\begin{array}{l}\text { Corrsin, [6] } \\
\text { 1949* }\end{array}$ & $\begin{array}{l}\text { a1r/a1r } \\
\text { a1r/a1r }\end{array}$ & $\begin{array}{l}0.95 \\
0.50\end{array}$ & $\begin{array}{l}2.54 \\
2.54\end{array}$ & $\begin{array}{l}>33,000 \\
>33,000\end{array}$ & $\begin{array}{l}0.103 \\
0.093\end{array}$ & $\begin{array}{l}2.8 \\
7.6\end{array}$ & $\begin{array}{l}\text { resistance } \\
\text { the rmometry }\end{array}$ \\
\hline $\begin{array}{l}\text { Keagy, }[36], \\
\text { 1949* }\end{array}$ & $\begin{array}{l}\mathrm{He} / \mathrm{alr} \\
\mathrm{N}_{2} / \mathrm{alr} \\
\mathrm{CO}_{2} / \mathrm{aIr}\end{array}$ & $\begin{array}{l}0.14 \\
0.97 \\
1.53\end{array}$ & $\begin{array}{l}0.33 \\
0.33 \\
0.33\end{array}$ & $\begin{array}{r}3,360 \\
26,300 \\
49,800\end{array}$ & $\begin{array}{l}0.107 \\
0.092 \\
0.117\end{array}$ & $\begin{array}{r}2.3 \\
-0.9 \\
5.9\end{array}$ & Orsat probe \\
\hline $\begin{array}{l}\text { Sunavala, } \\
{[19], 1957}\end{array}$ & $\begin{array}{l}\text { a1r/a1r } \\
\text { a1r/a1r }\end{array}$ & $\begin{array}{l}1.00 \\
0.50\end{array}$ & $\begin{array}{l}0.48- \\
0.95\end{array}$ & $\begin{array}{l}28,700- \\
56,900\end{array}$ & $\begin{array}{l}0.110 \\
0.108\end{array}$ & $\begin{array}{l}13.6 \\
14.0\end{array}$ & $\begin{array}{l}\text { tracer No IR } \\
\text { thermocouple }\end{array}$ \\
\hline $\begin{array}{l}\text { W11son, } \\
{[20], 1964}\end{array}$ & $\begin{array}{l}\text { a1r/a1r } \\
\text { a1r/a1r }\end{array}$ & $\begin{array}{l}0.94 \\
0.60\end{array}$ & $\begin{array}{l}1.27 \\
1.27\end{array}$ & $\begin{array}{l}20,000- \\
40,000\end{array}$ & $\begin{array}{l}0.088 \\
0.088\end{array}$ & $\begin{array}{l}0.0 \\
0.0\end{array}$ & $\begin{array}{l}\text { resistance } \\
\text { thermometry }\end{array}$ \\
\hline $\begin{array}{l}\text { Becker, } \\
{[9], 1967}\end{array}$ & $a 1 r / a 1 r$ & 1.00 & 0.64 & 54,000 & 0.093 & 4.8 & nephelometry \\
\hline $\begin{array}{l}\text { McQua1d, } \\
{[37], 1974}\end{array}$ & $\begin{array}{l}\mathrm{Ar} / \mathrm{aIr} \\
\mathrm{CO}_{2} / \mathrm{aIr}\end{array}$ & $\begin{array}{l}1.38 \\
1.53\end{array}$ & $\begin{array}{l}1.27 \\
1.27\end{array}$ & $\begin{array}{l}10,000 \\
10,000\end{array}$ & $\begin{array}{l}0.115 \\
0.121\end{array}$ & $\begin{array}{l}-0.1 \\
-0.1\end{array}$ & hot-wire \\
\hline $\begin{array}{l}\text { Chassa1ng, } \\
{[38], 1976 *}\end{array}$ & $\mathrm{CO}_{2} / \mathrm{a} 1 \mathrm{r}$ & 1.53 & 4.00 & 26,500 & 0.086 & 0.8 & Infrared \\
\hline $\begin{array}{l}\text { Ebrah1m1, } \\
{[15], 1977}\end{array}$ & $a 1 r / a 1 r$ & 1.00 & 0.80 & 15,400 & 0.115 & 0.0 & nephelowetry \\
\hline $\begin{array}{l}\text { B1rch, }[10], \\
1978\end{array}$ & $\begin{array}{l}\text { natural } \\
\text { gas/a1r }\end{array}$ & 0.56 & 1.27 & 16,000 & 0.125 & 11.6 & Raman \\
\hline $\begin{array}{l}\text { Lockwood, } \\
{[39], 1980^{*}}\end{array}$ & $a 1 r / a 1 r$ & 0.54 & 1.93 & 50,400 & 0.092 & 5.5 & thermocouple \\
\hline $\begin{array}{l}\text { B1 rch, }[40], \\
1984\end{array}$ & $\begin{array}{l}\text { natural } \\
\text { gas/a1r } \\
\mathrm{C}_{2} \mathrm{H}_{4} / \mathrm{aIr}\end{array}$ & $\begin{array}{l}0.56 \\
0.97\end{array}$ & $\begin{array}{l}0.27 \\
0.27\end{array}$ & - & $\begin{array}{l}0.102 \\
0.102\end{array}$ & - & $\begin{array}{l}\text { gas } \\
\text { chroma togr aphy }\end{array}$ \\
\hline $\begin{array}{l}\text { N1wa, } \\
1984\end{array}$ & $\begin{array}{l}\mathrm{He} / \mathrm{alr} \\
\mathrm{CO}_{2} / \mathrm{alr} \\
\mathrm{CF}_{2} \mathrm{Cl}_{2} / \mathrm{alr}\end{array}$ & $\begin{array}{l}0.14 \\
1.53 \\
4.30\end{array}$ & $\begin{array}{l}0.07 \\
0.07 \\
0.07\end{array}$ & $\begin{array}{r}1,300 \\
5,000 \\
10,000\end{array}$ & $\begin{array}{l}0.156 \\
0.114 \\
0.104\end{array}$ & $\begin{array}{l}- \\
-\end{array}$ & Rayle1gh \\
\hline $\begin{array}{l}\text { P1tts, }[1,2] \text {, } \\
1984\end{array}$ & $\mathrm{CH}_{4} / \mathrm{a1T}$ & 0.55 & 0.64 & 4,000 & 0.112 & -2.0 & Rayle1gh \\
\hline $\begin{array}{l}\text { Dahm, }[16] \text {, } \\
1985\end{array}$ & $\mathrm{H}_{2} \mathrm{O} / \mathrm{H}_{2} \mathrm{O}$ & 1.00 & 0.25 & 5,000 & 0.093 & - & Absorption \\
\hline $\begin{array}{l}\text { Schefer } \\
{[42], 1985}\end{array}$ & $\mathrm{C}_{3} \mathrm{H}_{8} / \mathrm{a} 1 \mathrm{r}$ & 1.53 & 0.50 & 68,000 & 0.093 & 6.0 & Rayle1gh \\
\hline
\end{tabular}


Table 9. Literature asurements of Centerline Unmixedness in Axis ymmetric Turbulent Jets

\begin{tabular}{|c|c|c|c|c|c|c|}
\hline $\begin{array}{l}\text { P1rst Author } \\
\text { Ref., Date }\end{array}$ & Jet/Coflow & $P_{0} / D_{-}$ & $\operatorname{Re}$ & $\begin{array}{c}\text { Asymptote } \\
z / r_{0}\end{array}$ & $\begin{array}{l}\text { Maximum } \\
\mathrm{C}_{\mathrm{m}} / \overline{\mathrm{C}}_{\mathrm{w}}\end{array}$ & $\begin{array}{c}\text { Maximum } \\
z / r_{0}\end{array}$ \\
\hline $\begin{array}{l}\text { Corrsind, } \\
{[6], 1949}\end{array}$ & a1r/a1r & 0.66 & - & none & 0.19 & 54 \\
\hline $\begin{array}{l}\text { Rosenwe 18 } \\
{[46], 1961}\end{array}$ & alr/a1t & 1.00 & 26,200 & none & 0.21 & 67 \\
\hline $\begin{array}{l}\text { W1180n, } \\
{[20], 1964}\end{array}$ & a1r/air & 0.62 & $>20,000$ & 90 & 0.18 & 200 \\
\hline $\begin{array}{l}\text { Becker, } \\
{[9], 1967}\end{array}$ & alr/alr & 1.00 & 54,000 & none & 0.21 & 128 \\
\hline $\begin{array}{l}\text { Way }^{2},[47], \\
1971\end{array}$ & $\begin{array}{l}10 \% \mathrm{He} / \mathrm{alr} \\
\mathrm{He} / \mathrm{alr}\end{array}$ & $\begin{array}{l}0.91 \\
0.14\end{array}$ & $\begin{array}{l}7,170 \\
3,290\end{array}$ & $\begin{array}{c}30 \\
\text { none }\end{array}$ & $\begin{array}{l}0.24 \\
0.37\end{array}$ & $\begin{array}{l}40 \\
40\end{array}$ \\
\hline $\begin{array}{l}\text { McQuald, } \\
{[37], 1974}\end{array}$ & Ar/air & 1.38 & 10,000 & 34 & 0.36 & 40 \\
\hline $\begin{array}{l}\text { Ebrah1m1, } \\
{[15], 1977}\end{array}$ & a1r/a1r & 1.00 & 15,400 & 80 & 0.25 & 100 \\
\hline $\begin{array}{l}\text { B1rch, }[10], \\
1978\end{array}$ & $\begin{array}{l}\text { natural } \\
\text { gas/air }\end{array}$ & 0.56 & 16,000 & none & 0.28 & 140 \\
\hline $\begin{array}{l}\text { Lockwood, } \\
{[39], 1980}\end{array}$ & a1r/a1r & 0.53 & 50,400 & 73 & 0.21 & 92 \\
\hline $\begin{array}{l}\text { Nakamura } \\
{[48], 1982}\end{array}$ & $\mathrm{H}_{2} \mathrm{O} / \mathrm{H}_{2} \mathrm{O}$ & 1.00 & 5,000 & 200 & 0.21 & 600 \\
\hline $\begin{array}{l}\text { N1wa, [41], } \\
1984\end{array}$ & $\begin{array}{l}\mathrm{He} / \mathrm{alr} \\
\mathrm{CO}_{2} / \mathrm{alt} \\
\mathrm{Cr}_{2} \mathrm{Cl}_{2} / \mathrm{alr}\end{array}$ & $\begin{array}{l}0.14 \\
1.53 \\
4.30\end{array}$ & $\begin{array}{r}1,300 \\
5,000 \\
10,000\end{array}$ & $\begin{array}{c}\text { none } \\
90 \\
\text { none }\end{array}$ & $\begin{array}{l}0.20 \\
0.20 \\
0.20\end{array}$ & $\begin{array}{l}160 \\
160 \\
160\end{array}$ \\
\hline $\begin{array}{l}\text { Pites, }[1,2] \\
1984\end{array}$ & $\mathrm{CH}_{4} / \mathrm{a} 1 \mathrm{r}$ & 0.55 & 4,000 & none & 0.23 & 63 \\
\hline $\begin{array}{l}\text { Dahm, }[16] \text {, } \\
1985\end{array}$ & $\mathrm{H}_{2} \mathrm{O} / \mathrm{H}_{2} \mathrm{O}$ & 1.00 & 5,000 & Done & 0.30 & 600 \\
\hline $\begin{array}{l}\text { Schefer, } \\
{[42], 1985}\end{array}$ & $\mathrm{C}_{3} \mathrm{H}_{8} / \mathrm{alt}$ & 1.53 & 68,000 & none & 0.25 & 123 \\
\hline
\end{tabular}

Experimental data for unmixedness have a maximum and then falloff as a function of downstream distance.

2 Measurements are reported for only two downstream positions. 
Table 10. Centarline Unmixedness Results for This Study

\begin{tabular}{|c|c|c|c|c|c|}
\hline Jet/Coflow & $0_{0} / p_{0}$ & $\operatorname{Re}$ & $\begin{array}{c}\text { As ymp tote } \\
z / r_{0}\end{array}$ & $\begin{array}{c}\text { Maximum } \\
\mathrm{C}_{\mathrm{m}}^{\prime} / \overline{\mathrm{C}}_{\mathrm{m}}\end{array}$ & $\begin{array}{c}\text { Maximum } \\
z / r_{0}\end{array}$ \\
\hline $\begin{array}{l}\text { He/air } \\
\text { free He/air }\end{array}$ & $\begin{array}{l}0.14 \\
0.14\end{array}$ & $\begin{array}{l}3,950 \\
3,950\end{array}$ & $\begin{array}{r}23 \\
40\end{array}$ & $\begin{array}{l}0.23 \\
0.26\end{array}$ & $\begin{array}{r}63 \\
110\end{array}$ \\
\hline $\mathrm{CH}_{4} / \mathrm{air}$ & 0.55 & 3,950 & 20 & 0.23 & 63 \\
\hline $\mathrm{C}_{3} \mathrm{H}_{8} / \mathrm{CO}_{2}$ & 1.192 & 3,960 & 23 & 0.23 & 63 \\
\hline $\begin{array}{l}\mathrm{C}_{3} \mathrm{H}_{8} / \text { air } \\
\mathrm{C}_{3} \mathrm{H}_{8} / \text { air }\end{array}$ & $\begin{array}{l}1.55 \\
1.55\end{array}$ & $\begin{array}{l}3,960 \\
7,930\end{array}$ & $\begin{array}{l}28 \\
50\end{array}$ & $\begin{array}{l}0.23 \\
0.23\end{array}$ & $\begin{array}{l}63 \\
63\end{array}$ \\
\hline $\mathrm{C}_{3} \mathrm{H}_{8} / \mathrm{air}$ & 1.55 & 11,880 & none & 0.22 & 63 \\
\hline $\mathrm{CF}_{4} / \mathrm{air}$ & 3.00 & 3,950 & 50 & 0.23 & 63 \\
\hline $\mathrm{CF}_{4} / \mathrm{air}$ & 3.00 & $i .390$ & none & 0.22 & 63 \\
\hline $\begin{array}{l}\mathrm{SF}_{6} / \mathrm{air} \\
\mathrm{SF}_{6} / \mathrm{air}\end{array}$ & $\begin{array}{l}5.11 \\
5.11\end{array}$ & $\begin{array}{l}7,890 \\
11,960\end{array}$ & $\begin{array}{l}\text { none } \\
\text { none }\end{array}$ & $\begin{array}{l}0.19 \\
0.19\end{array}$ & $\begin{array}{l}63 \\
63\end{array}$ \\
\hline
\end{tabular}

The unmixedness vaiues for this flow fall with increasing downstream distance (see fig. 28 and disc'sssion in text). 
Figure 1. A schematic representation of the three flow regimes of an axisymmetric, constant-density, turbulent jet is shown. Representative radial profiles of velocity and jet fluid concentration are shown at one location in the potential core portion of the flow and at three positions in the selfsimilar regime.

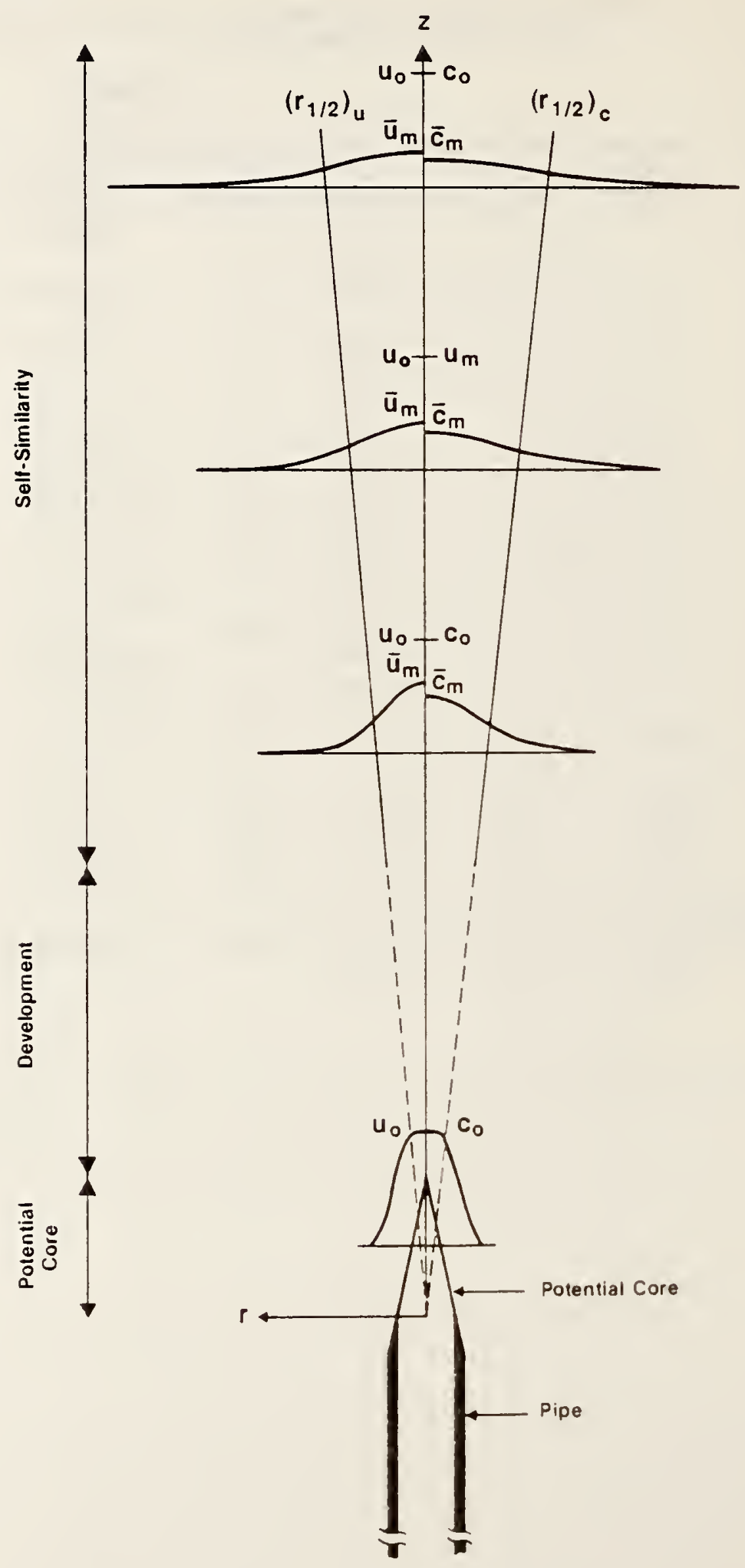




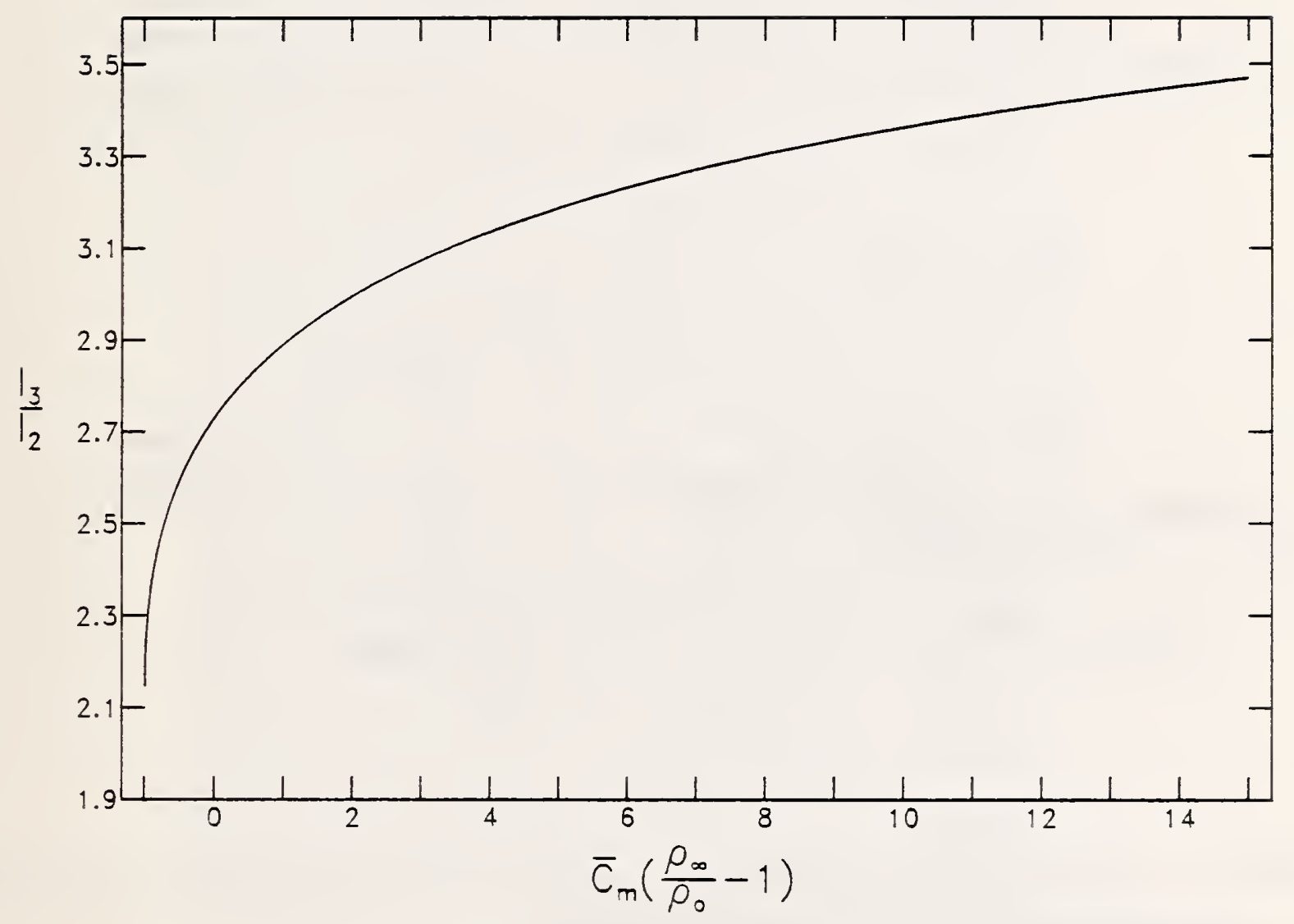

Figure 2. Calculated values for the ratio of the integrals $I_{3}$ (eq. 44 ) and $I_{2}$ (eq. (43)) are plotted as a function of $\overrightarrow{\mathrm{C}}_{\mathrm{m}}\left(\left(0_{\infty} / 0_{0}\right)-1\right)$. 


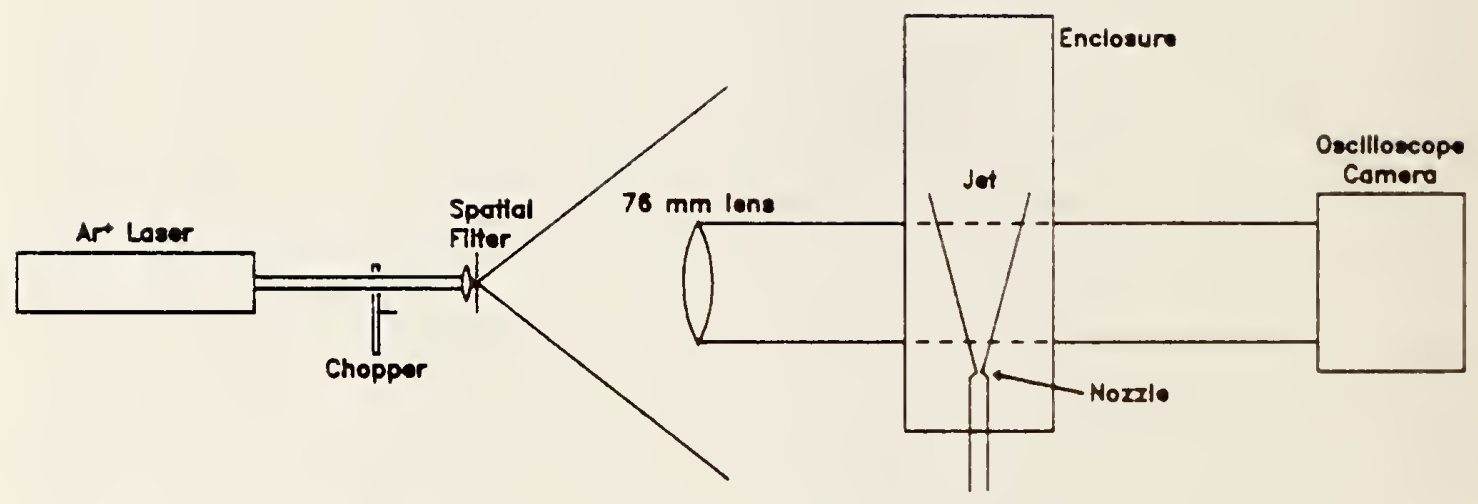

Figure 3. The experimental arrangement emploved for time-resolved shadowgraphy of variable density flows is shown. 


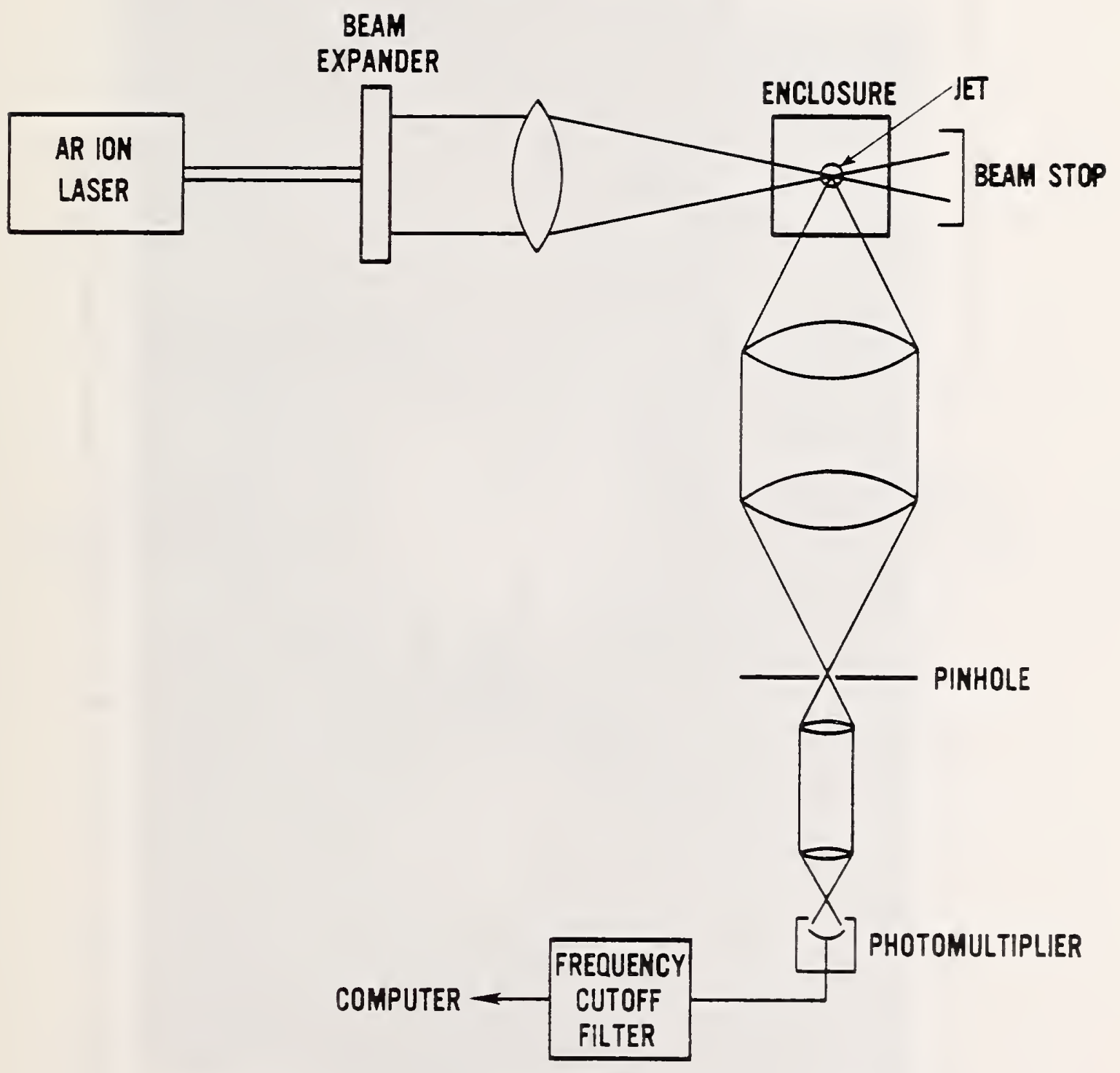

Figure 4. This figure shows the experimental system for making real-time, spatially-resolved, concentration measurements in turbulent flows of binary gas mixtures. 


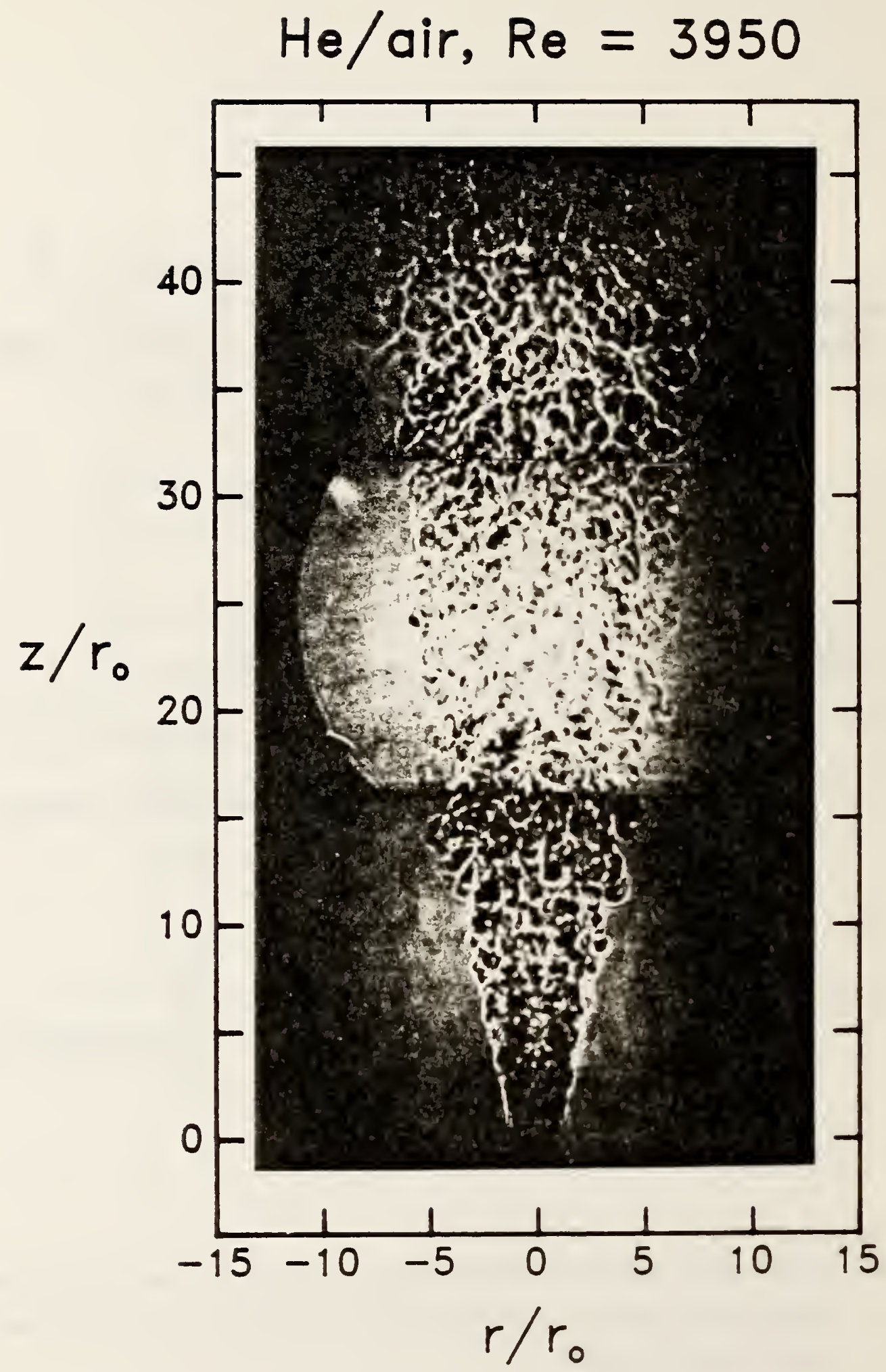

Figure 5. Three shadowgraphs of a turbulent helium jet flowing into a slow coflow of air are superimposed. The Reynolds number for the jet is 3950. 


\section{Free $\mathrm{He} / \mathrm{air}, \operatorname{Re}=3950$}

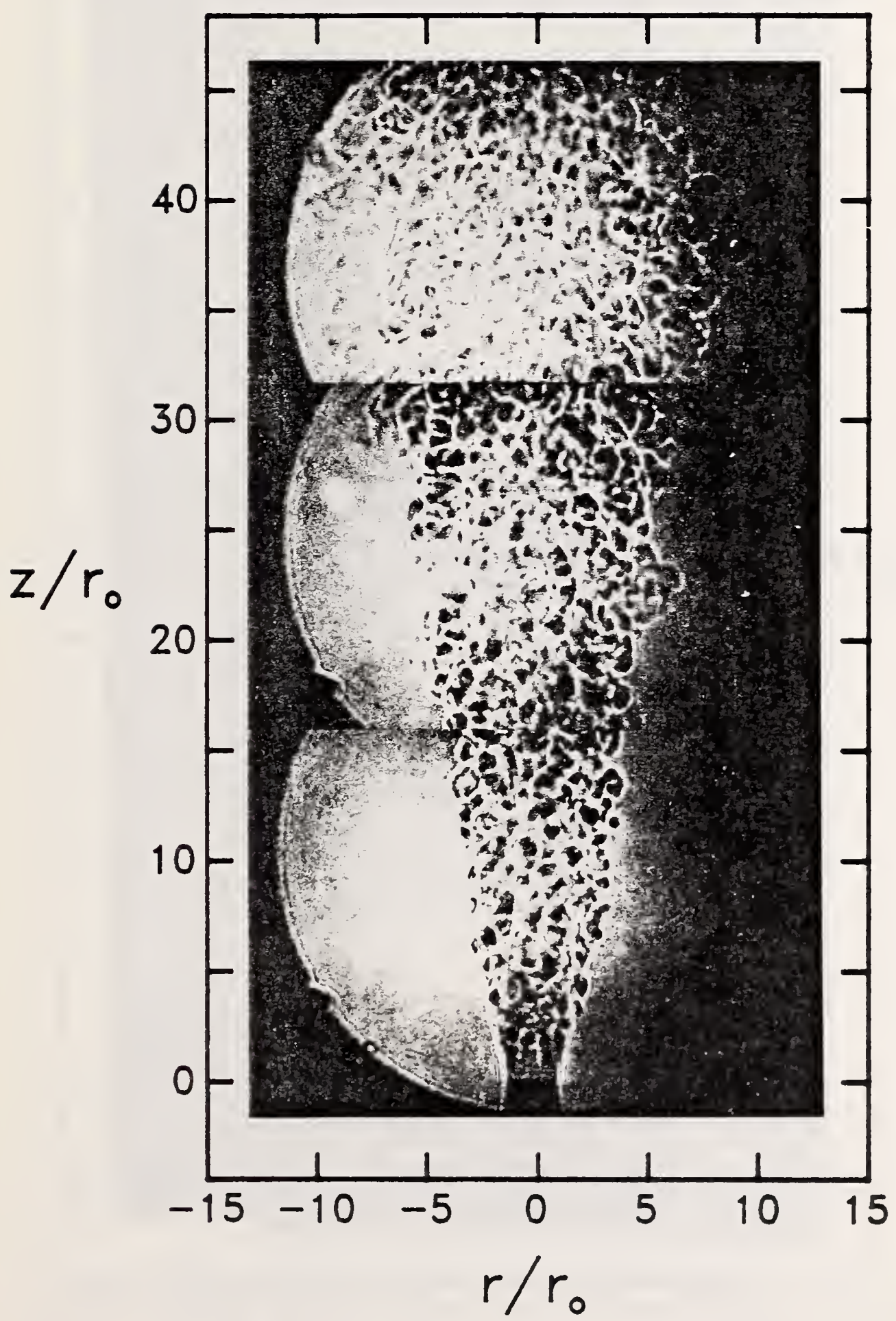

Figure 5. Superimposed shadowgraphs of the same helium jet visualized in fig. 5 are shown, but in this case, there is no coflow and the surroundings are the ambient air. 
Figure 7. Four superimposed shadowgraphs are reproduced for a turbulent methane jet at a Reynolds number of 3950 entering a slow coflow of air.

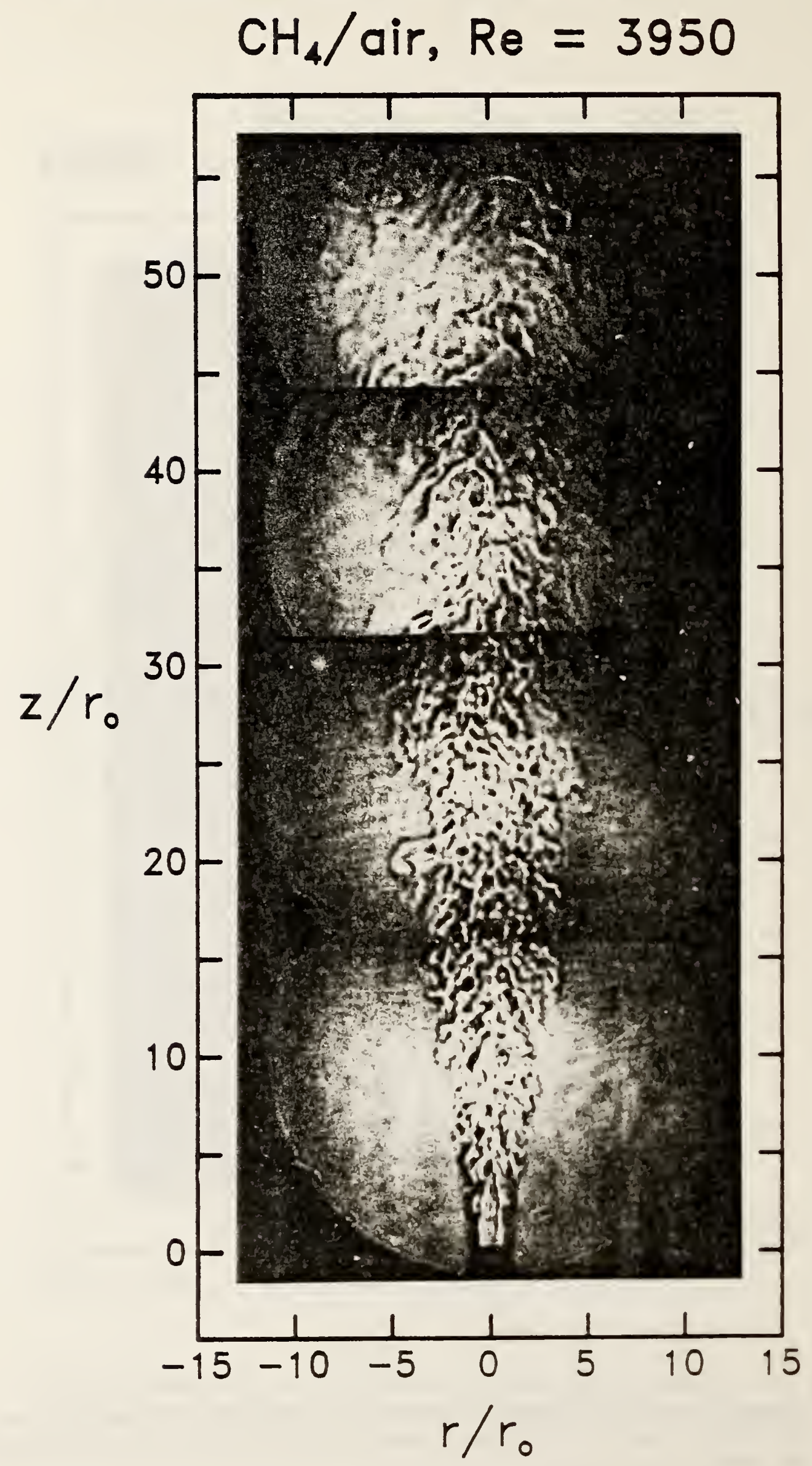


Figure 8. The superposition of four shadowgraphs is shown for a turbulent jet of propane flowing into a slow coflow of carbon dioxide. The Reynolds number for the jet is 3960 .

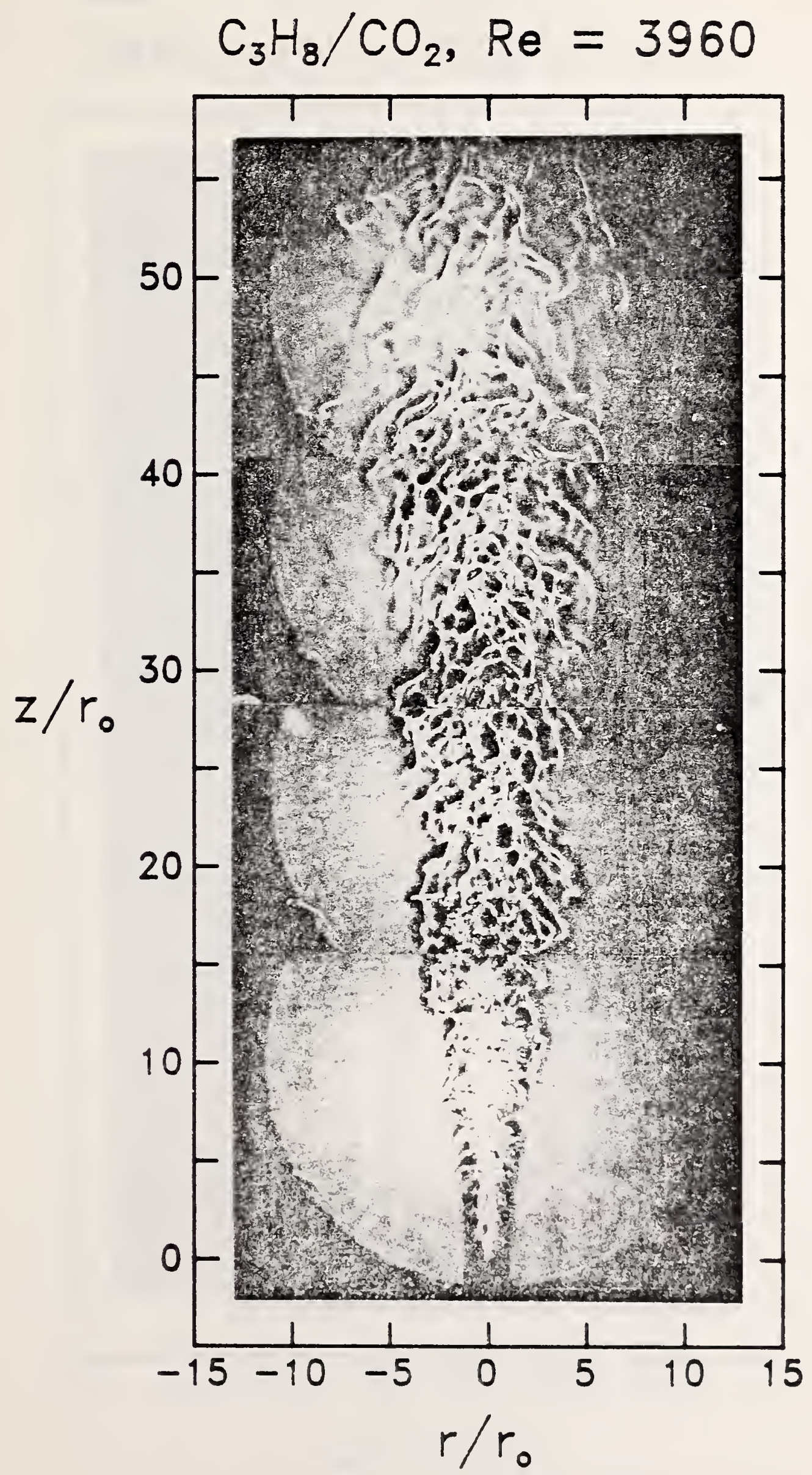


Figure 9. Shadowgraphs of the same propane jet visualized in fig. 8, but flowing into a slow coflow of air, are shown.

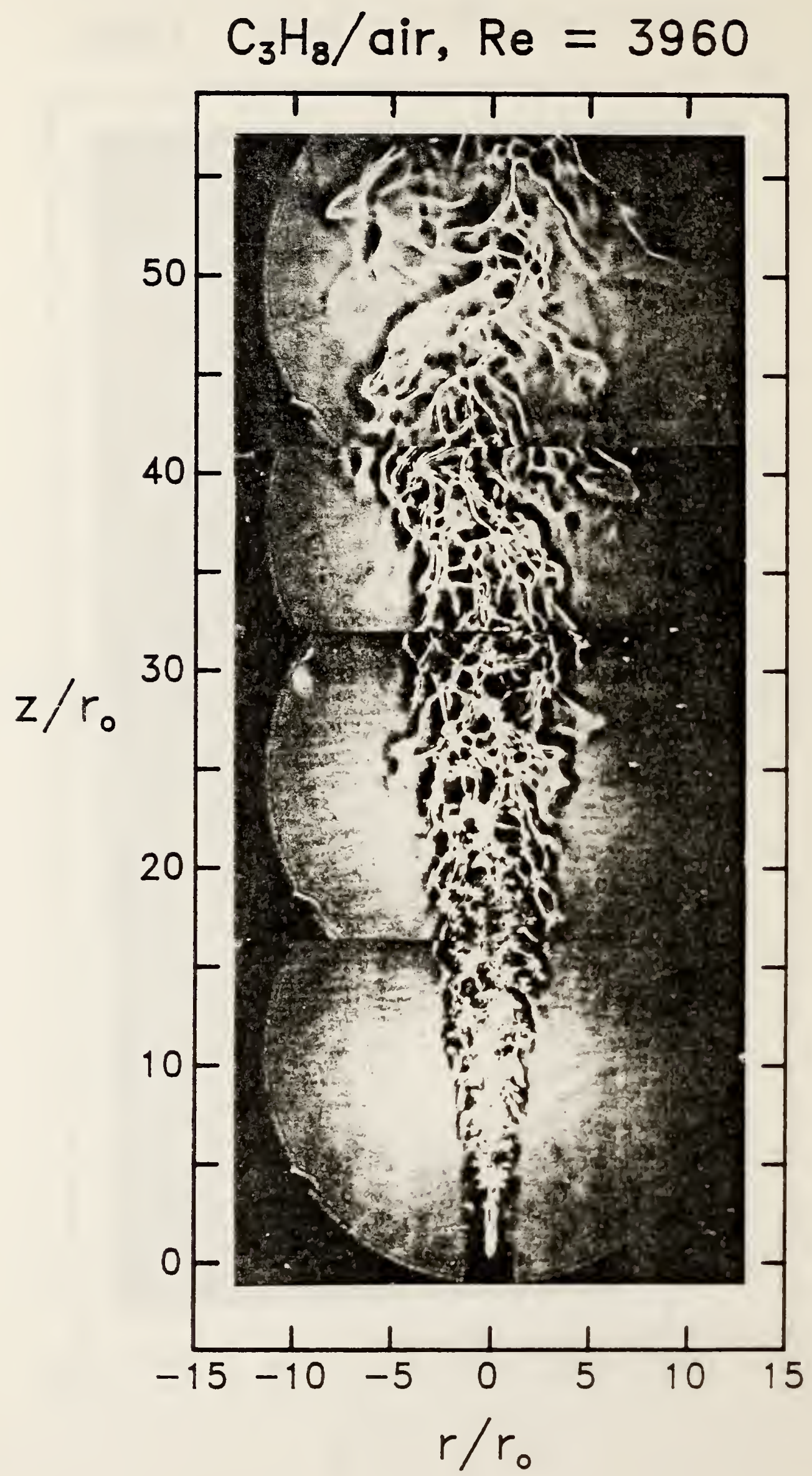


Figure 10. The Reynolds number of the propane jet shown in fig. 9 has been increased to 7930 for the four shadowgraphs which have been superimposed. The coflow gas is again air.

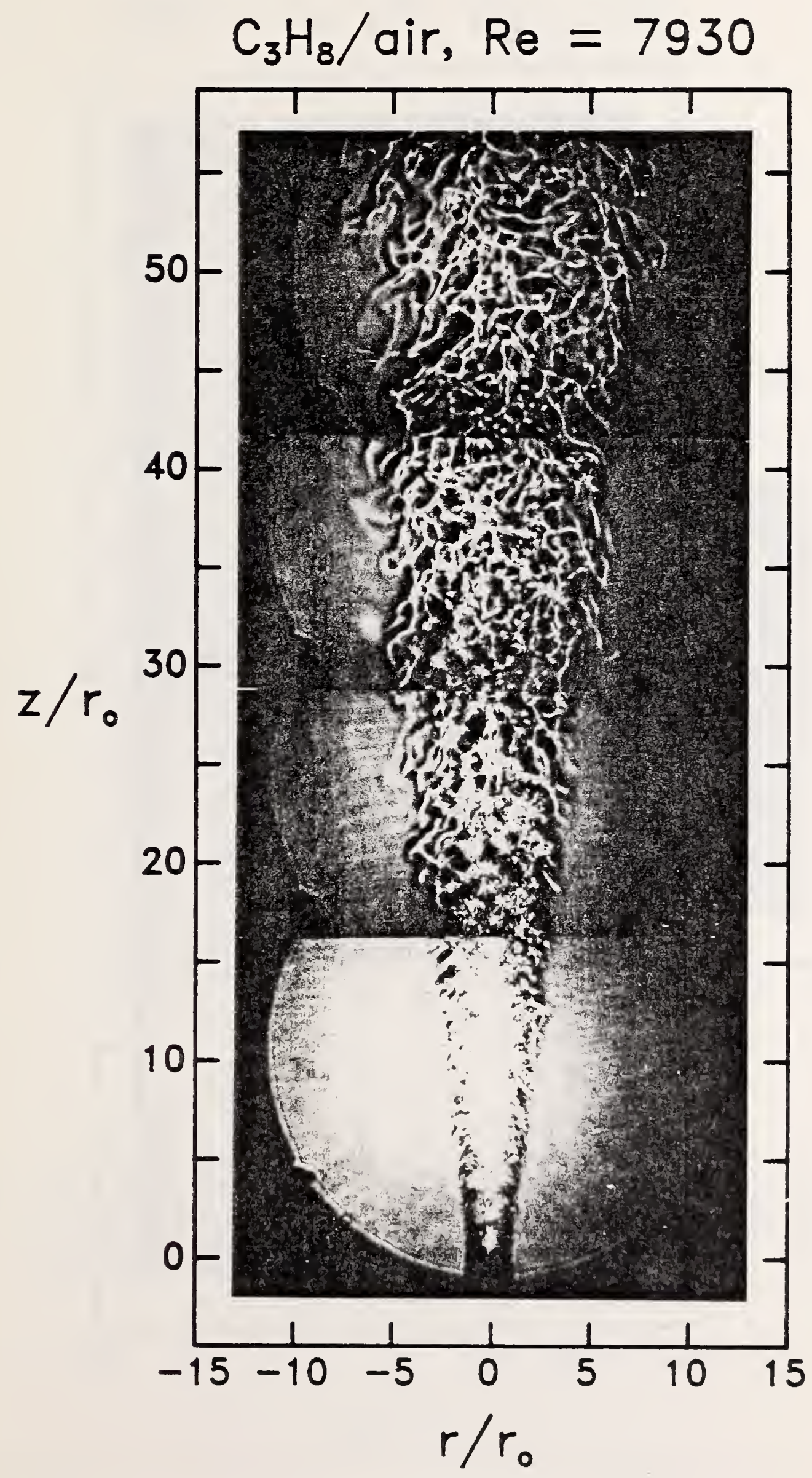


Figure 11. Four superimposed shadowgraphs are shiown for a turbulent jet of propane $(\operatorname{Re}=11,880)$ entering a slow coflow of air.

\section{$\mathrm{C}_{3} \mathrm{H}_{8} /$ air, $\mathrm{Re}=11880$}

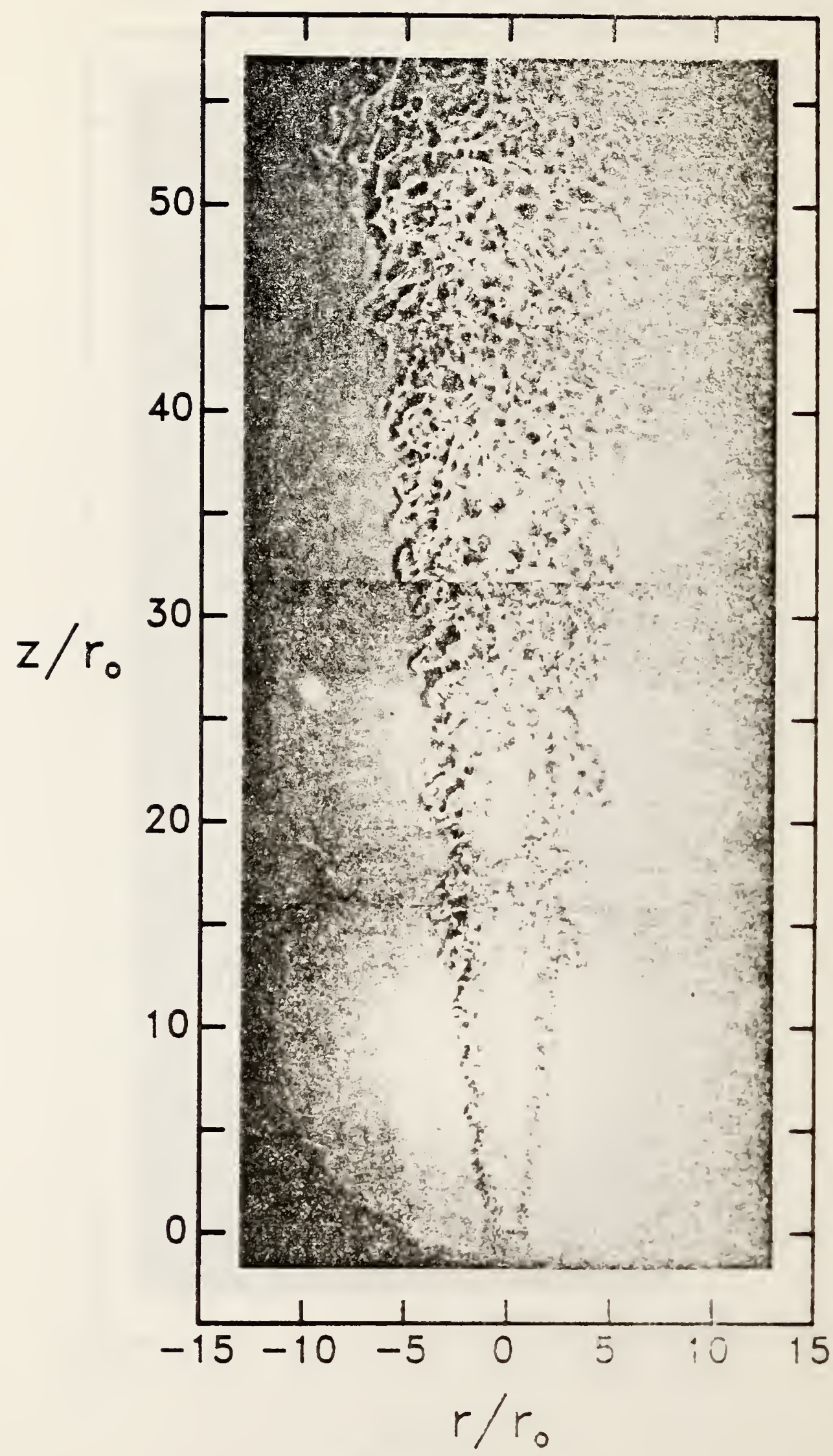




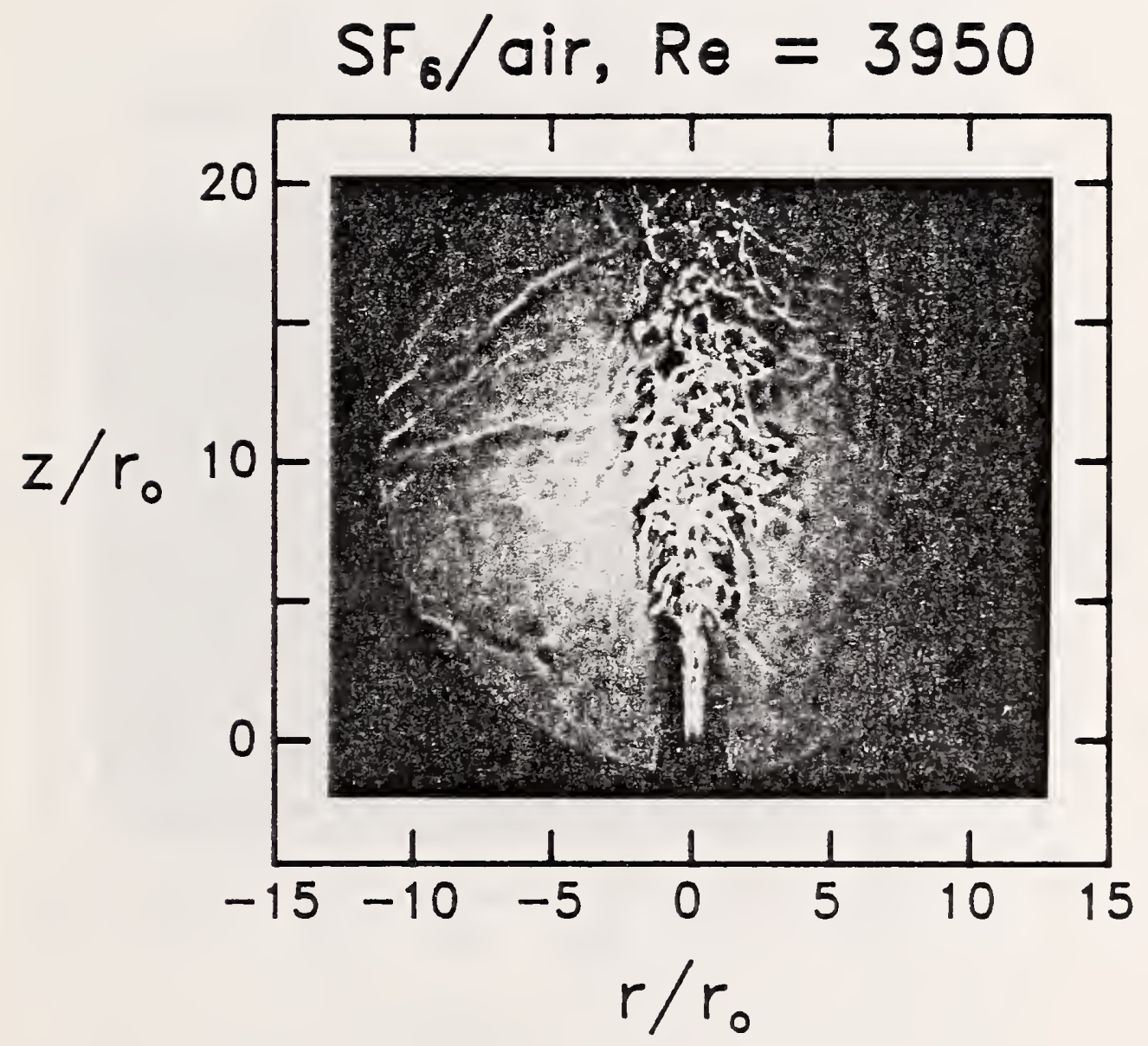

Figure 12. The near-field region of a sulfur hexafluoride turbulent jet $(\operatorname{Re}=3950)$ is visualized by shadowgraphy. Note the presence of jet fluid in regions well outside of the jet. 


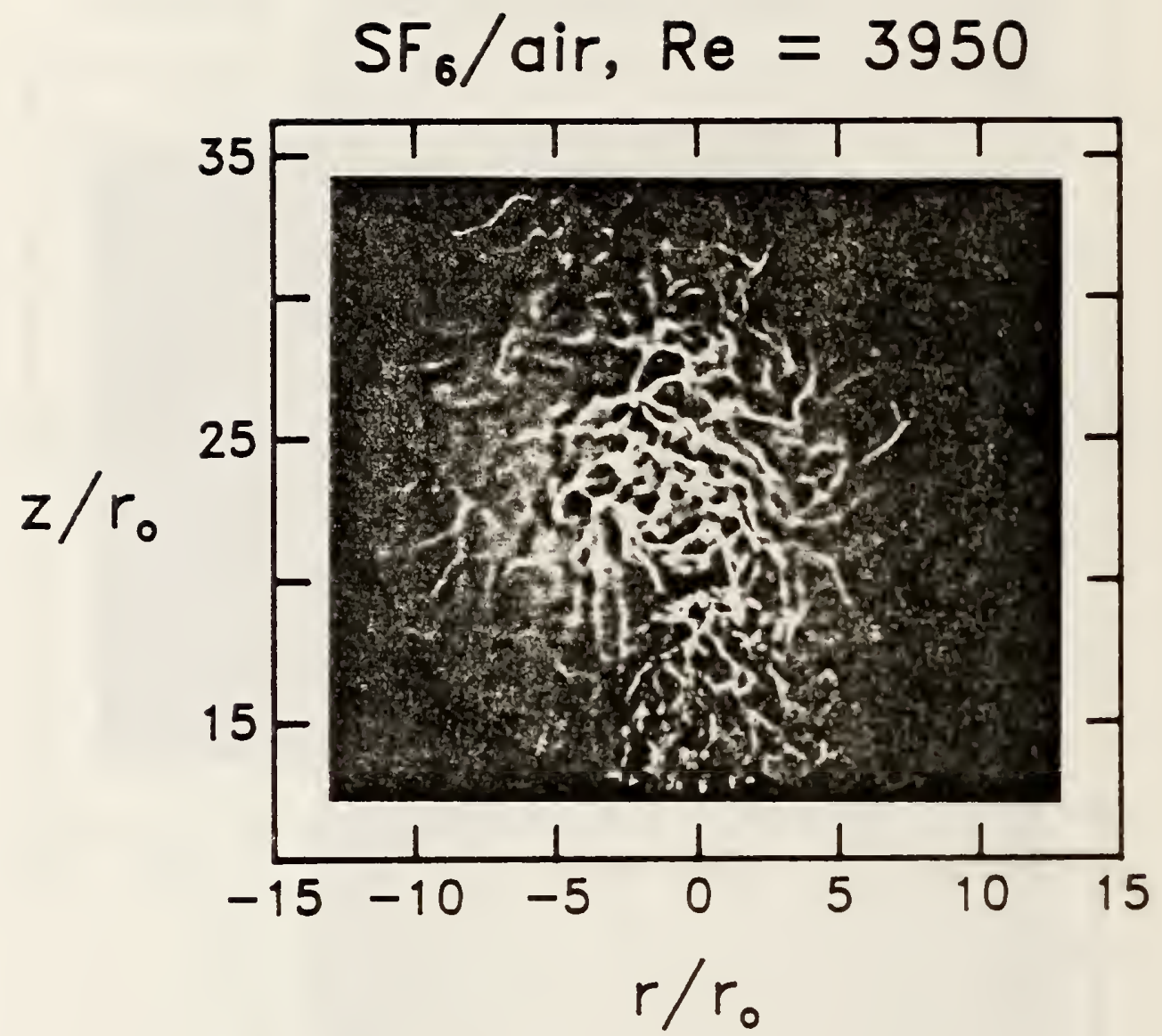

Figure 13. A shadowgraph for the same flow described in fig. 12 is shown at larger downstream distances. Again, jet gas is observed in locations well outside of the jet. 


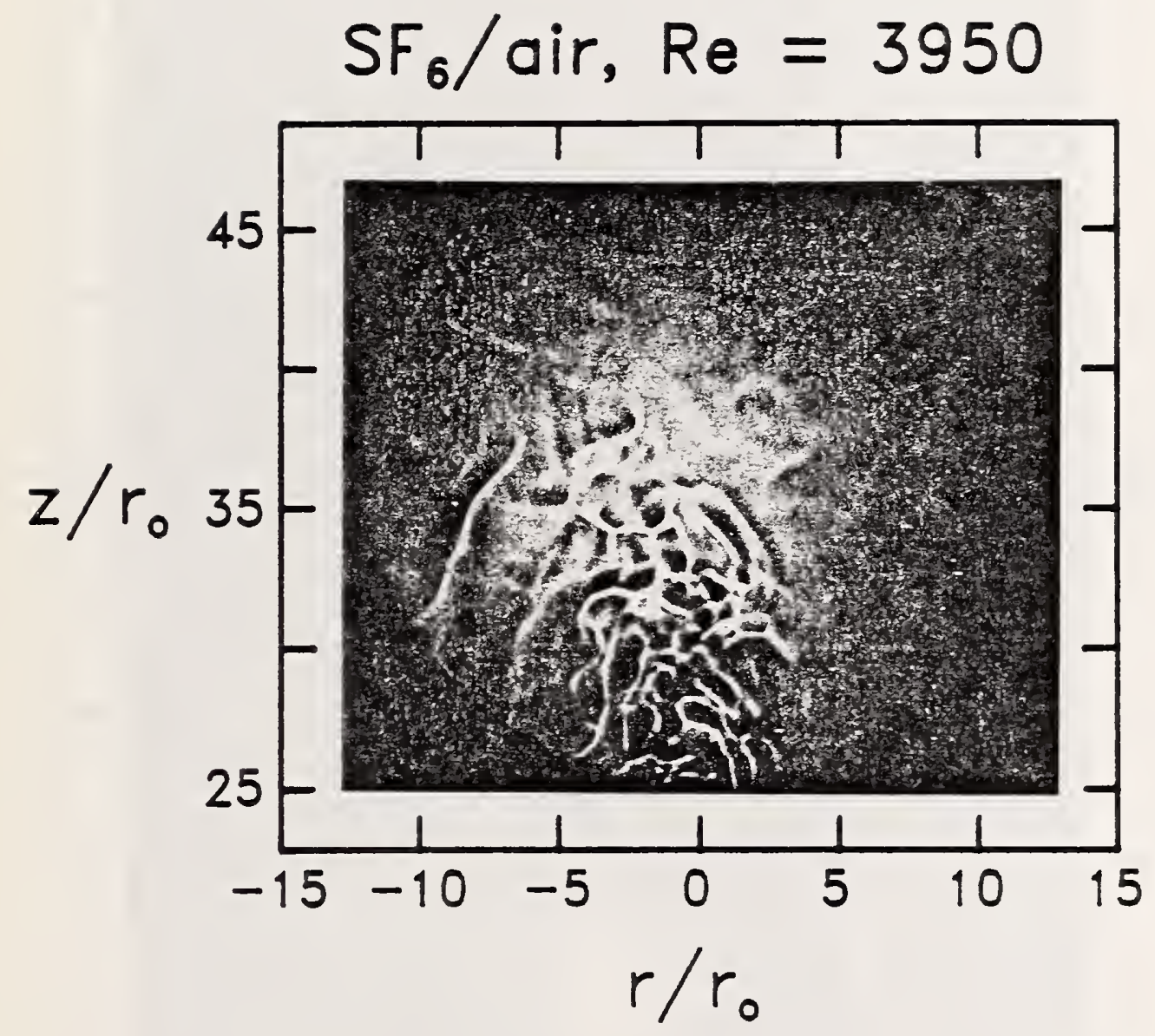

Figure 14. The formation of a fountain by the strongly negatively buoyant jet of $\mathrm{SF}_{6}$ (Re = 3950) flowing into a coflow of air is visualized by shadowgraphy. The range of downstream distances extends from $z / r_{0}=25$ to 47 . 
Figure 15. Four superimposed shadowgraphs of a turbulent fet of sulfur hexafluorlde ( $\operatorname{Re}=7890$ ) entering a slow coflow of alr are shown.

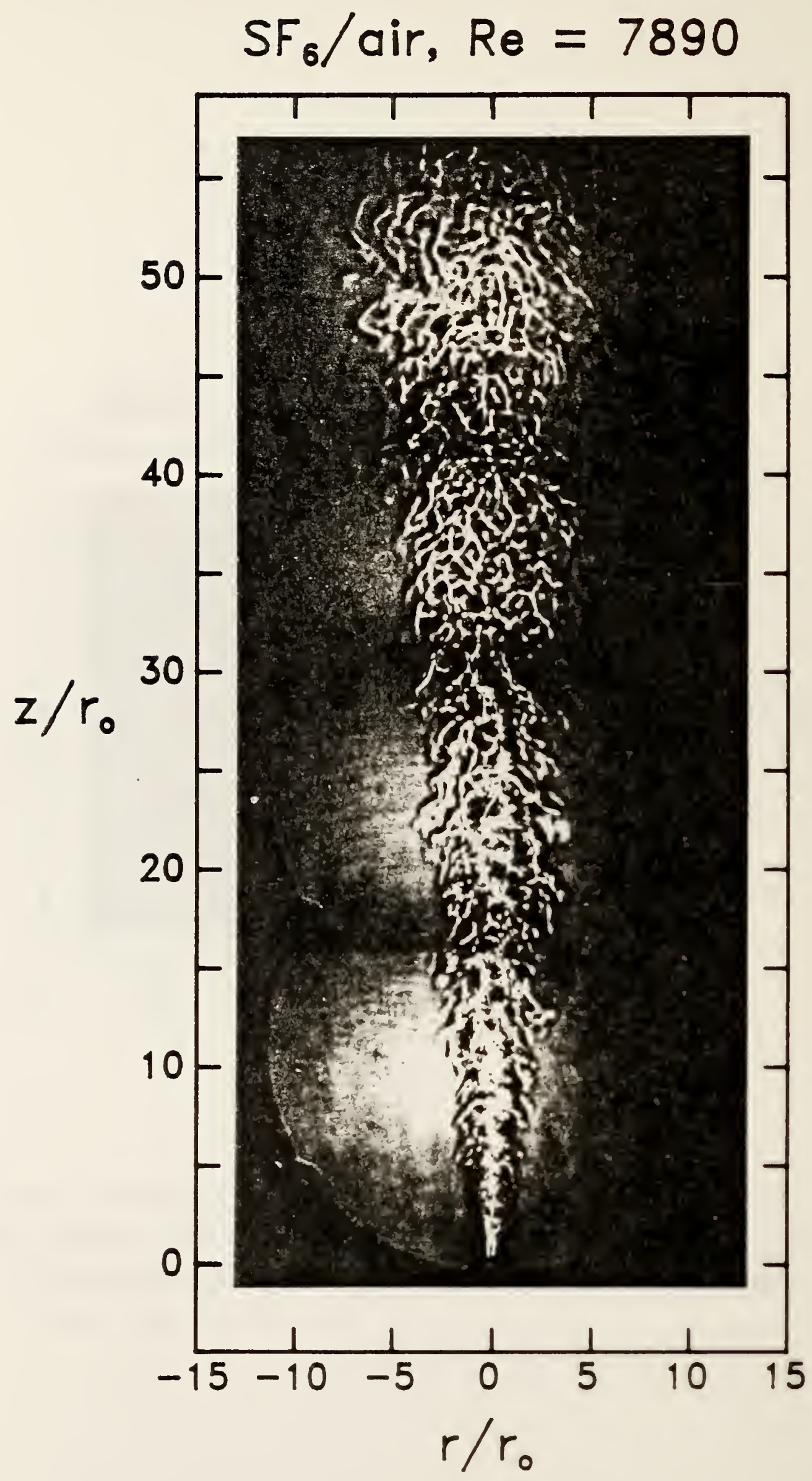


Figure 16. The Reynolds number has been increased to 11,860 for the sulfur hexafluoride turbulent jet entering a slow coflow of air.

\section{$S_{6} /$ air, $\operatorname{Re}=11860$}

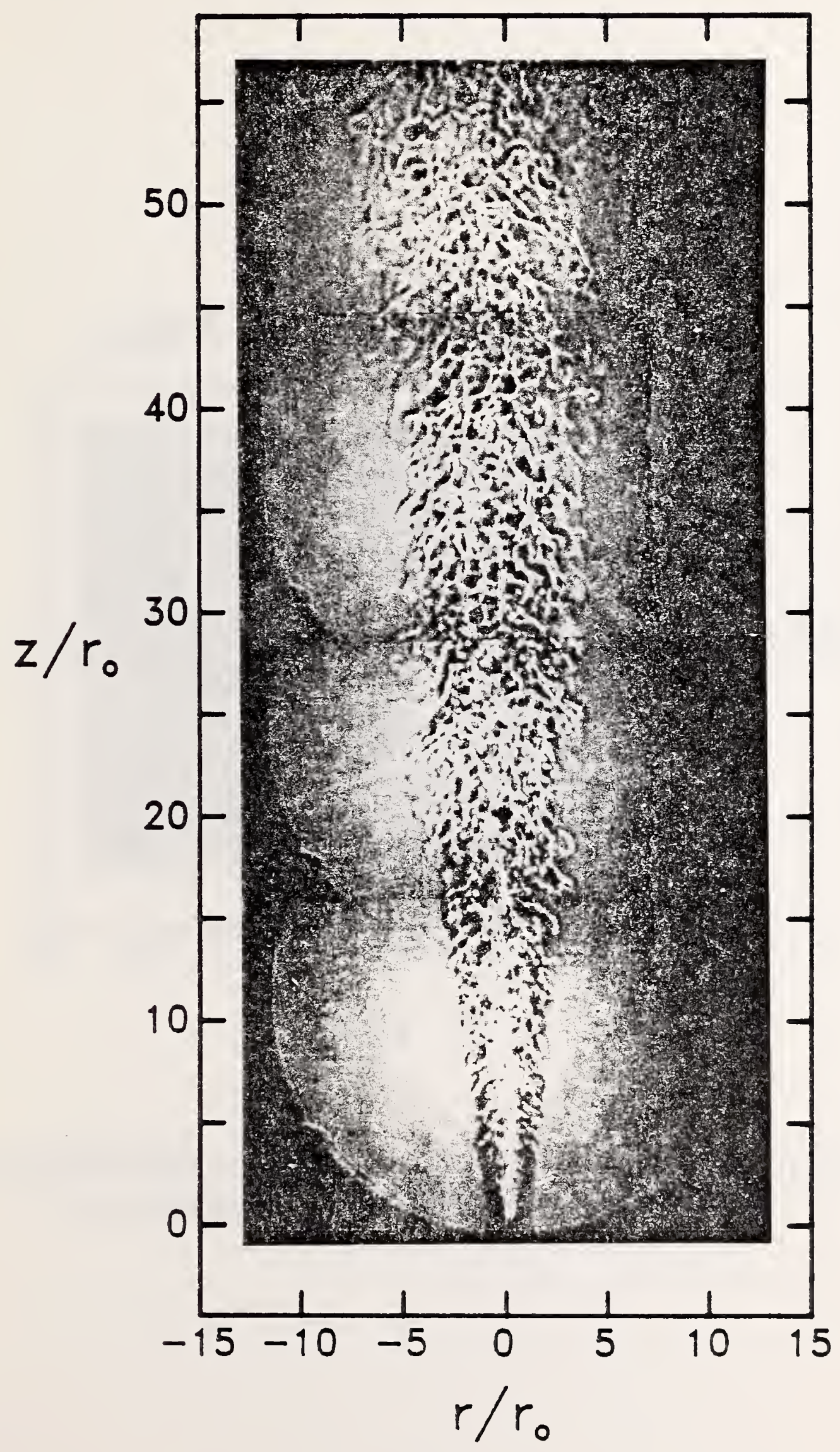




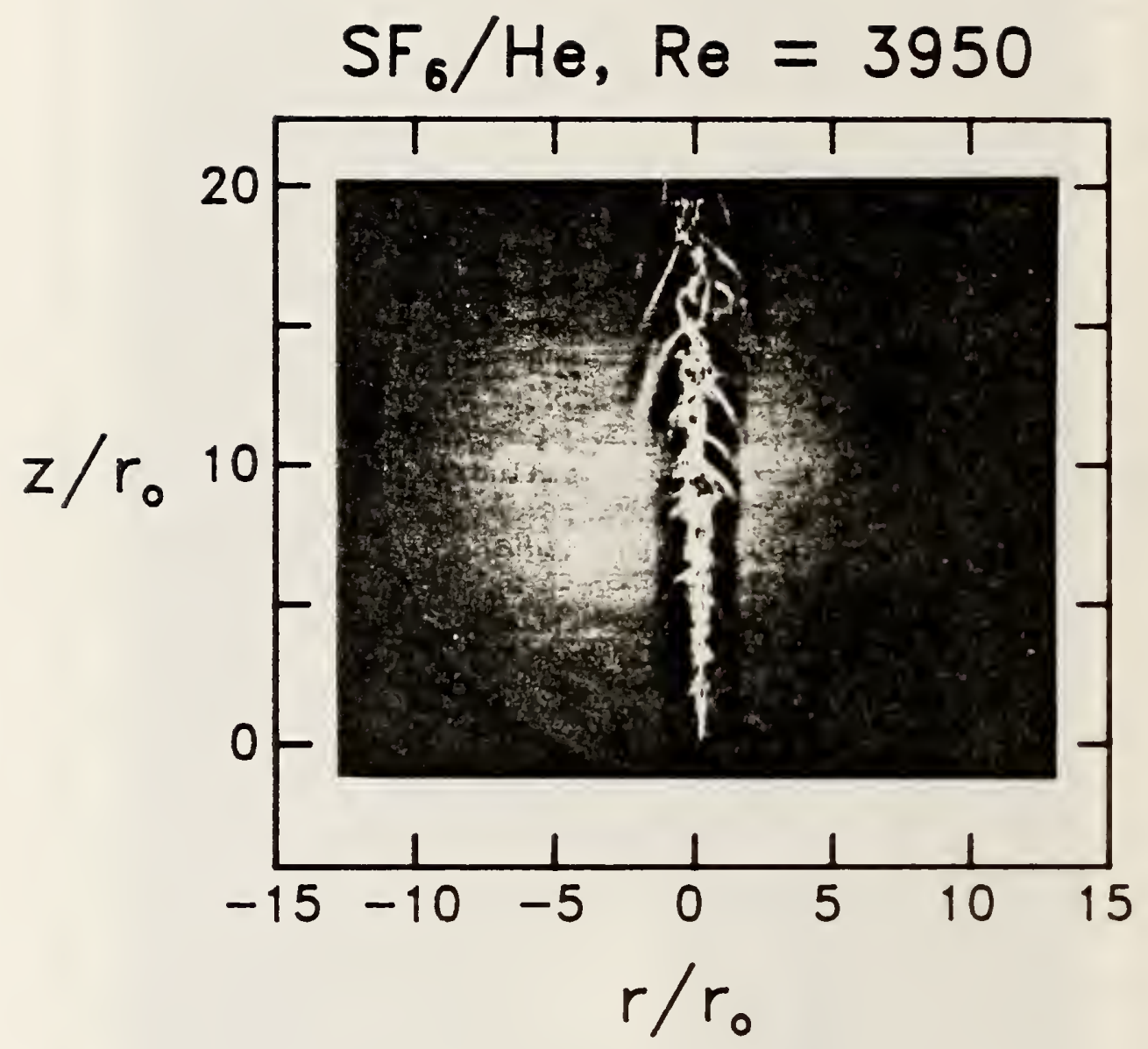

Figure 17. The near field region of a $S_{6}$ fet ( $\left.R e=3950\right)$ flowing into a slow coflow of helium is visualized by shadowgraphy. 


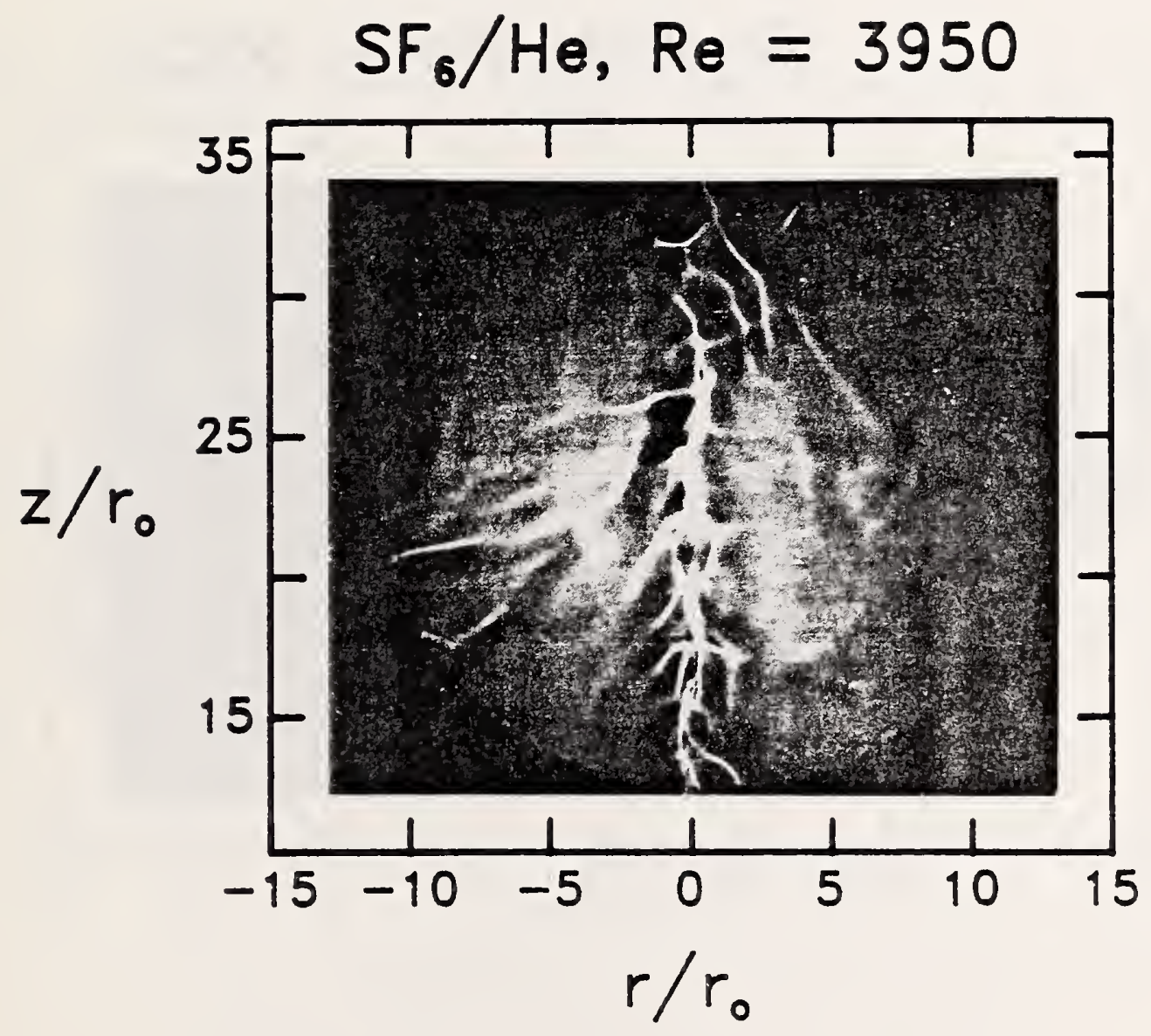

Figure 18. A shadowgraph centered at $z / r_{0} \simeq 24$ is shown for the $S F_{6}$ jet of $\operatorname{Re}=$ 3950 flowing into a slow coflow of helium. 


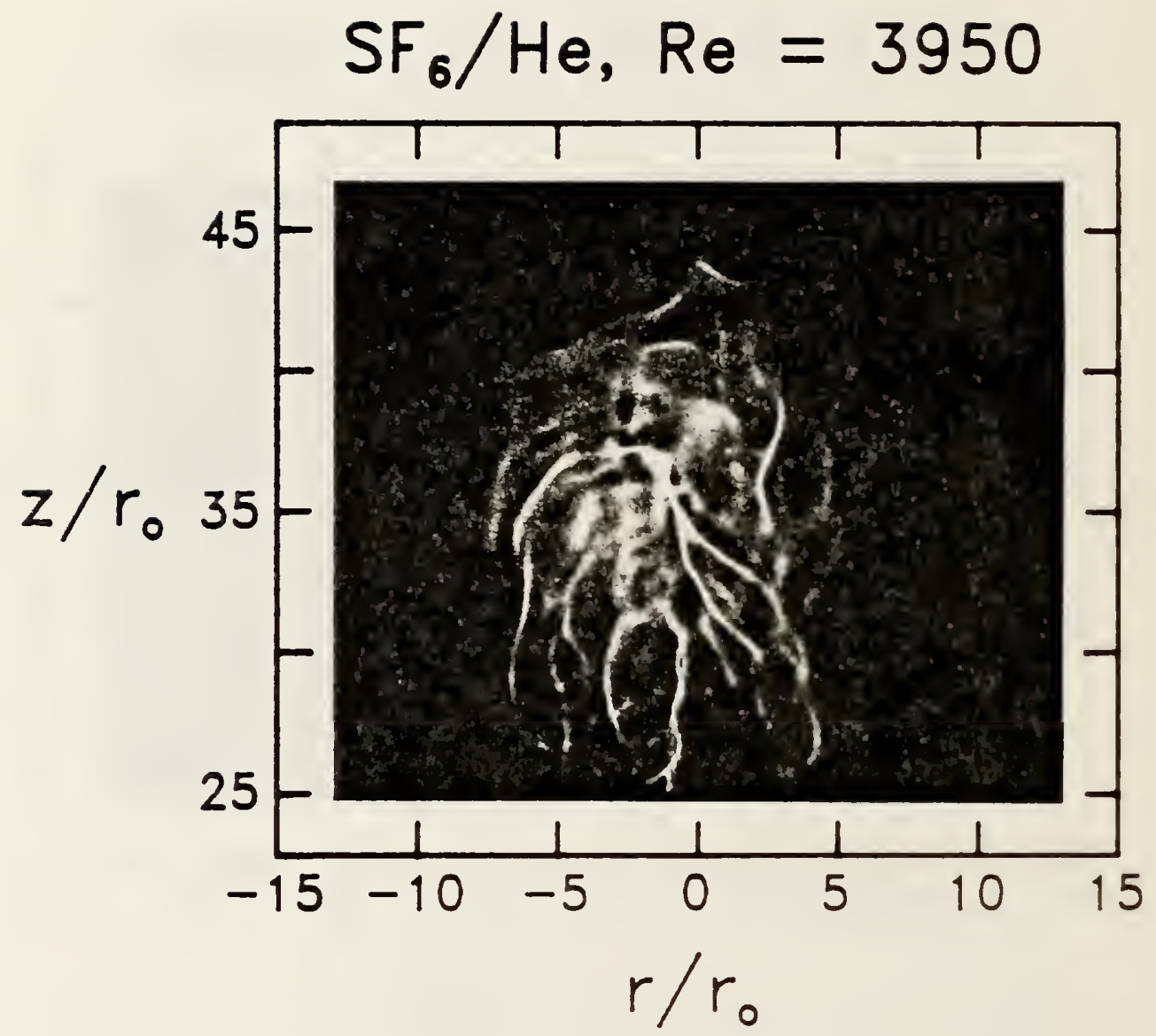

Figure 19. The fountain formed by the $\mathrm{SF}_{6}$ jet ( $\mathrm{Re}=3950$ ) entering into hellum can be clearly seen in this shadowgraph recorded for downstream distances extending from $z / r_{0}=25$ to 47 . 


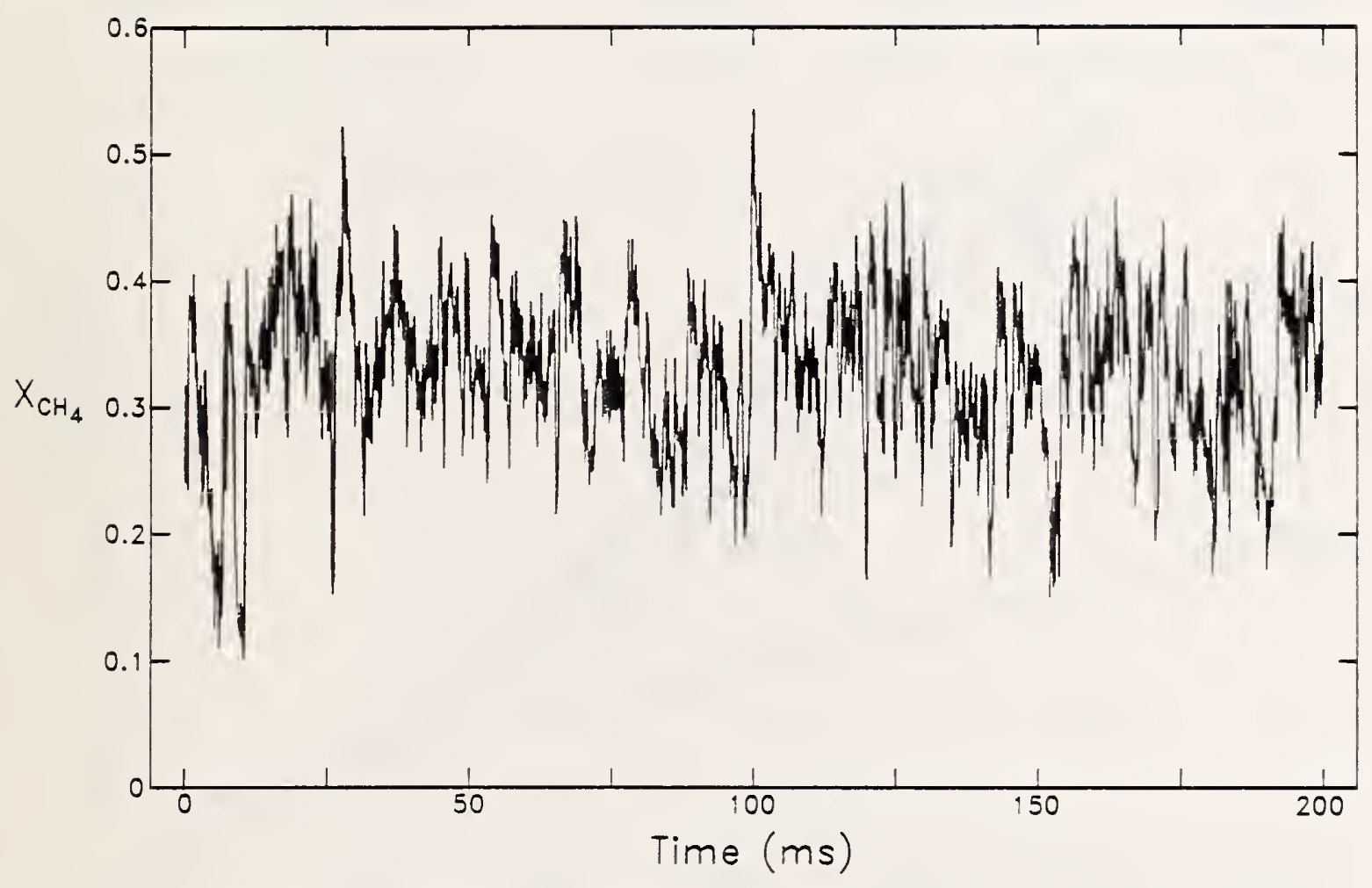

Figure 20. A portion of the time-resolved methane concentration fluctuations within a turbulent jet of methane $(\operatorname{Re}=3950)$ flowing into a slow coflow of air is shown. The measurements were made on the centerline at $z / r_{0}=31.5$. 


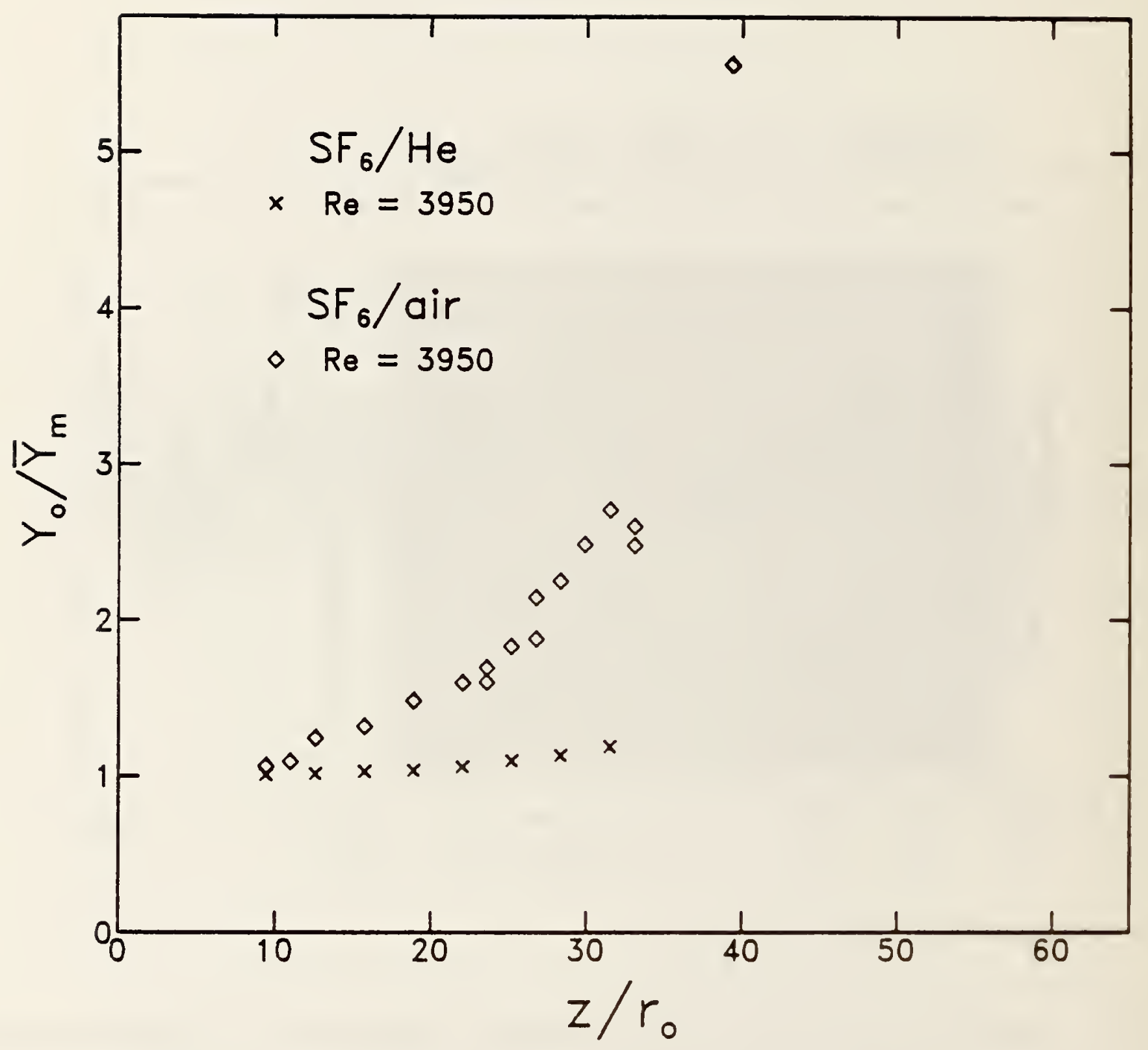

Figure 21. Values of $\mathrm{Y}_{\mathrm{o}} / \bar{Y}_{\mathrm{m}}$ as functions of $z / \mathrm{r}_{\mathrm{o}}$ are shown for the $\mathrm{SF}_{6}$ jet (Re3950) flowing into coflows of alr and helium. 


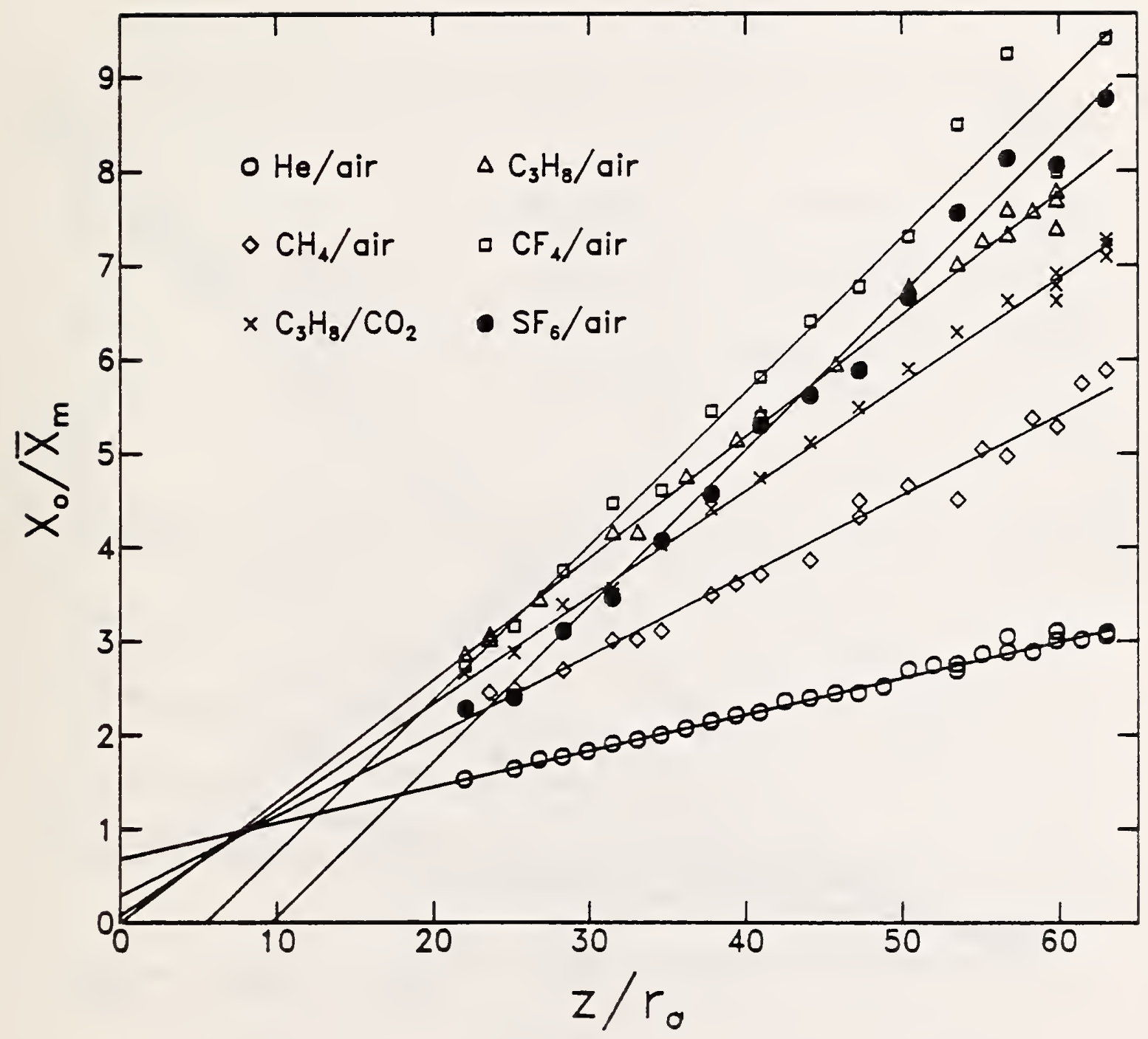

Figure 22. Inverse centerline values of jet gas mole fraction, normalized by multiplying by the jet gas mole fraction $\left(\mathrm{x}_{0}=1\right)$, are plotted as functions of nondimensionalized downstream distance for six different jet/coflow gas pair combinations. Straight lines are linear least squares curve fits of the data. 


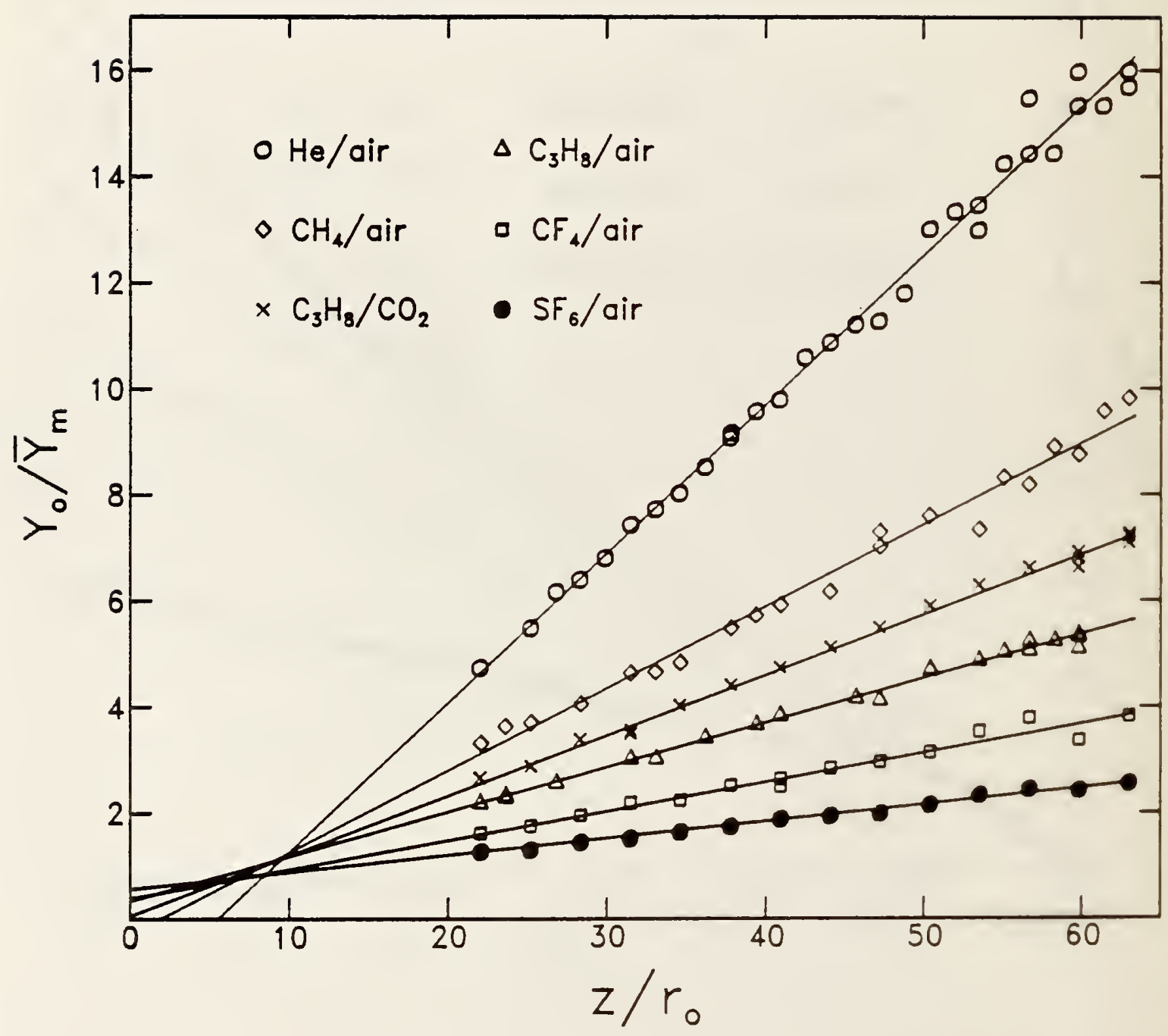

Figure 23. Values of the normalized centerline inverse mass fraction $\left(Y_{0} / \bar{Y}_{m}\right)$ are plotted as functions of $z / r_{0}$ for the six jet/coflow palrs listed. Straight lines are linear least squares curve flts of the data. 


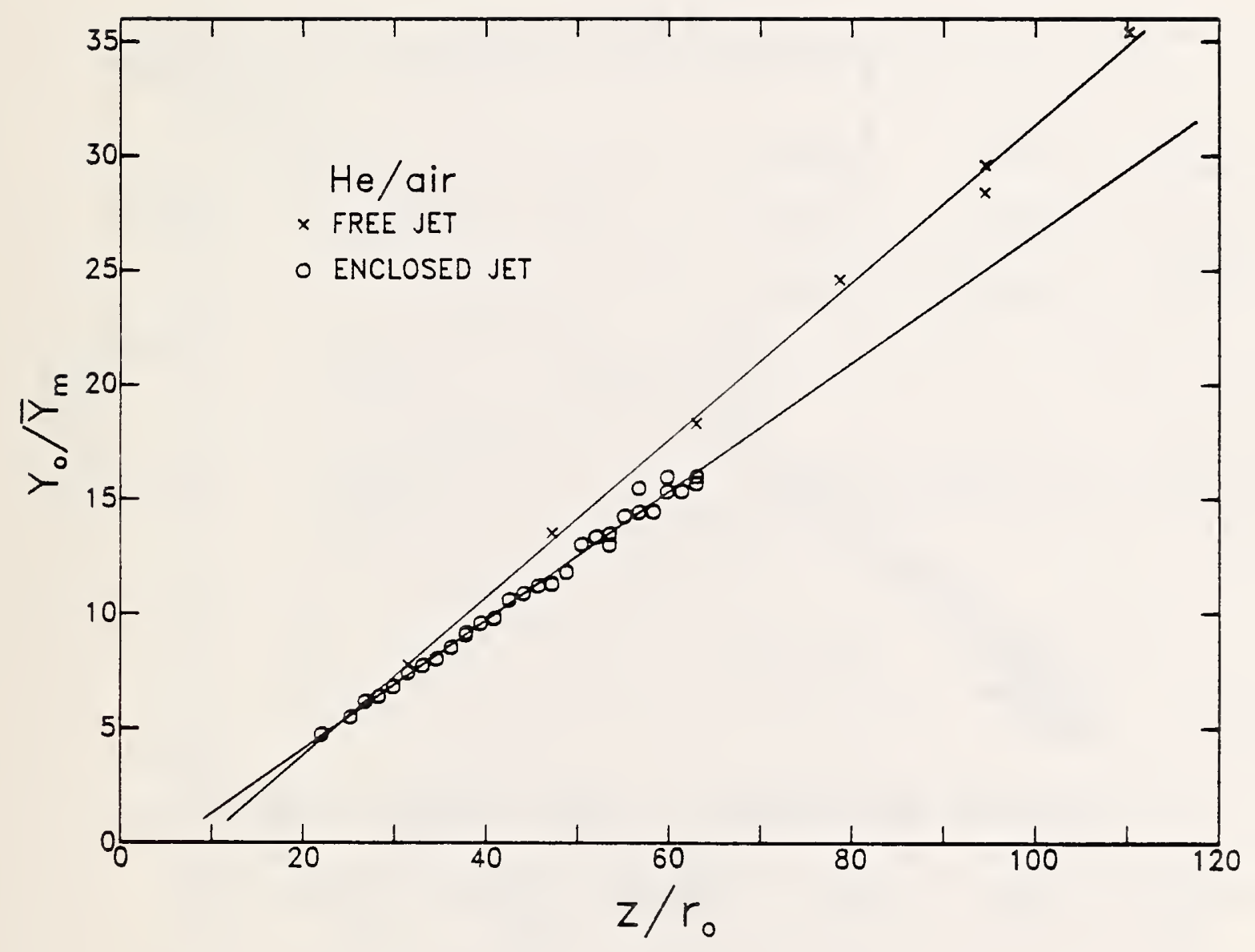

Figure 24. Values of $Y_{0} / \bar{Y}_{m}$ versus $z / r_{0}$ are plotted for the hellum fet ( $R e=$ 3950) flowing into both a slow coflow of air and the laboratory. Lines are linear least squares curve fits to the data. 


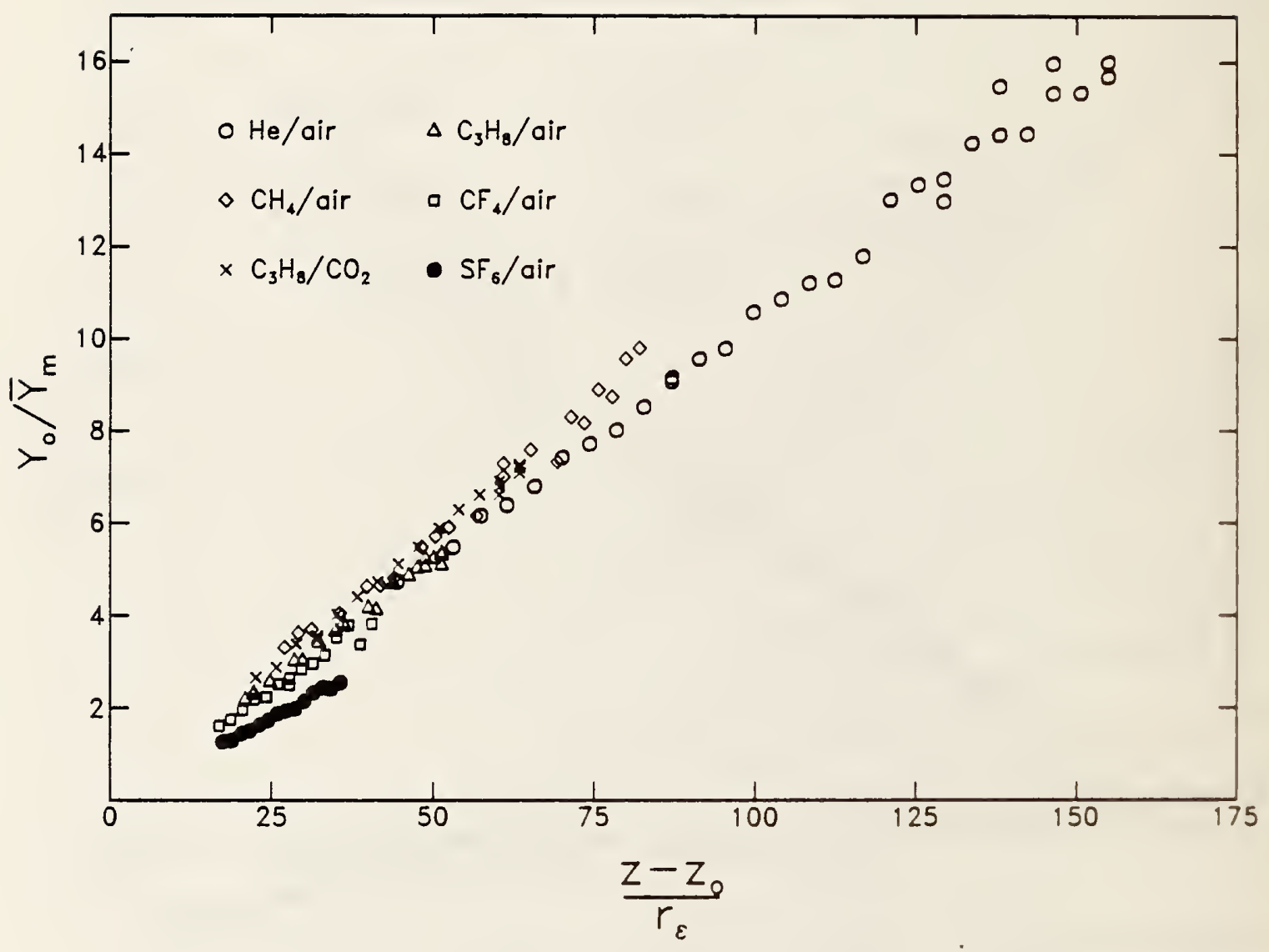

Figure 25. Experimental values of $Y_{0} / \bar{Y}_{m}$ for the six jet/coflow combinations listed are plotted as functions of downstream distance normalized by the effective radius, $r_{\varepsilon}$. Results have been offset by the appropriate values of virtual origin. Comparison with f1g. 23 indicates that the use of the effective radius concept gives a partial correlation of the data. 


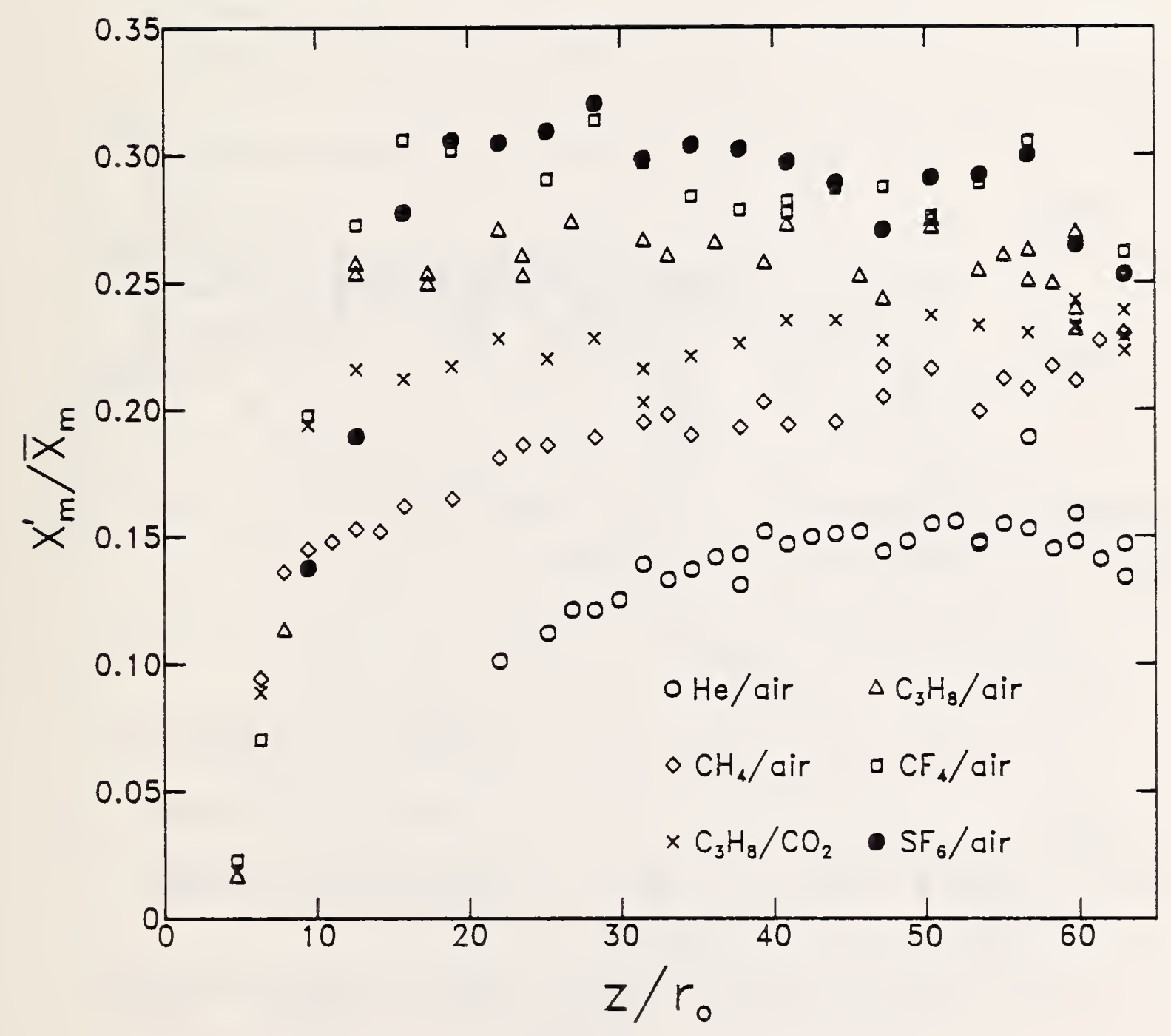

Figure 26. Experimental results of centerline unmixedness measurements in mole fraction terms $\left(\mathrm{X}_{\mathrm{m}}^{\prime} / \overline{\mathrm{X}}_{\mathrm{m}}\right)$ are plotted as functions of $\mathrm{z} / \mathrm{r}$ for the six jet/coflow pairs listed. 


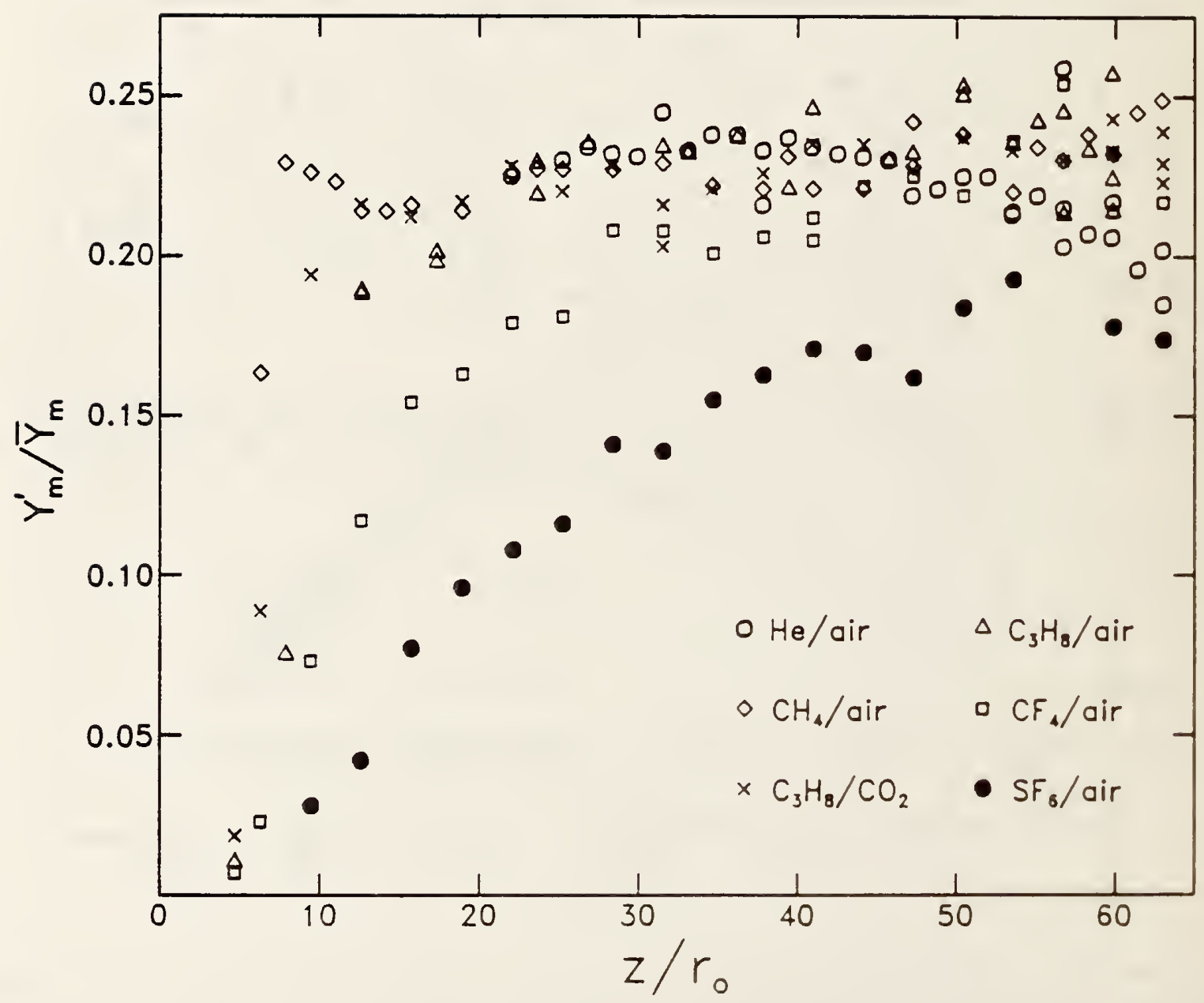

Figure 27. The results of $\mathrm{Y}_{\mathrm{m}}^{\prime} / \overline{\mathrm{Y}}_{\mathrm{m}}$ measurements for the jet/coflow pairs listed are plotted as functions of $z / r_{0}$. Note that the values for each flow approach a common asymptote of $\left(Y_{m}^{\prime} / \bar{Y}_{m}\right)_{\text {asym }} \simeq 0.23$. 


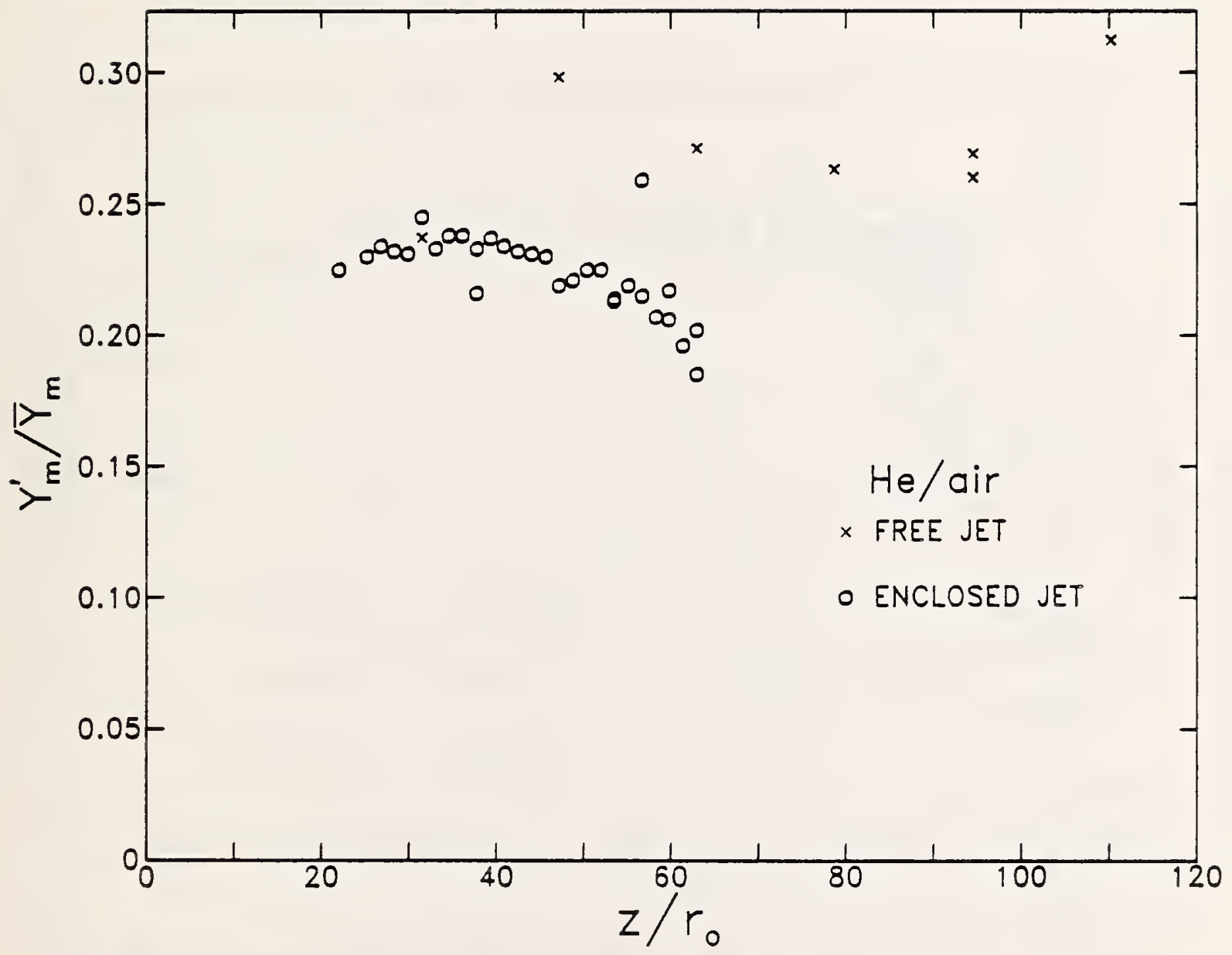

Figure 28. Values of $\mathrm{Y}_{\mathrm{m}}^{\prime} / \bar{Y}_{\mathrm{m}}$ are plotted as functions of $z / \mathrm{r}_{0}$ for the helium jet $(\operatorname{Re}=3950)$ flowing intc a coflow of air and into the laboratory (free jet). 


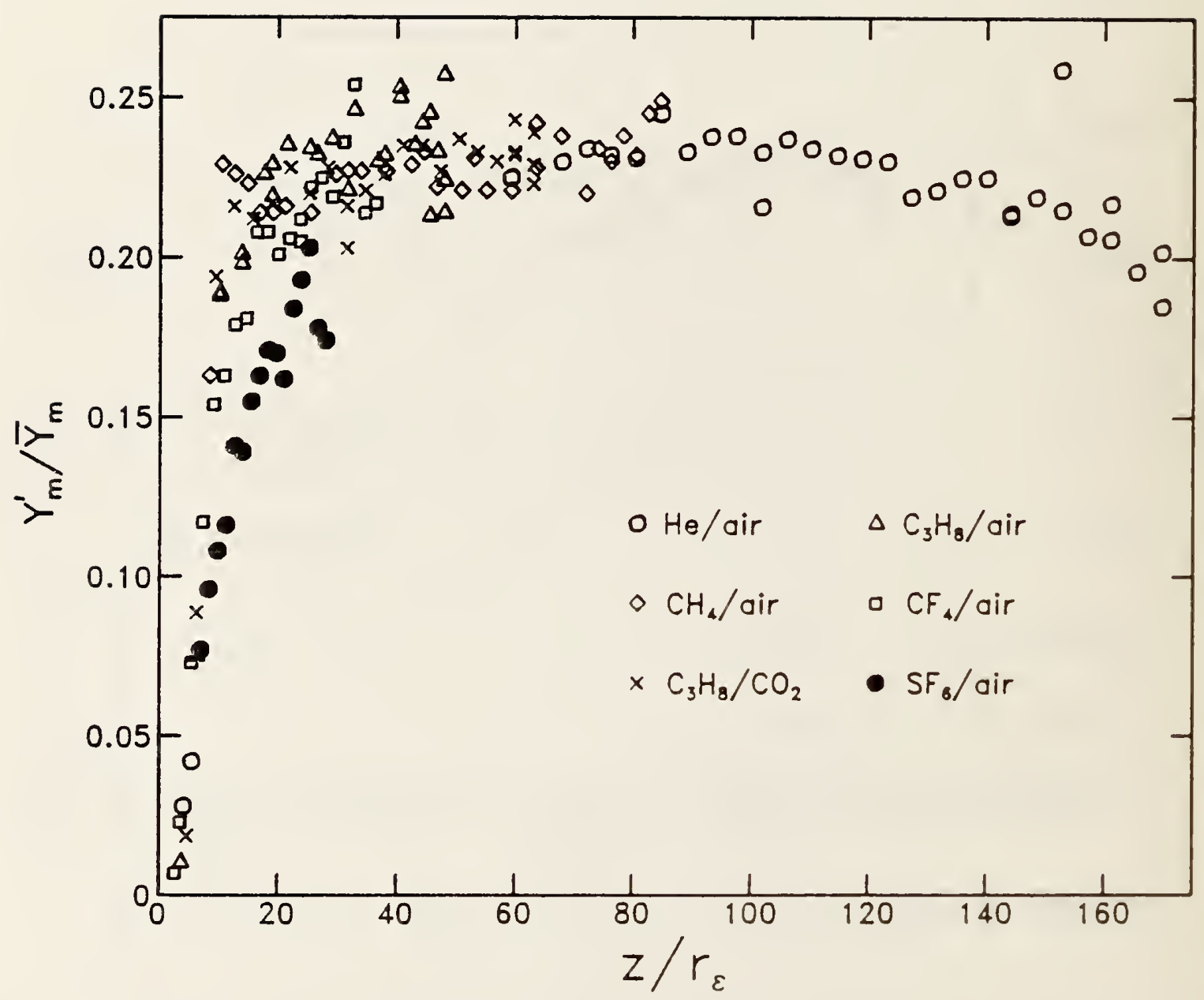

Figure 29. Mass fraction unmixedness values for the s1x jet/coflow gas comb1nations are plotted as functions of the downstream distance nond1mensionalized by dividing by the effective radıus. An excellent correlation of the data results. 


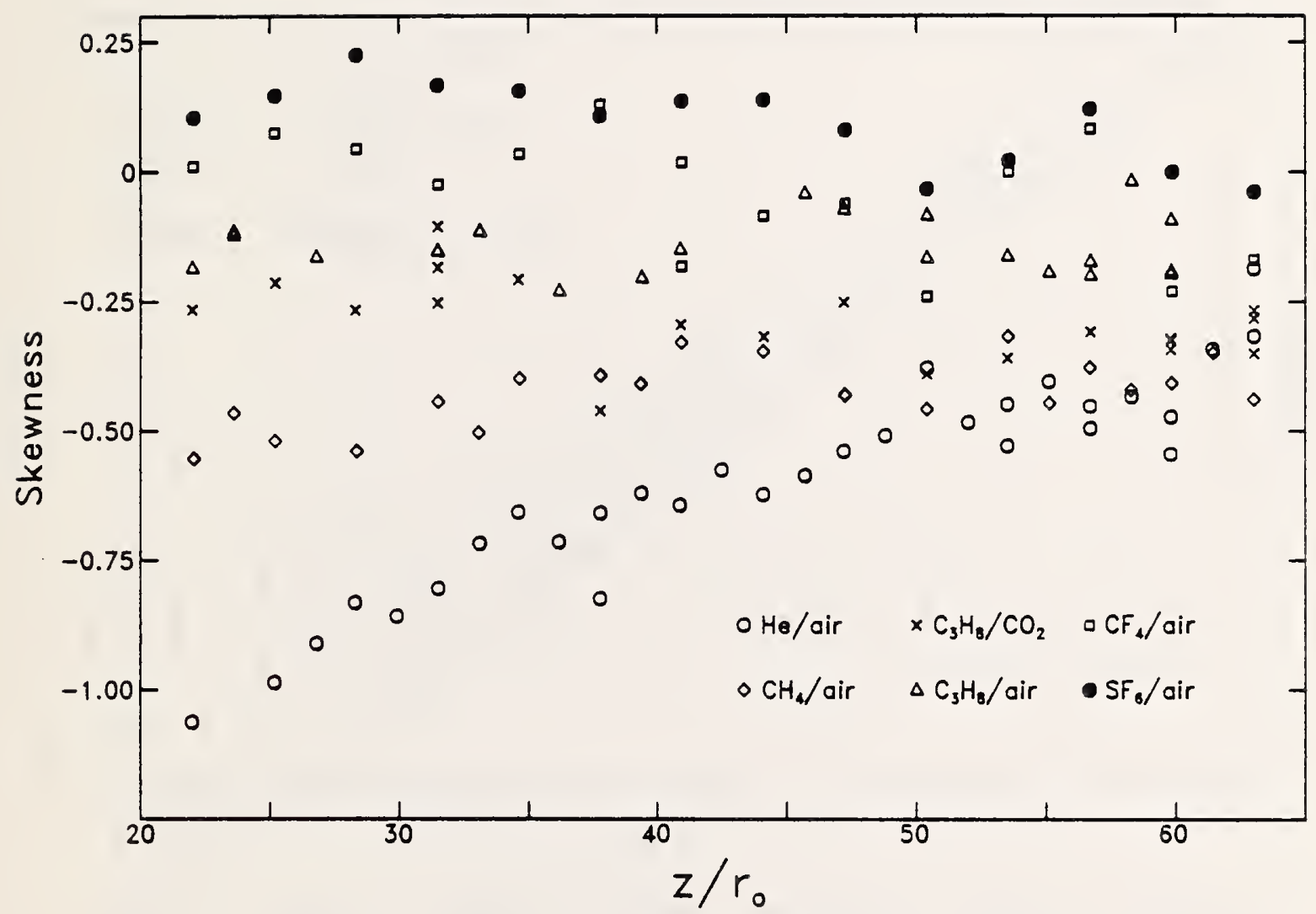

Figure 30. Skewness values for centerline concentration fluctuations are plotted as functions of $z / x_{0}$ for the jet/coflow gas pairs listed. 


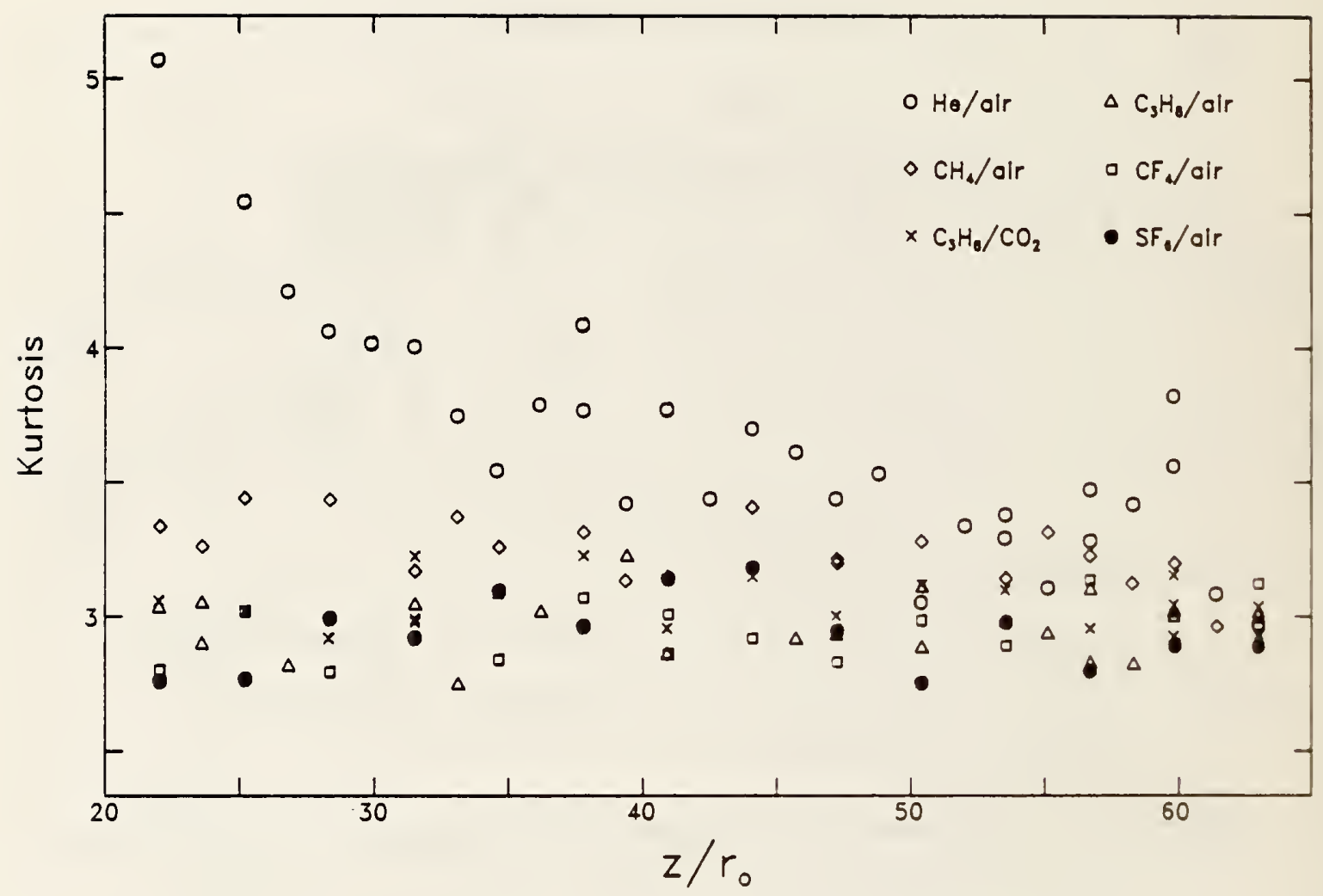

Figure 31. Kurtosis values for centerline concentration fluctuations are plotted as functions of $z / r_{0}$ for the jet/coflow gas palrs listed. 


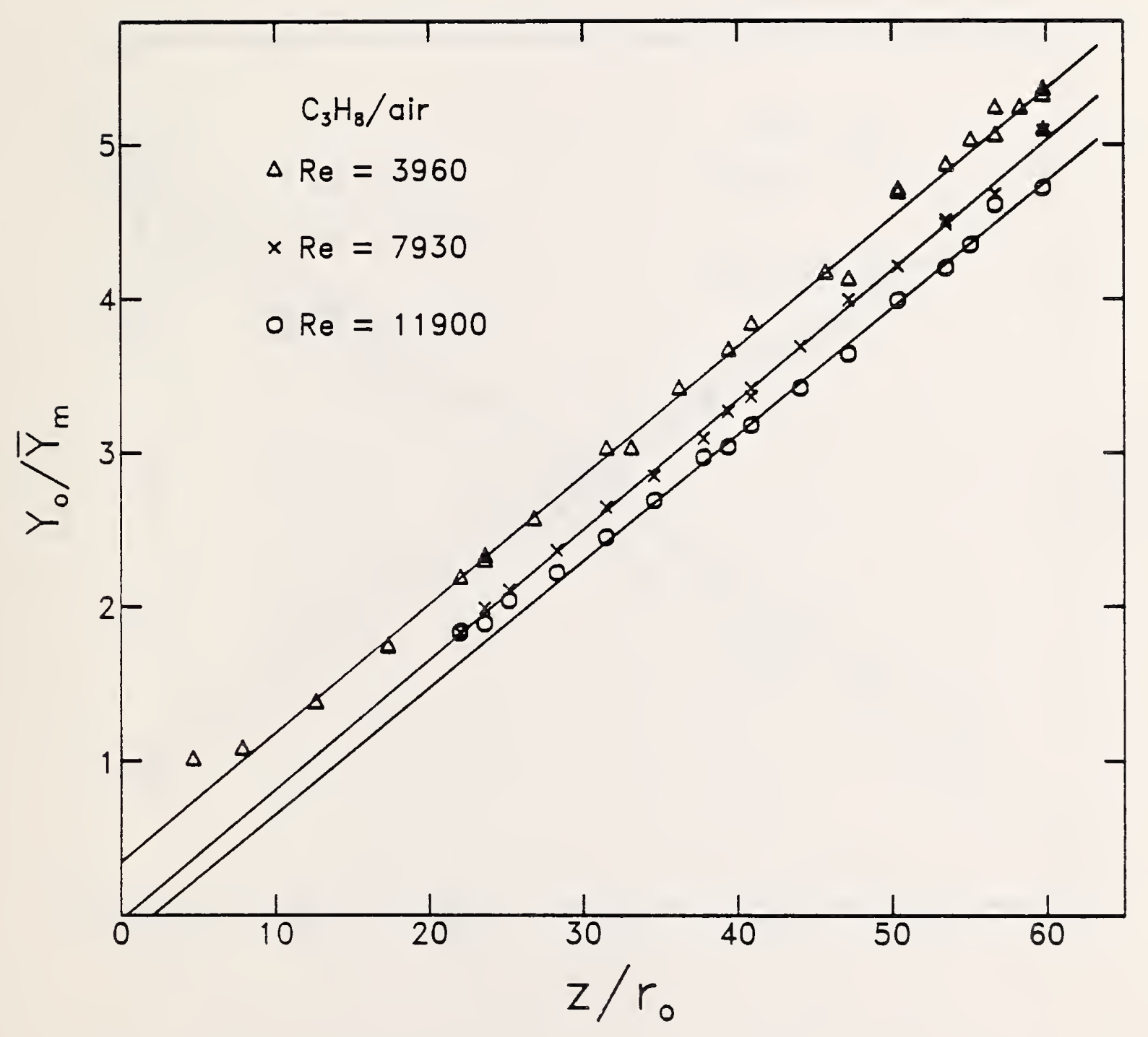

Figure 32. $Y_{0} / \vec{Y}_{m}$ values are plotted against $z / r_{0}$ for propane fets having the three different Reynolds numbers 1isted. The coflow gas is air. Straight lines represent linear least squares curve fits of the data. 


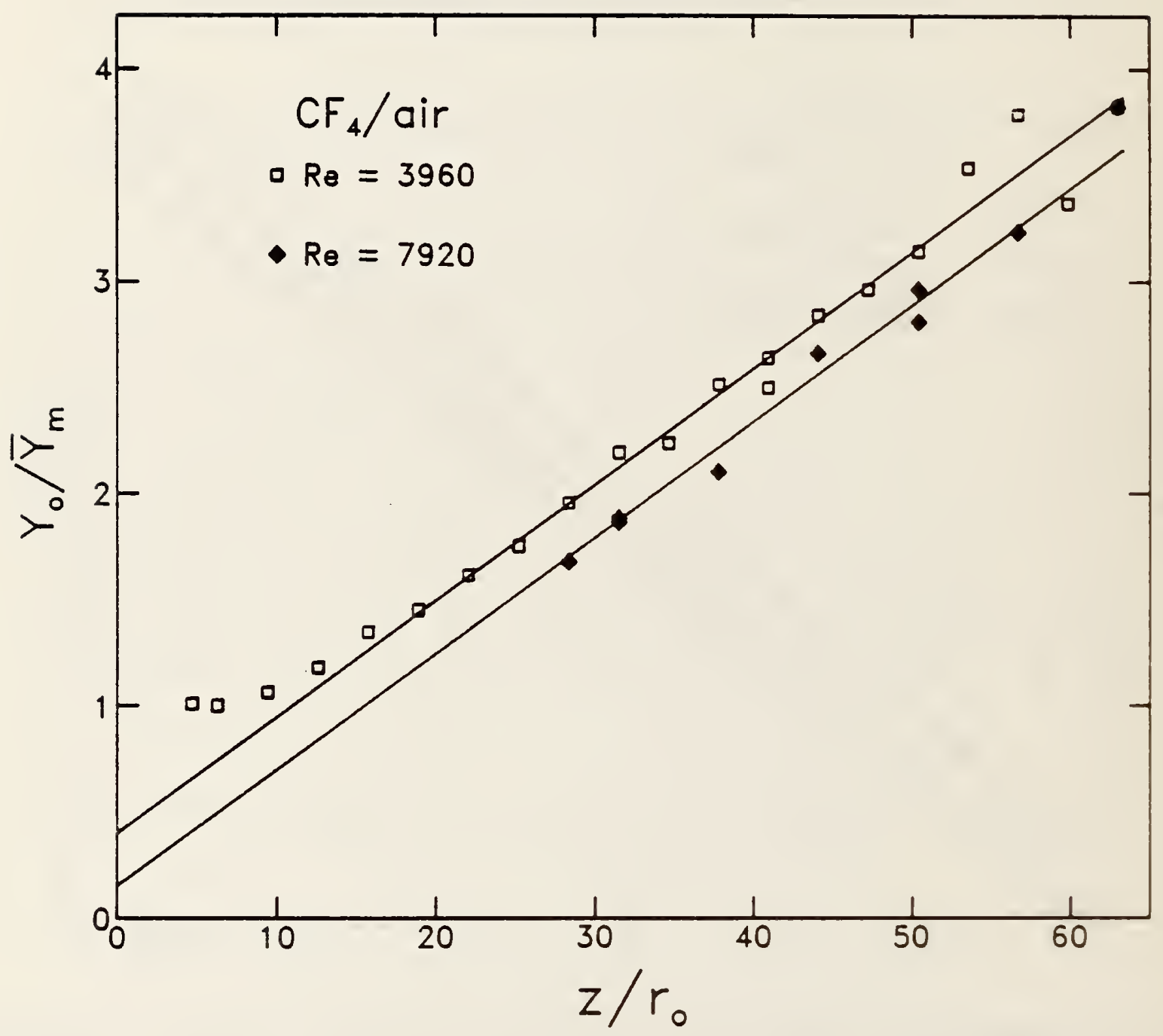

Figure 33. $Y_{o} / \bar{Y}_{m}$ values for two different Re flows of carbon tetrafluoride into air coflows are plotted as functions of $z / r_{0}$. Linear least squares curve fits to the data are represented by the lines. 


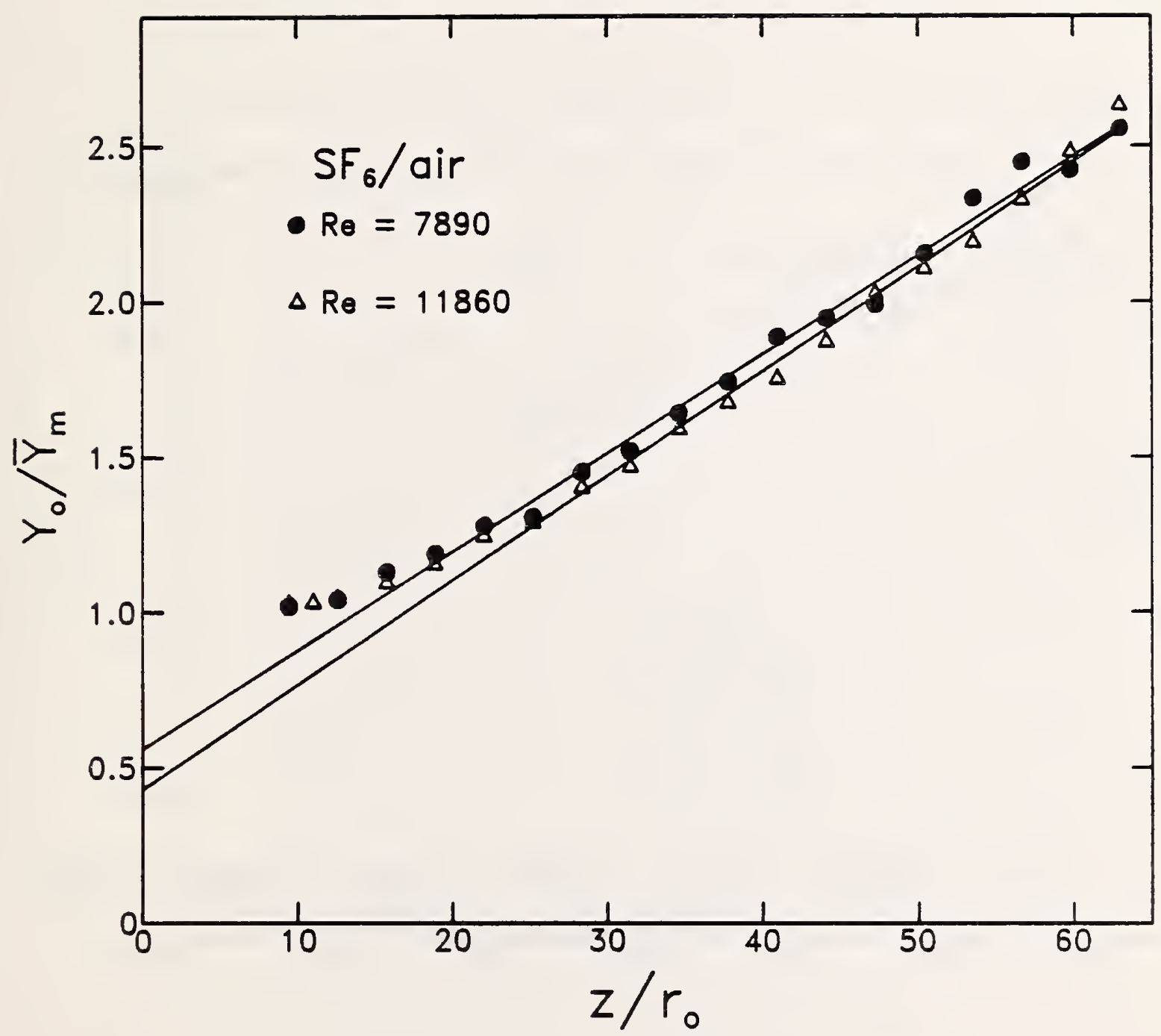

Figure 34. Values of $\mathrm{Y}_{0} / \overline{\mathrm{Y}}_{\mathrm{m}}$ for $\mathrm{SF}_{6}$ jets ar Reynolds numbers of 7890 and 11,860 are shown as functions of $z / x_{0}$. Straight lines represent the results of linear least squares curve fits of the data. 


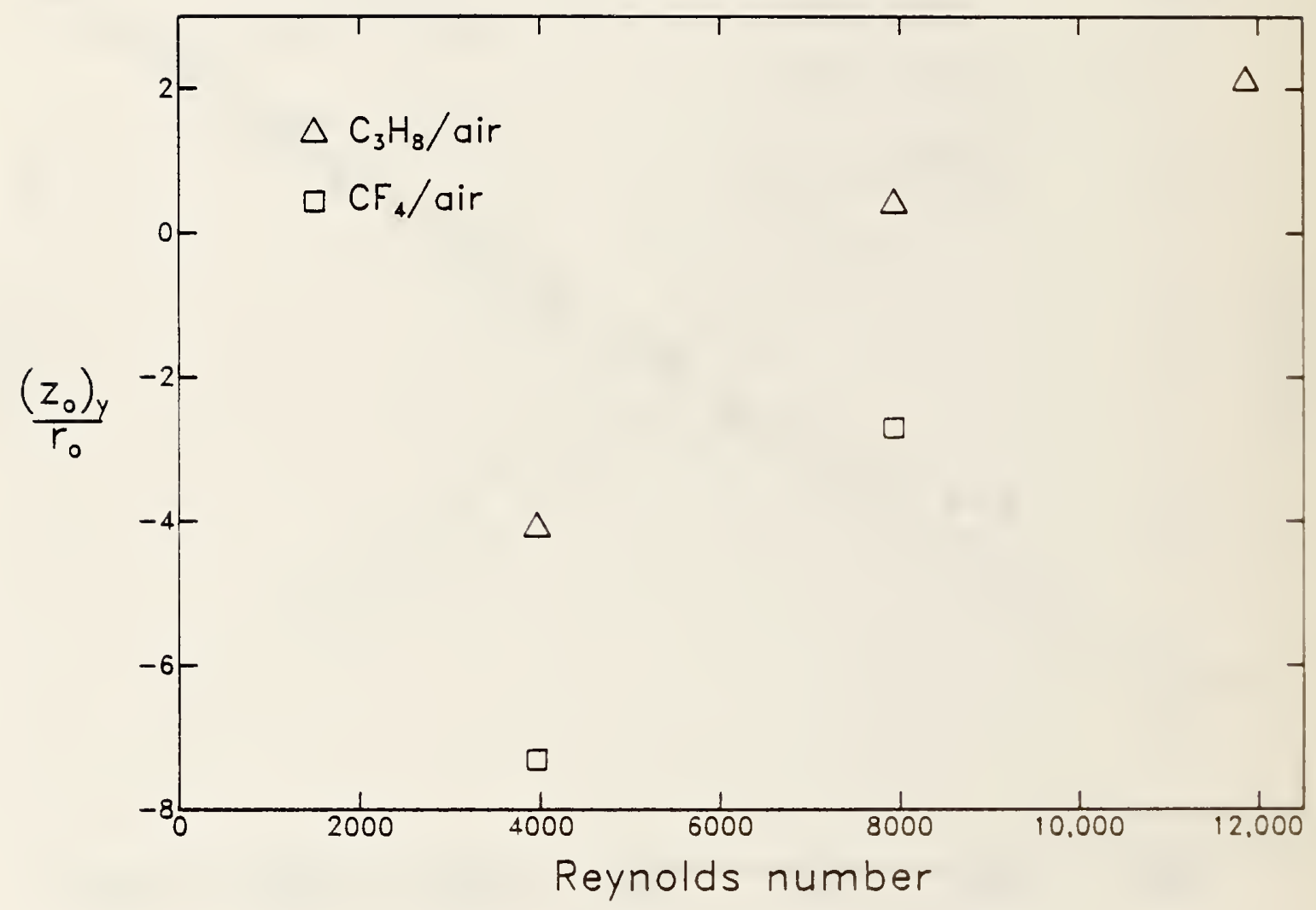

Figure 35. Virtual origins which are obtained from centerline concentration measurements in the $\mathrm{C}_{3} \mathrm{H}_{8}$ /air and $\mathrm{CF}_{4}$ /air flows are plotted as functions of jet Reynolds number. 


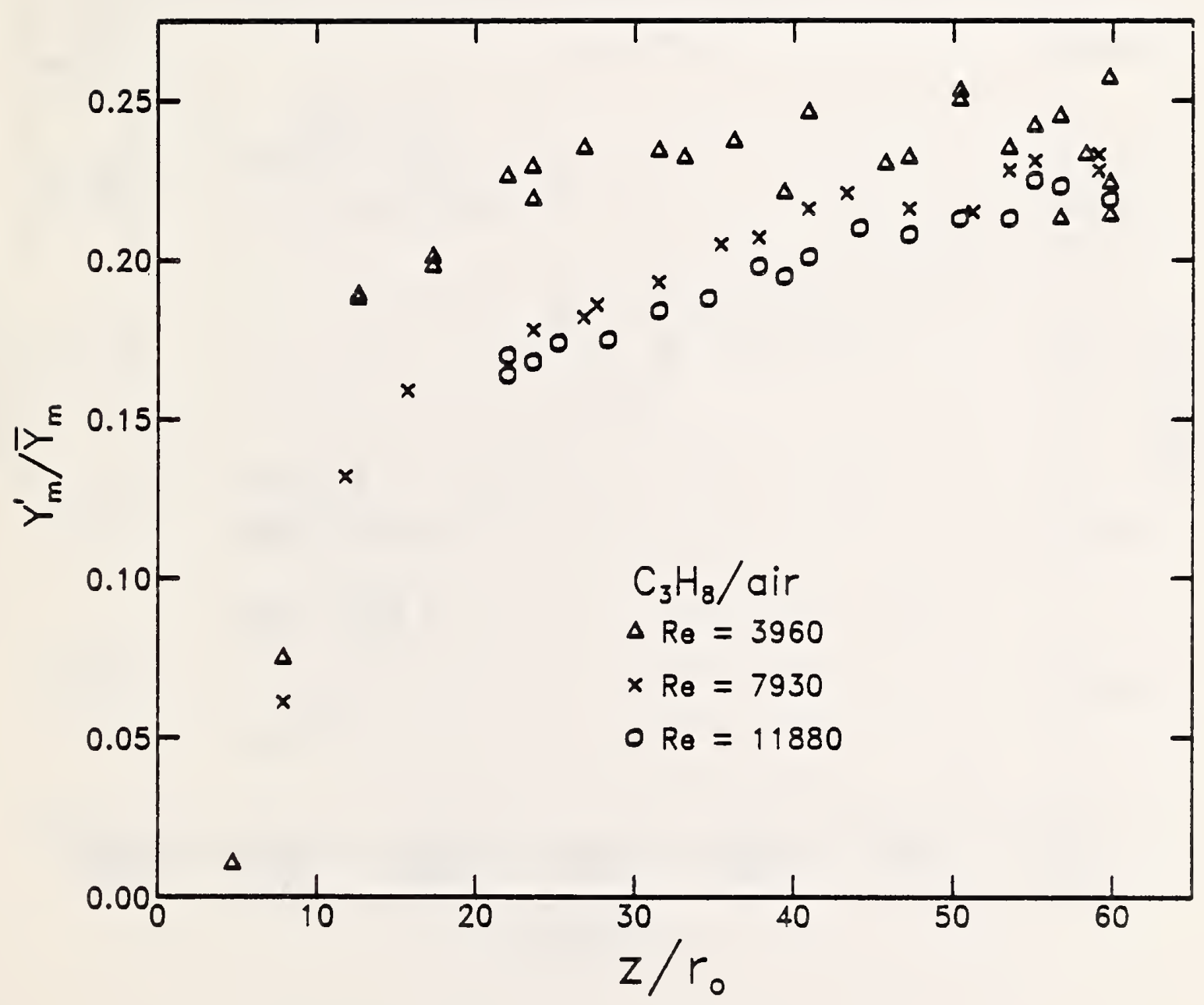

Figure 36. Mass fraction unmixedness values are plotted as functions of $z / r_{0}$ for three different Re jets of propane entering slow coflows of air. 


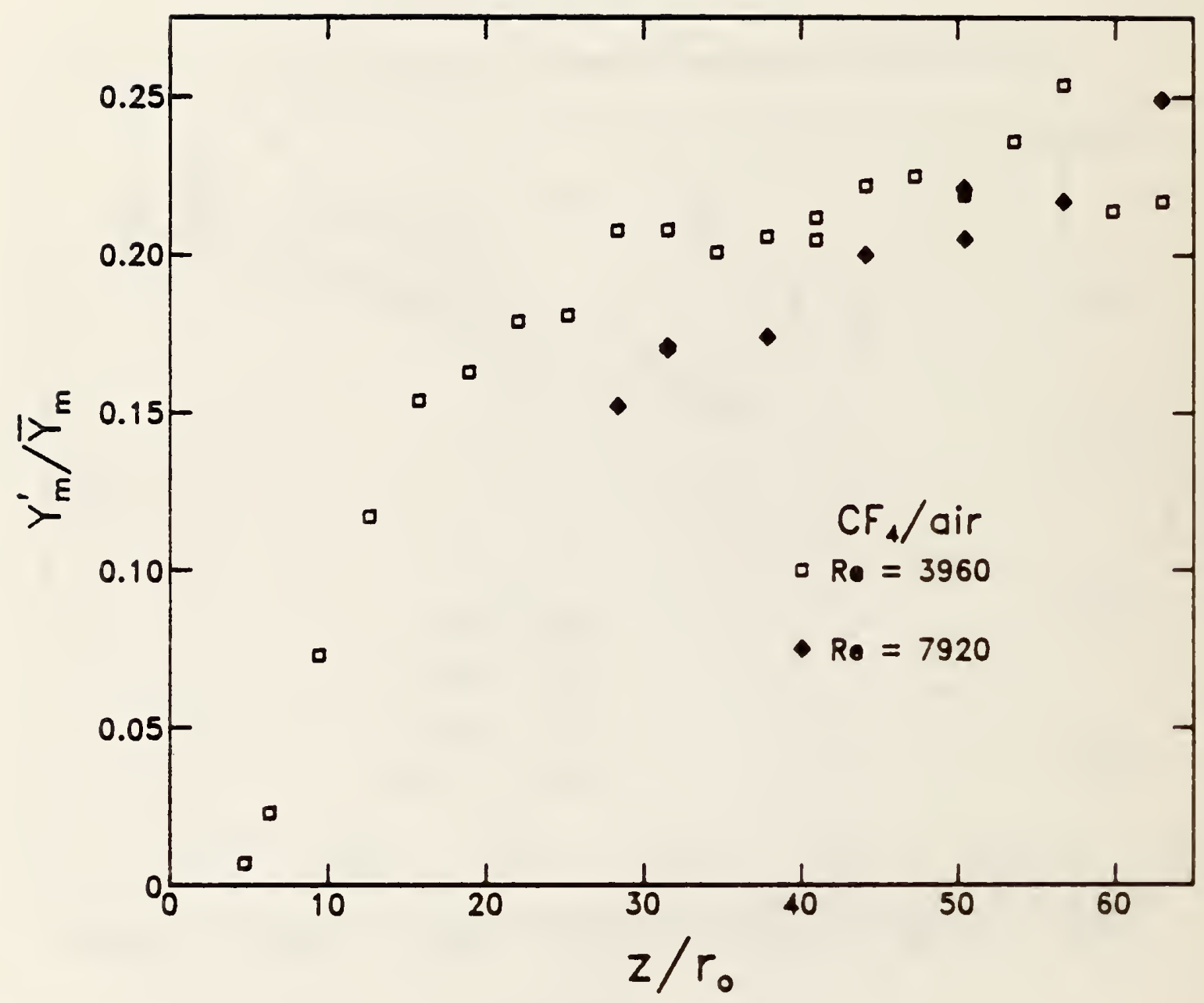

Figure 37. Mass fraction inmixedness values are plotted as functions of $z / \tau$, for two different Re jets of $\mathrm{CF}_{4}$ flowing into slow alr coflows. 


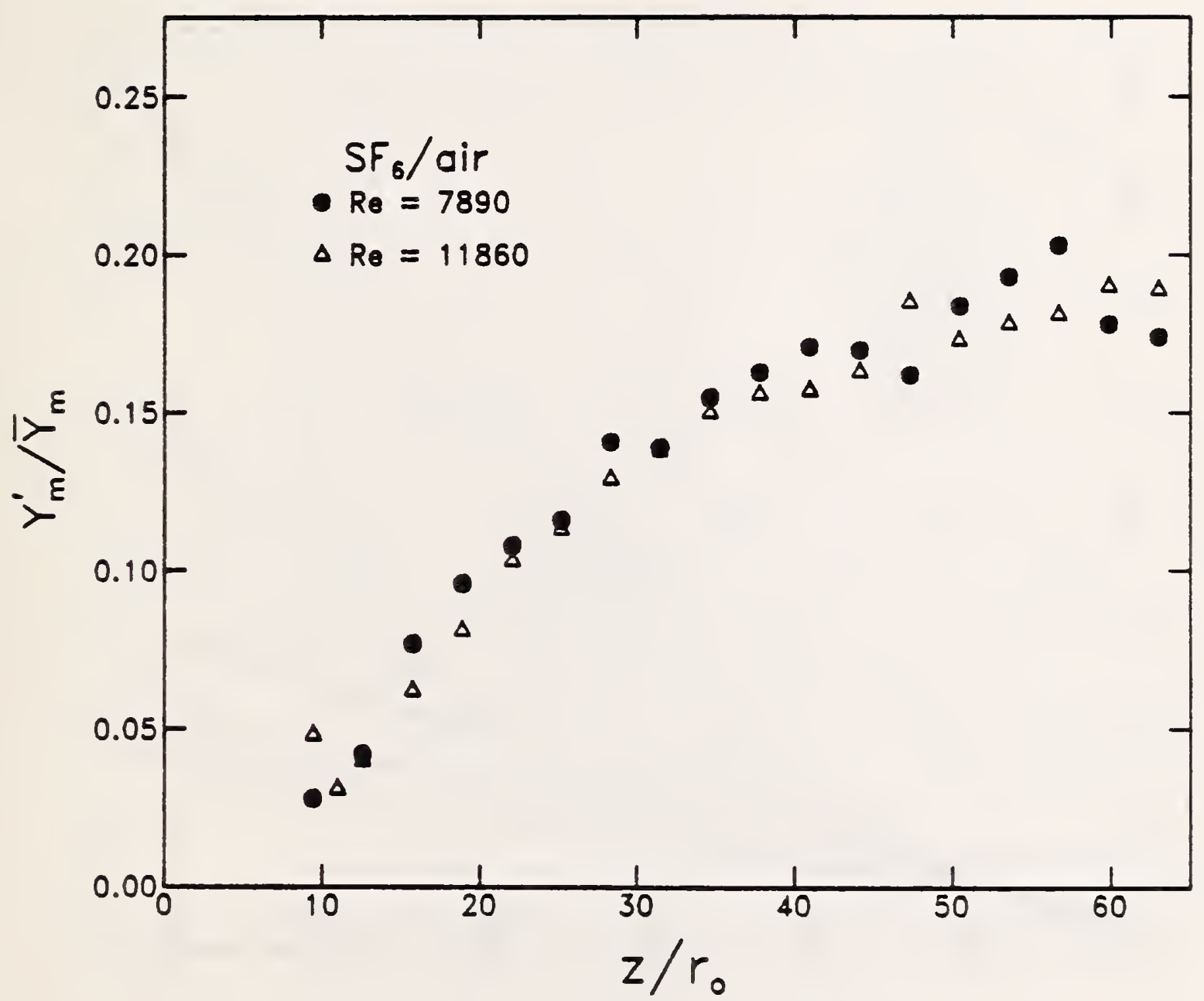

F1gure 38. Unmixedness values for jets of $\mathrm{SF}_{6}$ at Reynolds numbers of 7890 and 11,860 are plotted 28 functions of $z / r_{0}$. The coflow gas is air. 


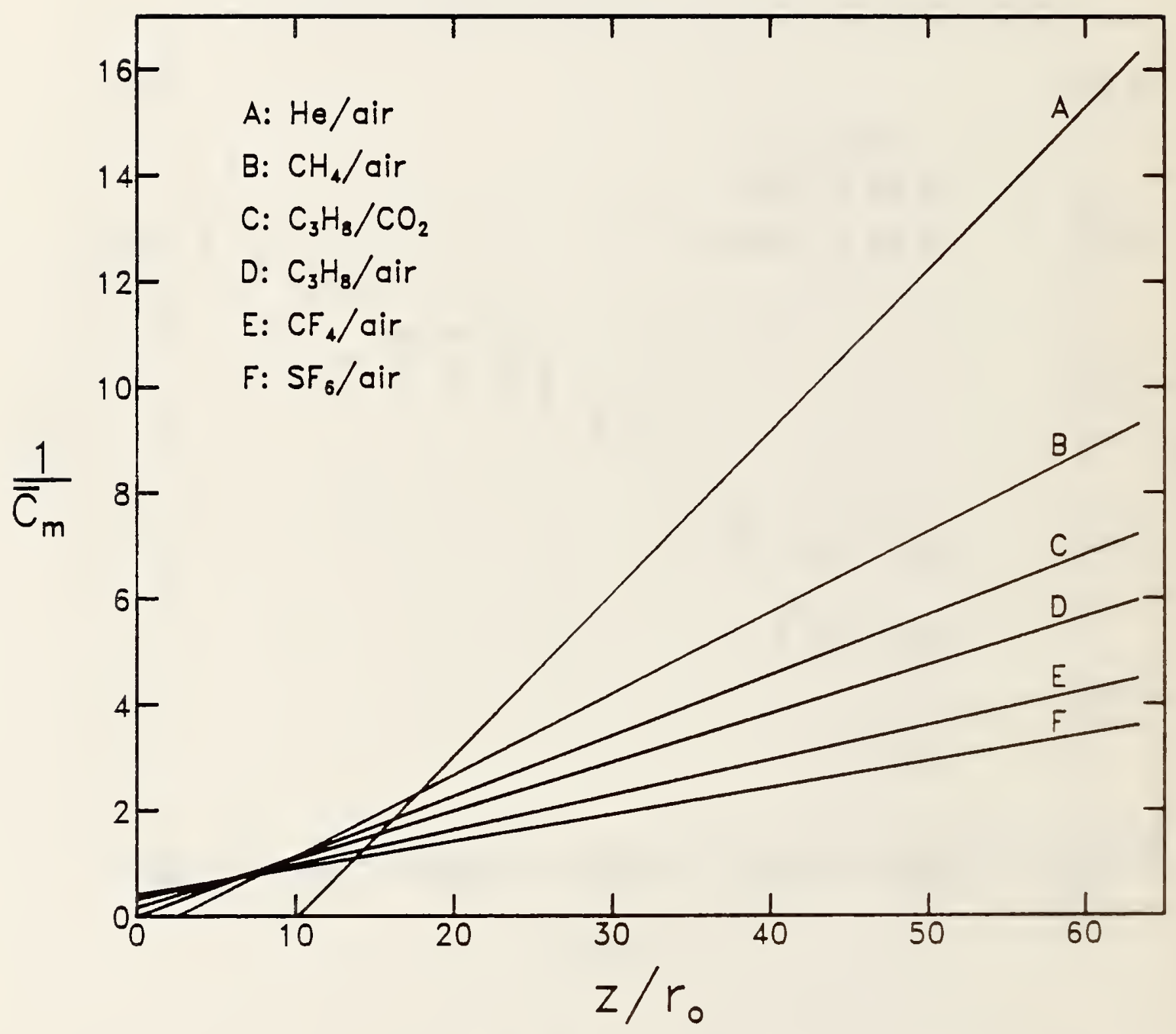

Figure 39. Values of $1 / \bar{C}_{m}$ calculated using eq. (34) are plotted as functions of $z / r_{0}$ for the six gas pairs listed. These results are based on a method suggested by Thring and Newby [18]. 


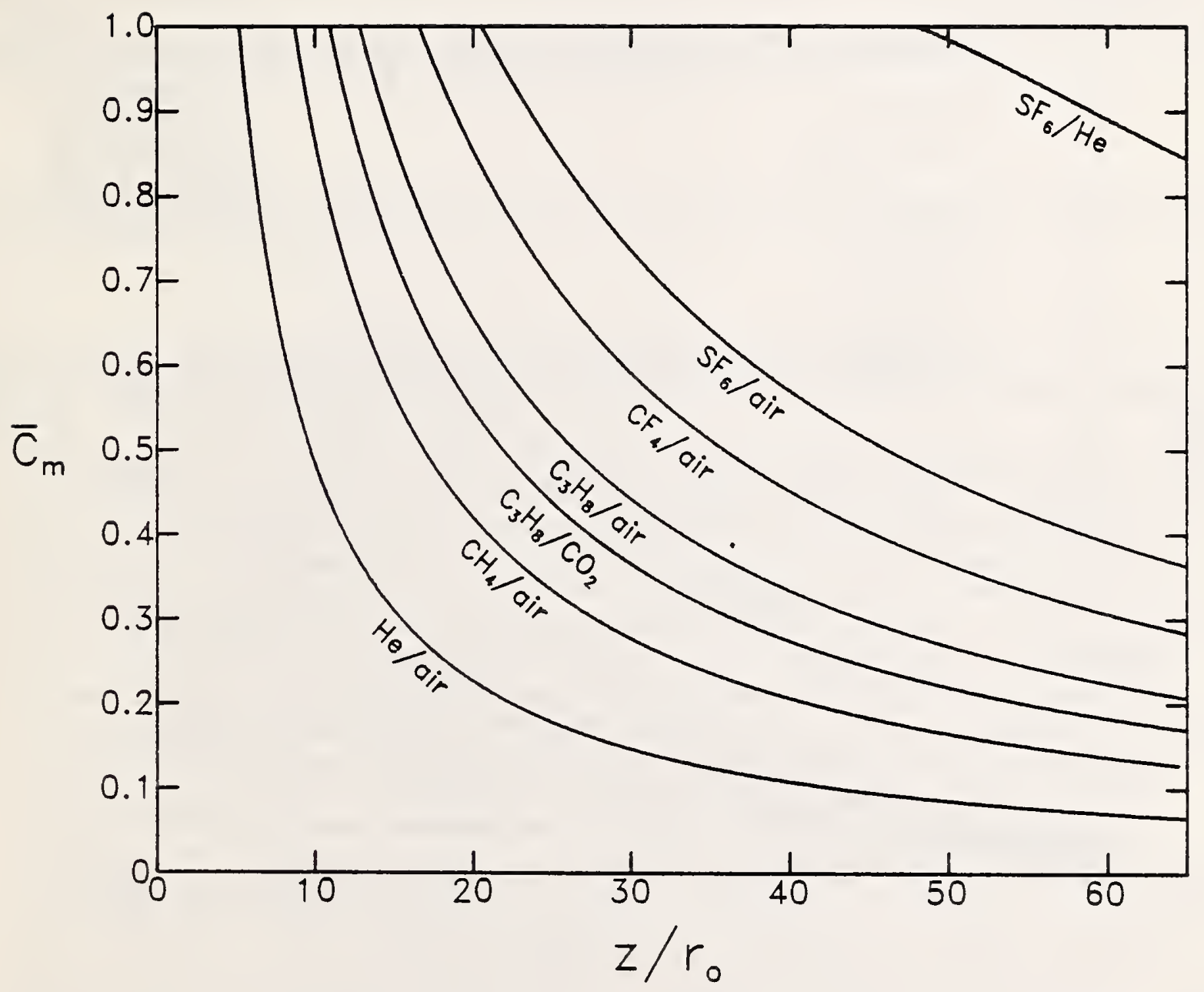

Figure 40. Values of $\bar{C}_{m}$ calculated in the manner suggested by Kodi $[5,23]$ are plotted as functions of $z / x_{0}$. Values of jet to surrounding gas density ratios correspond to those used in this study. 


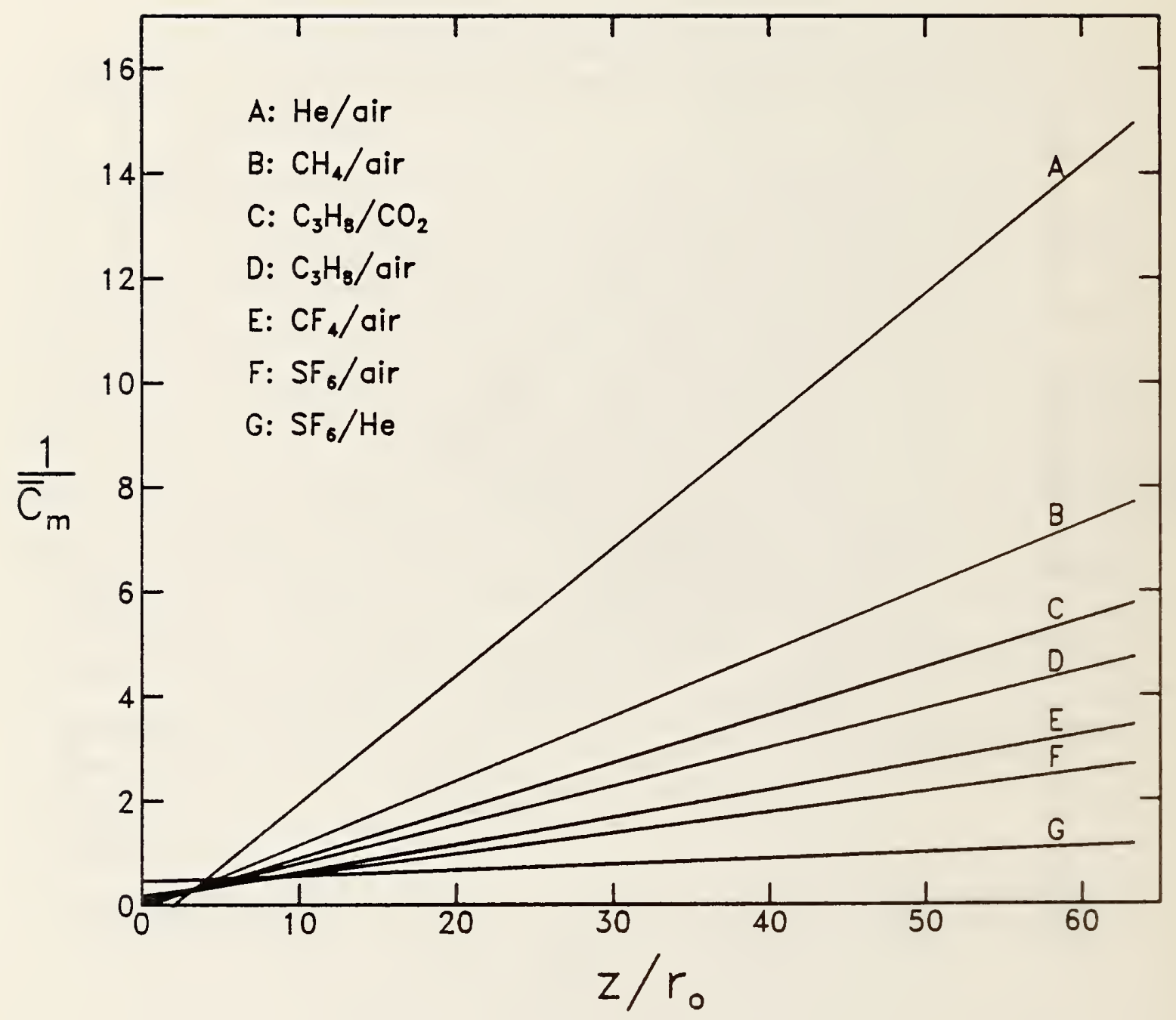

Figure 41. The calculations shown in $\mathrm{f} f \mathrm{~g} .40$ have been redrawn as $1 / \bar{C}_{\text {m }}$ versus $2 / \mathrm{r}_{0}$. Comparison of this figure with $\mathrm{fig} .39$ shows that the approximate treatments of Thring and Newby [18] and Rod1 [5,23] give similar predictions for the dependence of average centerline concentration falloff on the jet/coflow gas density ratio. 
4. TITLE AND SUBTITLE

Effects of Global Density and Reynolds Number Variations on Mixing in Turbulent, Axisymmetric Jets

5. $A \cup T H O R(S)$

William M. Pitts

6. PERFORMING ORGANIZATION (If joint or other than NBS, see instructions)

NATIONAL BUREAU OF STANDARDS

DEPARTMENT OF COMMERCE

WASHINGTON, D.C. 20234

9. SPONSORING ORGANIZATION NAME AND COMPLETE ADDRESS (Street, City. Stote, ZIP)

7. Contract/Grant No.

AFOSR-ISSA-00012

3. Type of Report \& Period Covered

Internal Report \&

Archival Publication

Air Force Office of Scientific Research

Bolling AFB

Building 410

Washington, DC 20332

10. SUPPLEMENTARY NOTES

Document describes a computer program; SF-185. FIPS Software Summary, is attached.

11. ABSTRACT (A 200-word or less factual summory of most significont information. If document includes a significant bibliogrophy or literoture survey. mention it here)

Shadowgraphy and laser-induced Rayleigh light scattering measurements of centerline concentration have been utilized to investigate the effects of global density and Reynolds number variations on the mixing behavior of a turbulent, axisymmetric jet. Density variations are introduced by using different gases for the jet and the surrounding coflow. Values of the jet/coflow density ratio have been varied from 0.14 to 37 . The shadowgraph measurements give a qualitative indication of the variations in average mixing behavior and turbulent structure which occur when the jet/coflow density ratio and the $\mathrm{Re}$ are varied. These trends are quantified by the Rayleigh scattering concentration measurements which give measurements of average centerline concentration as well as the second through fourth moments of the concentration fluctuations. Extensive comparisons of experimental results are made with previous literature findings. Integral analysis has been employed to provide. a framework for analysis of the data. The results of this study have led to the proposal of a simple, qualitative theory based on two reasonable assumptions concernting the flow behavior which gives predictions which are in agreement with the experimental findings.

12. KEY WOROS (Six to twelve entries: olphabetical order: copitalize only proper names; and seporate key words by semicolons) concentration fluctuations; concentration measurement; density effects; flow visualization; Rayleigh light scattering; Reynolds number; shadowgraphy; turbulent flow

Order From Superintendent of Documents, U.S. Government Printing Office, Washıngton, D.C. 20402.

X Order From National Technical Information Service (NTIS), Springfield, VA. 22161

14. NO. OF PRINTED PAGES

172

15. Price 


